\title{
Bright Southern Variable Stars in the bRing Survey
}

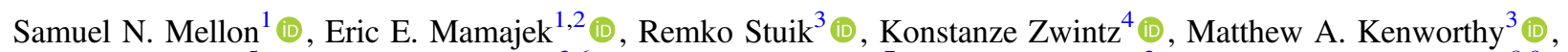

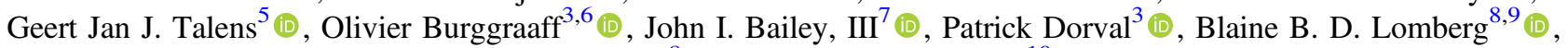 \\ Rudi B. Kuhn ${ }^{8}$ (D), and Michael J. Ireland ${ }^{10}(\mathbb{D}$ \\ ${ }^{1}$ Department of Physics \& Astronomy, University of Rochester, 500 Wilson Blvd., Rochester, NY 14627, USA; smellon@ur.rochester.edu \\ 2 Jet Propulsion Laboratory, California Institute of Technology, M/S 321-100, 4800 Oak Grove Dr, Pasadena, CA 91109, USA \\ ${ }^{3}$ Leiden Observatory, Leiden University, P.O. Box 9513, 2300 RA Leiden, The Netherlands \\ ${ }^{4}$ Institut für Astro- und Teilchenphysik, Universität Innsbruck, Technikerstrasse 25/8, A-6020 Innsbruck, Austria \\ ${ }^{5}$ Institut de Recherche sur les Exoplanètes, Département de Physique, Université de Montréal, Montréal, QC H3C 3J7, Canada \\ ${ }^{6}$ Institute of Environmental Sciences (CML), Leiden University, P.O. Box 9518, 2300 RA Leiden, The Netherlands \\ ${ }^{7}$ Department of Physics, University of California at Santa Barbara, Santa Barbara, CA 93106, USA \\ ${ }^{8}$ South African Astronomical Observatory, Observatory Rd, Observatory Cape Town, 7700 Cape Town, South Africa \\ ${ }^{9}$ Department of Astronomy, University of Cape Town, Rondebosch, 7700 Cape Town, South Africa \\ ${ }^{10}$ Research School of Astronomy and Astrophysics, Australian National University, Canberra, ACT 2611, Australia \\ Received 2019 July 15; revised 2019 July 25; accepted 2019 July 27; published 2019 September 11
}

\begin{abstract}
In addition to monitoring the bright star $\beta$ Pic during the near-transit event for its giant exoplanet, the $\beta$ Pictoris b Ring (bRing) observatories at Siding Springs Observatory, Australia and Sutherland, South Africa have monitored the brightnesses of bright stars $(V \simeq 4-8 \mathrm{mag})$ centered on the south celestial pole $\left(\delta \leqslant-30^{\circ}\right)$ for approximately two years. Here we present a comprehensive study of the bRing time-series photometry for bright southern stars monitored between 2017 June and 2019 January. Of the 16,762 stars monitored by bRing, 353 were found to be variable. Of the variable stars, $80 \%$ had previously known variability and $20 \%$ were new variables. Each of the new variables was classified, including three new eclipsing binaries (HD 77669, HD 142049, HD 155781), $26 \delta$ Scutis, 4 slowly pulsating B stars, and others. This survey also reclassified four stars based on their period of pulsation, light curve, spectral classification, and color-magnitude information. The survey data were searched for new examples of transiting circumsecondary disk systems, but no candidates were found.
\end{abstract}

Unified Astronomy Thesaurus concepts: Variable stars (1761); Multi-periodic variable stars (1079); Long period variable stars (935); Short period variable stars (1453); Eclipsing binary stars (444); Ellipsoidal variable stars (455)

\section{Introduction}

Over the past two decades, several wide-field, ground- and space-based surveys have contributed countless hours of observations in the night sky (e.g., KELT, MASCARA, NASA's Kepler and K2 space missions: Pepper et al. 2007; Borucki et al. 2010; Howell et al. 2014; Talens et al. 2017b). The primary goal of these surveys has been the discovery of exoplanets, with each having a number of significant successes (e.g., Oberst et al. 2017; Talens et al. 2017a). A secondary result from these surveys has been the discovery and characterization of variable stars (e.g., Burggraaff et al. 2018; Collins et al. 2018).

Variable stars form the cornerstone of much of the knowledge about our universe, such as asteroseismology (e.g., Zwintz et al. 2014a, 2014b), stellar gyrochronology and rotation (e.g., Hartman et al. 2010; Gallet \& Bouvier 2013; Cargile et al. 2014; Mellon et al. 2017), classical Cepheids as standard candles for distance (e.g., Groenewegen 2018), and eclipsing systems (e.g., Mellon et al. 2017; Collins et al. 2018; Moe \& Kratter 2018). In addition to the exoplanet surveys, dedicated variable star observatories and online catalogs have fueled research in these areas (e.g., ASAS, AAVSO, ASASSN, OGLE: Pojmanski 2002; Watson et al. 2006; Udalski et al. 2008; Shappee et al. 2014). Physical properties of stellar systems can be constrained from the period and amplitude of the observed variability, such as the composite sinusoidal variability observed in the $\delta$ Scuti star $\beta$ Pictoris (Mékarnia et al. 2017; Zwintz et al. 2019).
In 2017, the $\beta$ Pictoris b Ring (bRing) instruments (located in South Africa and Australia) were constructed and brought online to observe the 2017-2018 transit of the $\beta$ Pictoris b Hill sphere (Stuik et al. 2017; Kalas et al. 2019; Mellon et al. $2019 b$ ). While observing $\beta$ Pictoris, bRing captured nearly continuous photometry of $10,000+$ bright stars $(V \sim 4-8 \mathrm{mag})$ in the southern sky $\left(\delta \leqslant-30^{\circ}\right)$. In addition to the study of the $\beta$ Pictoris $b$ Hill sphere, the bRing survey has contributed to the discovery of $\delta$ Scuti pulsations in the A1V star HD 156623 (Mellon et al. 2019a), the study of $\beta$ Pictoris' $\delta$ Scuti pulsations (Zwintz et al. 2019), and the discovery of the retrograde hot Jupiter MASCARA-4 b/bRing-1 b (Dorval et al. 2019).

In this work, we took a similar approach to the MASCARA survey of the northern sky (Burggraaff et al. 2018) and searched for periodic variations in the bRing data. This survey was also sensitive to evidence of transiting circumplanetary systems like "J1407" (V1400 Cen; Mamajek et al. 2012), or other circumsecondary disks; however, none were found. Section 2 of this work describes the data from both the South African bRing (bRing-SA) and Australian bRing (bRing-AU) stations. Section 3 details the analysis used to identify and characterize both the regular and irregular variables in the data. Section 4 provides tables and discussions of each type of variable found in cross-correlation with the VSX catalog (Watson et al. 2006) and others.

\section{Data}

The data in this work were collected between 2017 June and 2019 January by the bRing-SA and bRing-AU stations. Each 
station had two stationary cameras; one camera faced southeast $\left(\mathrm{Az}=150^{\circ} ; \mathrm{SAE}\right.$ and $\left.\mathrm{AUE}\right)$ and the other southwest $\left(\mathrm{Az}=230^{\circ}\right.$; SAW and AUW). Each camera had an FLI $4008 \times 2672$ pixel $\mathrm{CCD}$ and $f=1.4 \mathrm{~mm}$ Canon wide-angle lens, which resulted in a total field of view of $74^{\circ} \times 53^{\circ}$ with a pointing optimized for $\beta$ Pictoris $\left(\delta \simeq-53^{\circ}\right)$. Exposure times were alternated between 6.4 and $2.54 \mathrm{~s}$; these were subsequently coadded and binned to 5 minute samplings and saved to disk (Stuik et al. 2017; Talens et al. 2018).

Due to bRing's large pixel size $\left(\sim 1 \operatorname{arcmin}^{2}\right)$, blending was a significant issue for bRing. Blending was evaluated by comparing the relative brightnesses of stars located within the same bRing inner aperture (radius $=2.5$ ) as the target star (nearby stars evaluated with the ASCC catalog; Kharchenko 2001). For stars with previously known variability, blending was ignored if the original period was detected, but considered if a second period was detected or dominated the expected period. If a second period dominated the expected period in a blended star, the star was reanalyzed at the original expected period. If a star showed signs of variability in our light curves and had been previously unidentified as a variable in other surveys, blending was required to be 0 to be considered a detection. Ultimately, 16,762 stars were analyzed for this work. The stars listed in this work as new variables had no evidence of blending in their light curves.

On average, a star had observations spanning over 300 days (each star ideally received $21 \mathrm{hr}$ of continuous coverage per day); the average star had $\sim 20,000$ five minute binned data points over the entire observing window combined from all four cameras. More information on the bRing observing strategy and data calibration can be found in Stuik et al. (2017) and Talens et al. (2018). In conjunction with this work, the camera .FITS files for each star (as described in Stuik et al. 2017) were published in a Zenodo repository at doi:10.5281/ zenodo.3341783.

In interpreting the nature of the variability, $B V$ photometry was drawn from the ASCC-2.5 catalog (Kharchenko 2001) and spectral types were drawn from the literature, with most types taken from the Michigan Spectral Survey of classifications from objective-prism plates (Houk \& Cowley 1975; Houk 1978, 1982). Houk et al. (1997) has shown that for $V<8$ mag stars classified as dwarf luminosity class in the Michigan Spectral Survey, for a given 2D spectral type the intrinsic color spread rms in $B-V$ is $\sim 0.03-0.04 \mathrm{mag}$ and the intrinsic spread in absolute $V$ magnitudes is $\sim 0.4-0.5 \mathrm{mag}$, with distributions suggesting negligible contamination by more evolved giants and supergiants. The Michigan classifications for the variable stars have quality flags of $1(61.6 \%), 2(28.9 \%), 3(7.0 \%)$, and $4(0.6 \%)$, with the $93 \%$ flagged as quality 1 and 2 considered the "higher-quality" classifications (Houk 1978; Houk et al. 1997).

\section{Analysis}

The 5 minute binned data points from bRing were automatically calibrated and detrended for temporal and spatial effects from the observations (e.g., clouds, intra-pixel variations; Stuik et al. 2017; Talens et al. 2018). Using an internal custom pipeline detailed in Mellon et al. (2019a), these data were downloaded from the bRing server and further detrended for sidereal and lunar systematics as well as astrometric and color systematics. This routine also includes a barycentric correction. In addition to the detrending from previous works, we attempted to preserve the ansatz period prior to detrending by including an additional step adopted from Burggraaff et al. (2018). The data for each star from each of the four bRing cameras were treated individually and then median-combined after detrending.

\subsection{Identifying the Ansatz Period}

The time-series photometry data were analyzed using the reduction pipeline previously used and described in Mellon et al. (2019a), with a modification based on the study by Burggraaff et al. (2018). The step adopted from Burggraaff et al. (2018) to improve upon the process from Mellon et al. (2019a) was the initial identification and removal of an ansatz period from the data prior to detrending. The goal of this step was to preserve any real and significant periods from being affected by the detrending process. To find the ansatz period, a normalized Lomb-Scargle periodogram (Scargle 1982; Press et al. 1992) was generated using the ASTROPY (The Astropy Collaboration et al. 2018) library. Next, a Python routine was written using tools available in the SCIPY (Jones et al. 2001), NUMPY (Stéfan van der Walt \& Varoquaux 2011), and ASTROPY packages to identify the strongest periods in the periodogram. These periods were then compared to the wellstudied sidereal and lunar systematics present in the bRing data (the origins of these systematics and methods for removing them are thoroughly discussed in Stuik et al. 2017; Burggraaff et al. 2018; Talens et al. 2018; Mellon et al. 2019a). The strongest period that was not within $5 \%$ of one of these systematics (or the corresponding harmonics and aliases to order 5) was accepted as the ansatz period, fit with a sine, and removed from the light curve. This information was stored and was added back in after detrending.

\subsection{Detrending and Measurement of Variable Star Parameters}

The detrending routine used after the removal of ansatz period is described in Mellon et al. (2019a) and is summarized in this work. First, an astrometric correction was applied to remove data points that deviated $>3 \sigma$ from the mean path of the star on the CCD. Then, the time series was adjusted to the barycentric reference frame and a second-order CCD color correction was applied to the data. The best ansatz signal was then determined and temporarily removed from the data. Next, a median-binning routine was used to significantly reduce the strength of the lunar and sidereal systematic signals. After detrending, the ansatz signal was added back into the light curve and a composite light curve was generated from the four camera light curves using a median alignment. A new periodogram was calculated from this composite light curve. Finally, a plot of the composite light curve, a periodogram, and a phase-folded light curve on the most likely variability period was generated for analysis. These plots were used to identify variables in the data by eye. An example is plotted in Figure 1 for the $\delta$ Scuti HD 156623. The plots generated for this work were included in the same Zenodo repository, doi: 10.5281/ zenodo.3341783, as the data.

The measurements and information used to construct the tables of variable stars (see Section 4) were also generated. The best periods for variables were taken directly from the composite light-curve periodograms and verified by comparing measurements from the four independent camera data sets. A previously unseen frequency was accepted if it was detected in 
HD 156623
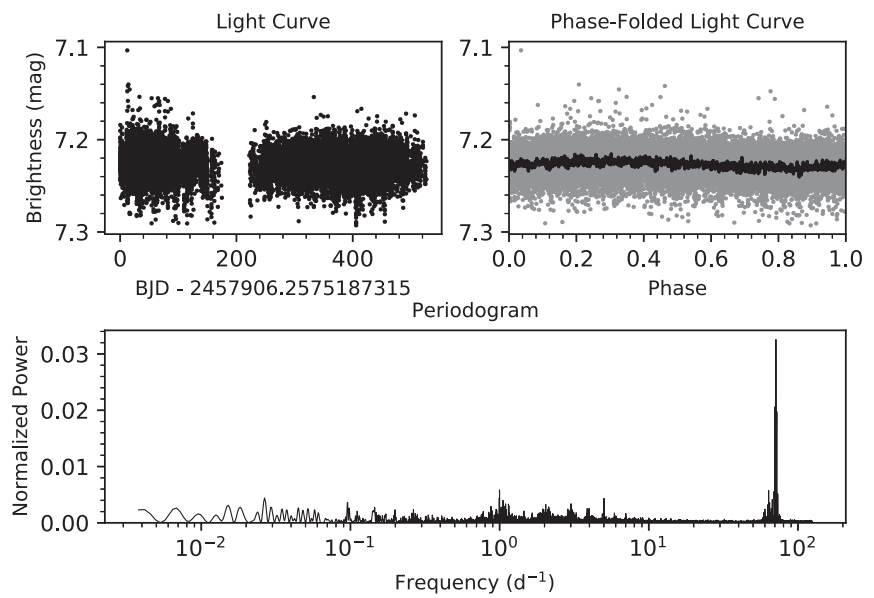

Figure 1. Example plot of the $\delta$ Scuti HD 156623. The top left panel contains the light curve of the star. The top right panel contains the light curve of the star (gray dots) phase-folded on the primary period with a running median fit (solid curve). The bottom panel contains the normalized LS periodogram.

at least one camera from each site. Uncertainties for the frequencies were measured from the standard deviation in the detected frequencies; amplitude uncertainties were calculated using methods from Montgomery \& Odonoghue (1999). We compared the Montgomery \& Odonoghue (1999) frequency uncertainty measurements to the measurements from using the four camera data sets and found the uncertainties were typically underestimated by a factor of $\sim 5$. This is expected, as the uncertainties from Montgomery \& Odonoghue (1999) were noted in their work as lower limits on the errors in these measurements.

\subsection{False Positives}

The strongest periodogram frequencies from the stars in this study were used to identify remaining low-frequency $\left(f<1.5 \mathrm{day}^{-1}\right)$ and high-frequency $\left(f>1.5 \mathrm{day}^{-1}\right)$ false positives due to systematics in the bRing system. To do this, a density plot of the strongest frequencies was generated with bin sizes of $0.01 \mathrm{day}^{-1}$ (Figure 2). The left panel focuses on the low-frequency false positives, which have been discussed thoroughly in Stuik et al. (2017), Talens et al. (2018), Burggraaff et al. (2018), Mellon et al. (2019a). The highfrequency systematics were observed to be more numerous and scattered, but are weaker by a factor of $\sim 10$ compared to the low-frequency systematics and are roughly a factor of 10 above the noise floor $\left(\simeq 10^{-4}\right)$ of the plot. Possible sources include the 288 day $^{-1}$ (5 minute) sampling frequency of bRing and its beats/aliases and electromagnetic interference within bRing. The bRing detrending routines are continuing to be internally developed to minimize the effects of these dominating systematics.

The low-frequency systematics posed the largest problem due to the majority of the variables in this survey having real frequencies in this regime. They are clearly dominated by the sidereal cycle and its aliases; the large peaks that pick up around 0.167 day are due to the ansatz routine not picking up frequencies at that harmonic. We were careful when reporting frequencies as real when they were within $0.1 \mathrm{day}^{-1}$ of these frequencies. For example, if independent evidence of variability existed for these frequencies near a systematic (e.g., the eclipsing binary (EB) V397 Pup with a 3.00402 day period;
Watson et al. 2006), they were accepted as real. However, potential new variables could have been missed due to the lack of a sophisticated means of independent verification or imperfections in the detrending or ansatz routines. The highfrequency systematics were only applicable to the $\delta$ Scuti candidates due to their high-frequency regime; however, the systematics were not an issue for the $\delta$ Scuti primary frequencies detected in this study.

\subsection{Performance Analysis}

The sample from this study was also used to study the performance of bRing. In Figure 3, the rms for each postdetrending star was plotted in gray against the catalog magnitude of the star. For each camera, $\sim 14 \%$ of the stars performed better than $1 \%$ and $\sim 70 \%$ stars performed better than $2 \%$ (dashed line). The results here are similar to the results from Talens et al. (2017b, 2018).

By visual inspection, it is clear that a combined noise floor (plotted as a horizontal dotted-dashed line in Figure 3) exists in all 4 cameras at an rms of about 0.005 . The region brighter than $V \simeq 5.5 \mathrm{mag}$ is dominated by this combined noise floor term. Contributing terms to this noise floor include scintillation noise (estimated to be around $10^{-4}$ at both sites via Young's approximation: Young 1967; Osborn et al. 2015), noise contributed from the calibration and detrending, as well as other noise sources such as read noise and dark current. This noise floor level matches the expected photometric precision for bright stars in bRing, indicating that the detrending routine used in this work was successful (Stuik et al. 2017; Talens et al. $2017 \mathrm{~b}, 2018$ ). The fainter region was dominated by the shot noise and sky noise contributions. Overall, bRing performed as expected at the bright end and performed well for stars at the faint end, which made a complete survey of all the stars in the bRing data possible despite lingering systematics.

\section{Results and Discussion}

We detected 353 variable stars in the bRing survey. We used the VSX ${ }^{11}$ catalog, Vizier, ${ }^{12}$ and SIMBAD ${ }^{13}$ web services to identify previously known or candidate variables (Ochsenbein et al. 2000; Wenger et al. 2000; Watson et al. 2006). The periods reported for previously known variables were then compared to the periods detected with bRing. Stars that had no mention as variable stars in these databases, or suspected variables that did not have quoted periods in any source, are reported here as new periodic variables. Of the 284 previously known variables in this survey, the bRing periods were found to be consistent for $62 \%$ of the stars. The majority of the inconsistent periods were $\delta$ Scutis or long-period variables (LPVs). bRing could simply be detecting a more significant period or alias for the $\delta$ Scutis due to their multi-periodic nature that requires further study to disentangle (out of the scope of this work). The LPVs typically had low-precision measurements of the period, leading to the inconsistencies observed between previous measurements and bRing measurements.

bRing detected 71 variables that had not been previously flagged as known or candidate variables (including the 17 irregular variables observed by bRing). bRing was also able to reclassify four stars based on their newly measured period, light-curve shape,

\footnotetext{
11 The VSX catalog is regularly updated at https://www.aavso.org/vsx/.

12 https://vizier.u-strasbg.fr/

${ }^{13}$ http://simbad.u-strasbg.fr/simbad/
} 

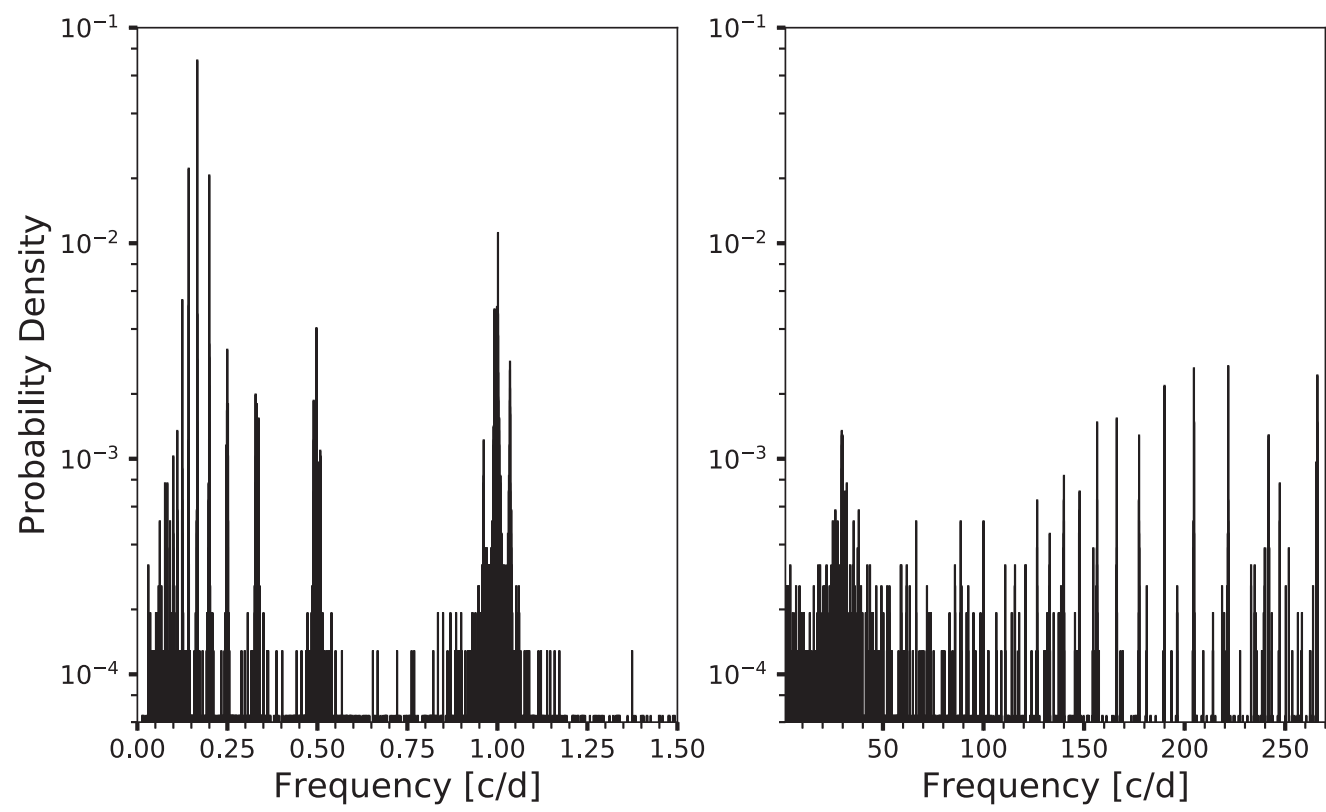

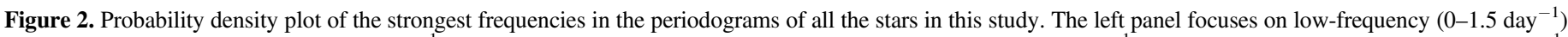

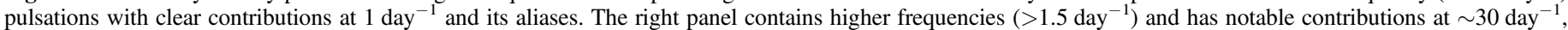

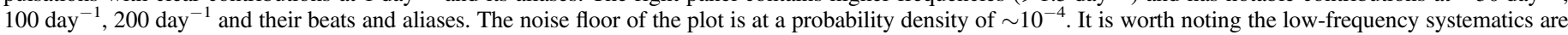
at least an order of magnitude stronger than the high-frequency systematics, which are themselves an order of magnitude stronger than the noise floor.

and spectral classification. The remainder of the stars showed no detectable or independent signs of variability down to the $\sim 1$ mmag level. These stars are tabulated by variable classification in the following subsections.

The color-absolute magnitude positions of the 353 variable stars are plotted in Figure 4. The different types of variables are symbol-coded with respect to the tables they inhabit in Section 4. Previously known variables are outlined in black, while newly identified variables are solid black. For the four stars that are reclassified in this work, a small black star was placed on top of their respective symbols. The SIMBAD service was queried for Johnson $B V$ photometry (Perryman \& ESA 1997; Kharchenko 2001), Galactic coordinates $l$ and $b$, and in the vast majority of cases, either a Gaia DR2 or Hipparcos parallaxes (Ochsenbein et al. 2000; van Leeuwen 2007; Brown et al. 2018). We queried the most recent $3 \mathrm{D}$ reddening maps from the STILISM $^{14}$ program to deredden the $(B-V)$ colors (Capitanio et al. 2017; Lallement et al. 2018). Following Mellon et al. (2019a), we adopted the ratio of total to selective extinction to be $A_{V} / E(B-V) \simeq 3.07+0.167(B-V)_{o}$, which is an adequate approximation over the intrinsic color interval $-0.32<$ $(B-V)_{o}<1.5$. Solar composition PARSEC isochrones (Bressan et al. 2012; Marigo et al. 2017) were overlaid for several ages; these were generated using the CMD 3.3 Input tool. ${ }^{15}$ The color-absolute magnitude parameters calculated for Figure 4 are tabulated in Table 10 in the Appendix.

\subsection{Cepheid Variables}

A total of 47 previously classified Cepheid variables detected with bRing have well-defined periods in the VSX catalog. These are tabulated in Table 1, which includes identification information about each star, the primary bRing period and amplitude, and the reported VSX period. This structure is used

\footnotetext{
14 https://stilism.obspm.fr/

15 http://stev.oapd.inaf.it/cgi-bin/cmd
}

for tables throughout this paper. The primary frequencies recovered by bRing agreed with all of the fundamental modes reported in the VSX catalog. A future study of Cepheids in bRing could yield fainter frequency modes present in their power spectrum and help identify the Blažhko effect if present (Blažko 1907). Based on their CMD position (Figure 4), two of the stars are unusual for Cepheids; we reclassify them.

HD 132247 (ASAS J145955-4957.9) is a A0IV star (Houk 1978) classified in VSX as both a first-overtone classical Cepheid and an $\alpha^{2}$ Canum Venaticorum (ACV) (Sitek \& Pojmański 2014). This is a poorly studied star that does show an 8 mmag pulsation at a period of 2.123 days. There are other modes present in the star's periodogram; however, nothing is indicative of it being a classical Cepheid in addition to its spectral classification. Although its period could indicate this is an ACV variable, a lack of spectral observations to identify chemical peculiarities and spectral line intensity variations make it challenging to unambiguously classify.

One possible variable classification is a $\delta$ Scuti. Although the luminosity class of the star suggests it lies beyond the blue edge of the instability strip (Breger \& Pamyatnykh 1998), its position in Figure $4\left((B-V)_{0}=0.13, M_{V}=1.40\right)$ is on top of the other $\delta$ Scutis in this study. In addition, $\delta$ Scutis have been shown to exist blueward of this theoretical limit (Bowman \& Kurtz 2018; Mellon et al. 2019a). Therefore, the classical Cepheid designation should be removed. The ACV designation should also be changed due to lack of a detailed spectral study. It is reasonable to suggest that this star is actually a $\delta$ Scuti based on its CMD position and multiple pulsation modes present in its periodogram.

HD 136633 (ASAS J152459-6156.7) is a B3V star (Houk \& Cowley 1975) classified as a fundamental-mode classical Cepheid in VSX (from Sitek \& Pojmański 2014). The periodogram does reveal multiple modes present; however, the B3V spectral classification means this star astrophysically is unlikely to be a classical Cepheid. It is more likely to be a $\beta$ 

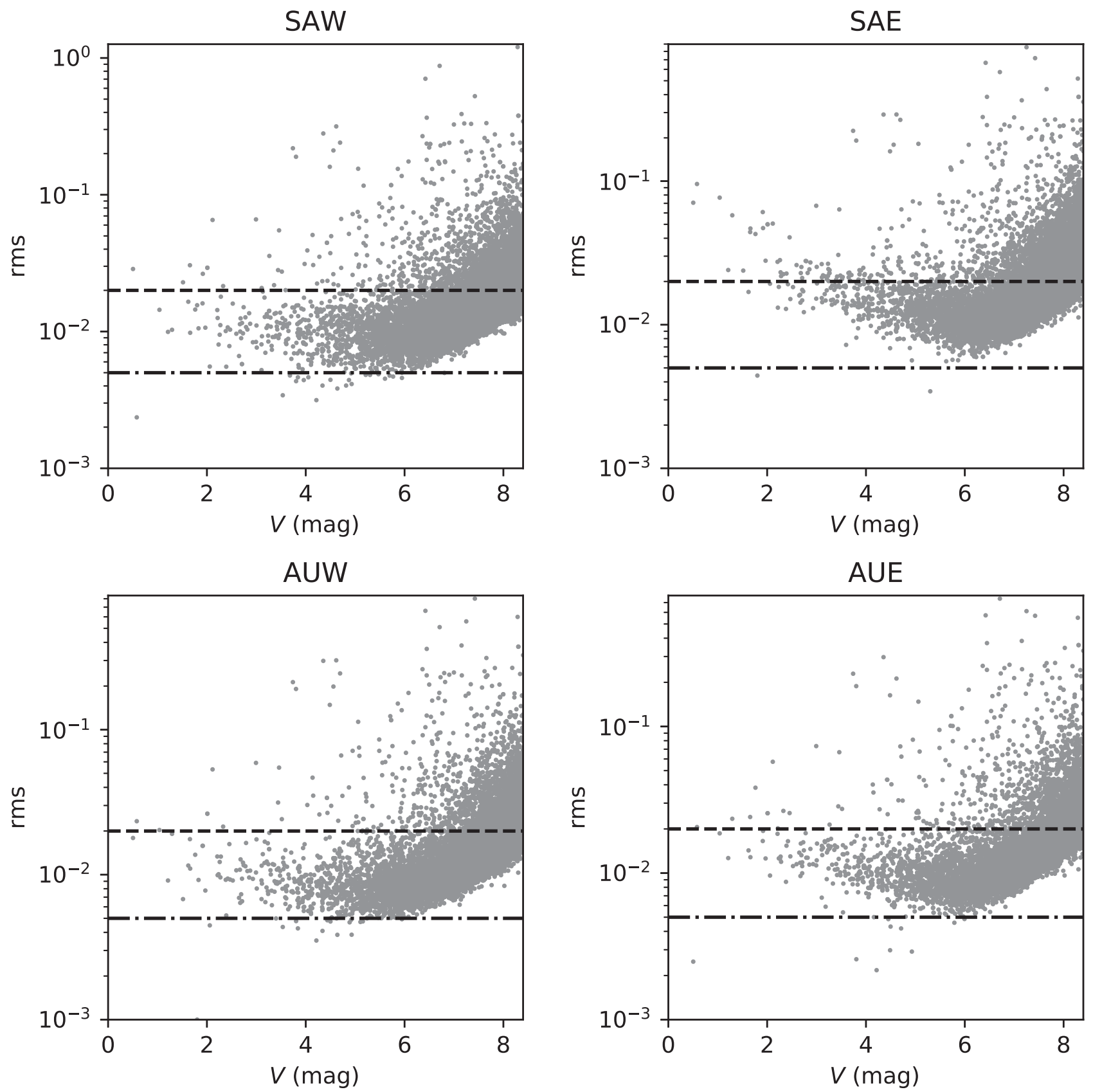

Figure 3. Plots showing the rms (gray dots) in each of the cameras for the 16,762 stars in this survey. The horizontal dashed line represents $2 \%$ scatter. The horizontal dotted-dashed line represents the combined noise floor estimated by visual inspection to be at an rms of around 0.005 .

Cephei (BCEP) or slowly pulsating B-type (SPB) star. This agrees with its position on the CMD (Figure 4: $(B-V)_{0}=$ $\left.-0.12, M_{V}=-2.00\right)$. The modes present in this star appear to better fit the description of an SPB and should be reclassified as such (De Cat 2007; Miglio et al. 2007).

\subsection{EBs and the O'Connell Effect}

We detected 120 EBs in the bRing data set. For most of these EBs, the periodogram revealed the half period (the phasefolded light curve showed the primary and secondary eclipses overlapping) as the dominating sinusoidal component. When a potential EB was found, the periodogram was rescanned in a window around double the original period to find the true period. For a few EBs, this was not true and the correct period was searched for manually. The known EBs were discussed in Section 4.2.1 and are tabulated in Table 2 like the Cepheids in Table 1. Periods from Rimoldini et al. (2012) were used in place of missing VSX periods where available. Three new EBs are discussed in Section 4.2.2 and summarized in Table 3. Eighteen of the bRing EB light curves also showed evidence of the O'Connell effect (O'Connell 1951), and these are discussed in Section 4.2.3 and their parameters are summarized in Table 4.

\subsubsection{Previously Identified EBs}

For the 117 variable stars previously classified as EBs, the measured periods were compared to the values listed in the VSX catalog. The periods we measured with bRing agreed with 


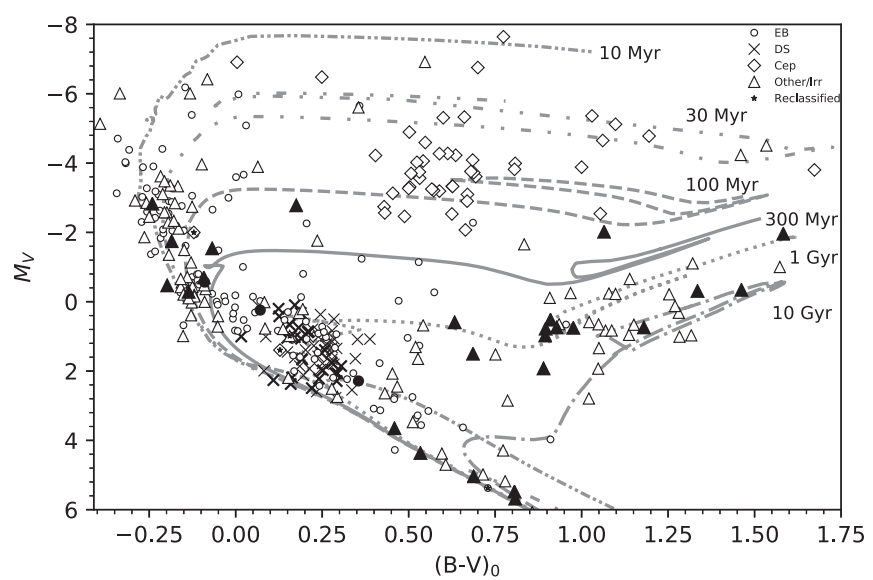

Figure 4. Color-magnitude diagram of the variables in this work. Types of variables are symbol-coded with respect to the tables presented in Section 4. The symbols for previously identified variables are outlined in black and newly identified variables are solid black. The four reclassified variables in this work are denoted with a black star. Several solar composition PARSEC isochrones are overlaid (Bressan et al. 2012; Marigo et al. 2017). Two of the stars had reddening values that placed them outside the scope of this plot; these stars were not plotted in order to better focus on the majority of the stars HD 85871 $\left((B-V)_{0}=2.32, M_{V}=-3.45\right)$ and $\operatorname{HD} 30551\left((B-V)_{0}=2.63, M_{V}=\right.$ -3.99). Another star (HD 69342) was not included because a distance could not be determined for this star.

$95(\sim 81 \%)$ of the periods reported in VSX within $1 \%$. There were 9 stars $(\sim 7 \%)$ whose bRing periods were double the VSX period, but for which we confirmed the bRing periods by visual inspection of the phase-folded light curve (DV Gru, BR Ind, V452 Car, HD 66623, HD 205877, HD 56910, V376 Pup, HR Lup, R Ara). For eight stars $(\sim 7 \%)$ a different period was detected that reproduces the eclipse structure, whereas the VSX period does not (V361 Pup, HD 70999, V360 Pup, V2509 Sgr, V661 Car, HD 16589, NO Pup, HD 203244). There were also five stars $(\sim 5 \%)$ whose bRing periods recovered the eclipsing structure at half the reported VSX period (DE Mic, HD 129094, X Car, V535 Ara, V831 Cen). We further discuss a couple of notable cases: HD 70999 and HD 203244.

$H D$ 70999: unfortunately, the VSX period for this star was near one of the strong, low-frequency bRing systematic false positives (see Section 3.3). The light curve also seemed to be missing the eclipses for $\sim 25 \%$ of the observations. When phasefolding on the VSX period of 2.99250 days, the phase-folded light curve showed the dip broken up into three segments with no clear eclipse structure. When bRing data were phase-folded to 1.9952 days, two dips were recovered, but no clear eclipse structure was seen. Due to this lack of data in bRing, the period for this eclipsing system was not accurately determined.

HD 203244 was classified as an Algol eclipsing binary (EA) in both the VSX catalog and Rimoldini et al. (2012), with the latter reporting an unusually long period of 833.29734 days. We detect in the bRing photometry a very strong period at 12.77751 days; however, the phase-folded light curve at this period is shallow and does not show a secondary eclipse as expected from an EA. Phasefolding on the half or double period did not reveal additional structure. HD 203244 is most likely an ellipsoidal variable based on the period and shape of the phase-folded light curve.

\subsubsection{New EBS}

Three new EBs were identified and their phase-folded light curves are shown in Figure 5. To better identify the orbital periods for these new EBs, a BLS routine adopted from other works in this group was used (e.g., Talens et al. 2017a; Dorval et al. 2019).

HD 77669: this is a B9III/IV star (Houk 1978) with $V$ magnitude 8.11 (Perryman \& ESA 1997) and parallax $\varpi=$ $1.9553 \pm 0.0514$ mas, corresponding to distance $d=511 \pm$ 13 pc (Gaia DR2 5331845690580305920; Brown et al. 2018). We detect a strong period of 7.70766 days with a primary eclipse depth of $0.18 \mathrm{mag}$ and a secondary eclipse depth of 0.15 mag. The observed transit depths in bRing are observed to be $>0.15 \mathrm{mag}$; however, the bottom of the primary and secondary eclipses are too deep for bRing to accurately measure.

HD 142049 (HR 5900): HD 142049 is cataloged in VSX as a suspected variable (NSV 7318). The Washington Double Star catalog (Mason et al. 2019) reports HD 142049 as a 4." 8 binary with $V$ magnitudes of 5.91 and 8.36, and the common motion and parallax of the pair is obvious in Gaia DR2 (Gaia DR2 5833110434699732352 and Gaia DR2 5833110434673386240 ; Brown et al. 2018). The Gaia DR2 parallaxes are $18.1136 \pm$ 0.0678 mas and $18.1238 \pm 0.0445$ mas for A and B, respectively, showing the resolved pair to be at distance $55.2 \mathrm{pc}$. The spectral types of the components are a matter of some contention, with Houk \& Cowley (1975) reporting components of type G5II/III and A3, noting that there is "slight possibility there is a Am or Fm star component," and Corbally (1984) reporting types of $\mathrm{kA} 3 \mathrm{hF} 3 \mathrm{mF} 4$ for the primary and F9.5V for the secondary. The bRing data show that the unresolved light from the system is consistent with a grazing EB with period 13.22062 day, with primary eclipse depth 0.050 mag and secondary eclipse depth of $0.045 \mathrm{mag}$. The binary must have a fairly eccentric orbit, as the eclipses are only 0.3 phase apart.

HD 155781: this $V=7.43$ star has spectral type A3IV/V (Houk \& Cowley 1975; Perryman \& ESA 1997) and parallax $3.9861 \pm 0.0561 \mathrm{mas}$, corresponding to distance $d=250.9 \pm$ 3.5 pc (Gaia DR2 5913908252773468928; Brown et al. 2018). We detect a strong signal at a period of 13.08670 days that appears to correspond to the orbital period of an EB with primary dips of $0.10 \mathrm{mag}$ and secondary dips of $0.08 \mathrm{mag}$.

\subsubsection{The O'Connell Effect}

This survey searched for evidence of the O'Connell effect in all of the W UMa and $\beta$ Lyr EBs in this data set. In W UMa and $\beta$ Lyr EBs, the O'Connell effect is observed as an asymmetry in the maximum brightness in between the primary and secondary eclipses, i.e., the maximum before the primary eclipse is fainter than the maximum before the secondary eclipse (O'Connell 1951). The underlying physical mechanism is not well understood (plausible explanations include surface features and Doppler beaming: Wilsey \& Beaky 2009; da Silva et al. 2014) though several examples have been detected (Pribulla et al. 2003, 2011; Burggraaff et al. 2018).

The O'Connell effect was detected in 18 of the bRing EBs, which have been tabulated in Table 4 . The differences between the maxima were considered significant if they exceeded $3 \sigma_{A}$, where $\sigma_{A}$ is the uncertainty in the amplitude of the EB. Only two of the EBs in this table (TY Men (Nagy 1985; Pribulla et al. 2011) and TU Mus (Terrell et al. 2003) have been noted in the literature as having evidence of asymmetry in their light curves. The other 16 have likely been missed due to the faint effect observed in bRing and possible variability of the effect 
Table 1

Previously Classified Cepheid Variables Detected with bRing

\begin{tabular}{|c|c|c|c|c|c|c|c|c|c|c|}
\hline $\begin{array}{l}\text { Name } \\
\ldots\end{array}$ & $\begin{array}{c}\mathrm{HD} \\
\cdots\end{array}$ & $\begin{array}{c}P \\
\text { (day) }\end{array}$ & $\begin{array}{c}\sigma_{P} \\
\text { (day) }\end{array}$ & $\begin{array}{c}A \\
\text { (mmag) }\end{array}$ & $\begin{array}{c}\sigma_{A} \\
\text { (mmag) }\end{array}$ & $\begin{array}{c}\text { VSX ID } \\
\ldots\end{array}$ & $\begin{array}{l}P_{\text {VSX }} \\
\text { (day) }\end{array}$ & $\begin{array}{c}V \\
(\mathrm{mag})\end{array}$ & $\begin{array}{r}\mathrm{SpT} \\
\ldots\end{array}$ & $\begin{array}{c}\text { References } \\
\ldots\end{array}$ \\
\hline bet Dor & 37350 & 9.844 & 0.007 & 157.8 & 1.6 & 13671 & 9.843 & 3.80 & F6Ia & 1 \\
\hline AP Pup & 65592 & 5.085 & 0.003 & 127.8 & 2.6 & 26671 & 5.084 & 7.34 & F8II & 2 \\
\hline AX Vel & 68556 & 2.592 & 0.001 & 82.8 & 1.5 & 37493 & 2.593 & 8.14 & F6II & 3 \\
\hline AH Vel & 68808 & 4.229 & 0.033 & 87.5 & 1.2 & 37478 & 4.227 & 5.73 & F7IB/II & 3 \\
\hline RS Pup & 68860 & 41.193 & 0.429 & 242.5 & 5.9 & 26613 & 41.443 & 7.00 & F8Iab & 2 \\
\hline V Car & 72275 & 6.699 & 0.003 & 143.0 & 1.7 & 5758 & 6.697 & 7.30 & $\mathrm{~F} 8 \mathrm{Ib} / \mathrm{II}$ & 1 \\
\hline RZ Vel & 73502 & 20.482 & 0.067 & 264.4 & 4.0 & 37434 & 20.398 & 7.15 & $\mathrm{G} 1 \mathrm{Ib}$ & 3 \\
\hline SW Vel & 74712 & 23.381 & 0.067 & 251.7 & 3.9 & 37439 & 23.407 & 8.30 & F8/G0Ib & 3 \\
\hline SX Vel & 74884 & 9.537 & 0.013 & 169.7 & 2.6 & 37440 & 9.550 & 8.34 & F8II & 3 \\
\hline BG Vel & 78801 & 6.928 & 0.01 & 97.0 & 1.4 & 37501 & 6.924 & 7.68 & F7/F8II & 3 \\
\hline V Vel & 81222 & 4.366 & 0.004 & 144.2 & 1.8 & 37421 & 4.371 & 7.57 & F8II & 1 \\
\hline I Car & 84810 & 35.688 & 0.060 & 167.4 & 1.9 & 6330 & 35.552 & 3.74 & G5Iab/Ib & 1 \\
\hline V397 Car & 87072 & 2.063 & 0.001 & 49.6 & 0.6 & 6150 & 2.063 & 8.30 & F8IB/II & 1 \\
\hline RY Vel & 89841 & 27.952 & 0.177 & 201.2 & 3.2 & 37433 & 28.136 & 8.40 & F5Ib/II & 1 \\
\hline VY Car & 93203 & 18.852 & 0.033 & 160.4 & 2.3 & 5796 & 18.890 & 7.62 & F7Iab/Ib & 1 \\
\hline U Car & 95109 & 38.609 & 0.315 & 247.6 & 3.3 & 5757 & 38.829 & 6.45 & G3Ia & 1 \\
\hline ER Car & 97082 & 7.722 & 0.011 & 103.0 & 1.4 & 5914 & 7.719 & 6.82 & G1Iab/Ib & 1 \\
\hline IT Car & 97485 & 7.524 & 0.008 & 68.1 & 1.0 & 5990 & 7.533 & 8.11 & F8Iab/b & 1 \\
\hline V419 Cen & 100148 & 5.502 & 0.005 & 61.7 & 1.0 & 7716 & 5.507 & 8.18 & F7II & 1 \\
\hline S Mus & 106111 & 9.689 & 0.012 & 108.3 & 1.4 & 19678 & 9.660 & 6.08 & F6Ib & 1 \\
\hline R Cru & 107805 & 5.818 & 0.004 & 140.4 & 2.1 & 10769 & 5.826 & 6.90 & $\mathrm{~F} 7 \mathrm{Ib} / \mathrm{II}$ & 1 \\
\hline BG Cru & 108968 & 3.345 & 0.002 & 45.4 & 0.6 & 10853 & 3.343 & 5.49 & F5III & 1 \\
\hline AG Cru & 110258 & 3.836 & 0.004 & 89.5 & 1.5 & 10829 & 3.837 & 8.23 & F8Ib/II & 1 \\
\hline R Mus & 110311 & 7.529 & 0.010 & 196.7 & 2.1 & 19677 & 7.510 & 7.51 & $\mathrm{~F} 7 \mathrm{Ib}$ & 1 \\
\hline S Cru & 112044 & 4.687 & 0.001 & 146.9 & 2.0 & 10770 & 4.690 & 6.73 & $\mathrm{~F} 7 \mathrm{Ib} / \mathrm{II}$ & 1 \\
\hline V659 Cen & 117399 & 5.629 & 0.007 & 59.4 & 0.8 & 7956 & 5.623 & 6.65 & F6/F7Ib & 1 \\
\hline XX Cen & 118769 & 10.938 & 0.021 & 171.5 & 2.4 & 7346 & 10.953 & 7.83 & F7/F8II & 1 \\
\hline V381 Cen & 120400 & 5.080 & 0.002 & 151.2 & 2.1 & 7678 & 5.079 & 7.68 & F8Ib/II & 1 \\
\hline V Cen & 127297 & 5.482 & 0.003 & 132.6 & 2.1 & 7302 & 5.494 & 6.80 & F5Ia & 1 \\
\hline AV Cir & 130233 & 3.066 & 0.002 & 74.6 & 0.8 & 9474 & 3.065 & 7.44 & F7II & 1 \\
\hline AX Cir & 130701 & 5.279 & 0.015 & 76.1 & 1.1 & 9476 & 5.273 & 5.94 & $\mathrm{~F} 8 \mathrm{II}+\mathrm{A} / \mathrm{F}$ & 1 \\
\hline$\cdots$ & $132247^{\mathrm{a}}$ & 2.123 & 0.008 & 8.7 & 0.2 & 412415 & 2.122 & 8.10 & A0IV & 3 \\
\hline $\mathrm{R} \operatorname{Tr} \mathrm{A}$ & 135592 & 3.392 & 0.002 & 129.8 & 1.3 & 36665 & 3.389 & 6.70 & $\mathrm{~F} 7 \mathrm{Ib} / \mathrm{II}$ & 1 \\
\hline$\ldots$ & $136633^{\mathrm{a}}$ & 6.118 & 0.009 & 28.0 & 0.5 & 412524 & 6.125 & 8.21 & B3V & 1 \\
\hline LR TrA & 137626 & 2.429 & 0.001 & 32.3 & 0.4 & 36930 & 2.428 & 7.79 & F8II & 1 \\
\hline$S$ TrA & 142941 & 6.324 & 0.006 & 162.3 & 1.8 & 36666 & 6.324 & 6.45 & F8II & 1 \\
\hline $\mathrm{U}$ TrA & 143999 & 2.567 & 0.002 & 151.4 & 1.8 & 36668 & 2.568 & 7.92 & $\mathrm{~F} 8 \mathrm{Ib} / \mathrm{II}$ & 1 \\
\hline S Nor & 146323 & 9.754 & 0.018 & 84.1 & 1.0 & 19962 & 9.754 & 6.53 & F8/G0Ib & 1 \\
\hline RV Sco & 153004 & 6.067 & 0.011 & 155.3 & 5.0 & 32830 & 6.061 & 7.16 & G0Ib & 2 \\
\hline V636 Sco & 156979 & 6.803 & 0.010 & 93.5 & 1.5 & 33452 & 6.797 & 6.68 & $\mathrm{~F} 7 / \mathrm{F} 8 \mathrm{Ib} / \mathrm{II}$ & 3 \\
\hline V482 Sco & 158443 & 4.529 & 0.007 & 106.0 & 3.5 & 33298 & 4.528 & 7.93 & F8/G0II & 2 \\
\hline V950 Sco & 159654 & 3.378 & 0.001 & 67.0 & 1.1 & 33766 & 3.380 & 7.27 & $\mathrm{~F} 5 \mathrm{Ib}$ & 3 \\
\hline X Sgr & 161592 & 7.018 & 0.003 & 278.0 & 8.6 & 27707 & 7.013 & 4.56 & F7II & 2 \\
\hline RY Sco & 162102 & 20.063 & 0.007 & 172.8 & 5.1 & 32833 & 20.323 & 8.18 & F6Ib & 2 \\
\hline W Sgr & 164975 & 7.597 & 0.001 & 172.4 & 6.9 & 27706 & 7.595 & 4.70 & G0Ib/II & 2 \\
\hline kap Pav & 174694 & 9.031 & 0.008 & 180.9 & 2.1 & 25119 & 9.083 & 4.36 & F5Ib-II: & 1 \\
\hline XY Car & 308149 & 12.430 & 0.055 & 5.5 & 0.1 & 5803 & 12.434 & 6.97 & A9Ib-II & 1 \\
\hline
\end{tabular}

Note.

${ }^{a}$ Reclassified in this work, see Section 4.1.

References. (1) Houk \& Cowley (1975), (2) Houk (1982), (3) Houk (1978).

(Wilsey \& Beaky 2009) masking the asymmetry in previous studies.

\section{3. $\delta$ Scuti Variables}

We detected $66 \delta$ Scuti variables in the bRing data set, 26 of which are candidates that had not been previously reported as detected pulsators. For the 40 previously known $\delta$ Scutis, we report only the strongest frequency in the bRing light curve. The previously published frequencies for the $\delta$ Scuti variables in Table 5 are from VSX by default; however, if one was not listed in VSX, we cite additional sources (Rodríguez et al. 2000; Rimoldini et al. 2012; Mellon et al. 2019a). The reported periods for $22(55 \%)$ of the $\delta$ Scuti variables in Table 5 do not match those reported in previous studies. All of the $\delta$ Scutis are very tightly bound with their positions on the CMD (Figure 4; this is useful for confirming the new $\delta$ Scutis candidates by inspection. The bright $\delta$ Scuti variable $\beta$ Pictoris itself was not included in this work because the bRing data for $\beta$ Pictoris 
Table 2

Previously Classified Eclipsing Binaries Detected with bRing

\begin{tabular}{|c|c|c|c|c|c|c|c|c|c|c|}
\hline $\begin{array}{l}\text { Name } \\
\ldots\end{array}$ & $\begin{array}{c}\mathrm{HD} \\
\ldots\end{array}$ & $\begin{array}{c}P \\
\text { (day) }\end{array}$ & $\begin{array}{c}\sigma_{P} \\
\text { (day) }\end{array}$ & $\begin{array}{c}A \\
(\mathrm{mmag})\end{array}$ & $\begin{array}{c}\sigma_{A} \\
(\mathrm{mmag})\end{array}$ & $\begin{array}{c}\text { VSX ID } \\
\ldots\end{array}$ & $\begin{array}{l}P_{\text {VSX }} \\
\text { (day) }\end{array}$ & $\begin{array}{c}V \\
(\mathrm{mag})\end{array}$ & $\begin{array}{ll}\text { SpT } & \\
& \ldots\end{array}$ & $\begin{array}{c}\text { References } \\
\ldots\end{array}$ \\
\hline zet Phe & 6882 & 1.66985 & $1 \mathrm{e}-04$ & 36.7 & 0.5 & 26329 & 1.66978 & 3.98 & $\mathrm{~B} 6 \mathrm{~V}+\mathrm{B} 0 \mathrm{~V}$ & 1 \\
\hline$\ldots$ & 16589 & 6.33296 & $2 \mathrm{e}-04$ & 5.4 & 0.2 & 53991 & $0.82414^{\mathrm{a}}$ & 6.48 & F6V & 2 \\
\hline CN Hyi & 17653 & 0.45609 & $2 \mathrm{e}-04$ & 68.6 & 0.6 & 16473 & 0.45611 & 6.67 & F6V & 1 \\
\hline WZ Hor & 17755 & 0.72886 & $1 \mathrm{e}-04$ & 48.0 & 0.6 & 15947 & 0.72885 & 8.06 & F3/F5V & 1 \\
\hline VY Ret & 21765 & 14.21605 & $5 e-04$ & 3.3 & 0.2 & 39786 & 14.21605 & 7.89 & F5V & 1 \\
\hline $\mathrm{RZ} \mathrm{Cae}$ & 29087 & 2.48712 & $5 e-04$ & 10.2 & 0.4 & 4529 & 2.48696 & 7.83 & A4V & 2 \\
\hline AN Dor & 31407 & 2.03274 & $1 \mathrm{e}-04$ & 13.2 & 0.3 & 13656 & 2.03268 & 7.67 & $\mathrm{~B} 2 / \mathrm{B} 3 \mathrm{~V}$ & 1 \\
\hline AR Dor & 34349 & 2.95130 & $8 \mathrm{e}-05$ & 4.7 & 0.1 & 13660 & 2.95206 & 7.03 & F5V & 1 \\
\hline UX Men & 37513 & 4.18110 & $1 e-03$ & 25.5 & 0.8 & 18670 & 4.18110 & 8.25 & F8V & 1 \\
\hline TY Men & 37909 & 0.46166 & $1 e-04$ & 99.0 & 1.0 & 18665 & 0.46167 & 8.26 & A3/A4V & 1 \\
\hline del Pic & 42933 & 1.67248 & $2 \mathrm{e}-04$ & 46.9 & 0.6 & 26396 & 1.67254 & 4.72 & B0.5IV & 1 \\
\hline V360 Pup & 52993 & 1.12803 & $2 \mathrm{e}-04$ & 10.7 & 0.3 & 26962 & 1.29644 & 6.57 & $\mathrm{ApSi}$ & 2 \\
\hline V361 Pup & 54579 & 0.23661 & $5 e-04$ & 49.8 & 2.7 & 26963 & 0.36737 & 8.04 & G0V & 3 \\
\hline $\mathrm{FF} \mathrm{CMa}$ & 55173 & 1.21332 & $4 \mathrm{e}-04$ & 67.2 & 3.2 & 5323 & 1.21337 & 7.48 & $\mathrm{~B} 3 / 5 \mathrm{~V}(\mathrm{p})$ & 2 \\
\hline V452 Car & 56146 & 2.11033 & $1 \mathrm{e}-05$ & 23.2 & 0.4 & 6205 & 1.05502 & 8.10 & B8IV & 1 \\
\hline$\cdots$ & 56910 & 1.83724 & $2 \mathrm{e}-05$ & 5.7 & 0.2 & 55845 & $0.94929^{\mathrm{a}}$ & 6.84 & $\mathrm{~A} 2 / 3 \mathrm{~mA} 4-\mathrm{A} 7$ & 1 \\
\hline V376 Pup & 60559 & 3.88333 & $2 \mathrm{e}-04$ & 3.8 & 0.2 & 26978 & 1.94270 & 6.25 & B8IV(p Si) & 2 \\
\hline V454 Car & 60649 & 0.98049 & $1 \mathrm{e}-04$ & 32.6 & 0.5 & 6207 & 0.98042 & 6.99 & B4/B5V & 1 \\
\hline V455 Car & 61644 & 5.13038 & $2 \mathrm{e}-04$ & 14.4 & 0.3 & 6208 & 5.13300 & 8.40 & B5/B6IV & 1 \\
\hline V606 Car & 63203 & 12.31530 & $2 \mathrm{e}-03$ & 9.5 & 0.4 & 42349 & 12.31920 & 8.31 & B8/B9III & 1 \\
\hline V397 Pup & 63786 & 3.00402 & $2 \mathrm{e}-04$ & 3.4 & 0.2 & 26999 & 3.00445 & 5.93 & B9V & 2 \\
\hline QZ Pup & 64503 & 1.11207 & $2 \mathrm{e}-04$ & 8.4 & 0.2 & 26936 & 1.11203 & 4.48 & $\mathrm{~B} 2 \mathrm{~V}$ & 2 \\
\hline V Pup & 65818 & 1.45441 & $2 \mathrm{e}-04$ & 115.9 & 1.5 & 26607 & 1.45449 & 4.49 & $\mathrm{~B} 1 \mathrm{Vp}+\mathrm{B} 2$ & 4 \\
\hline$\cdots$ & 66623 & 0.85182 & $1 e-04$ & 7.6 & 0.6 & 250227 & 0.42573 & 8.11 & F7V & 2 \\
\hline V462 Car & 66768 & 1.10561 & $6 e-05$ & 30.7 & 0.4 & 6215 & 1.10569 & 6.71 & B3V(n) & 1 \\
\hline V431 Pup & 69882 & 9.34999 & $1 e-04$ & 10.9 & 0.3 & 27033 & 9.35928 & 7.18 & B1III: & 5 \\
\hline$\cdots$ & 70999 & 1.99520 & $5 e-03$ & 14.1 & 0.6 & 358580 & 2.99250 & 8.04 & B3III & 2 \\
\hline HR 3322 & 71302 & 4.93500 & $2 \mathrm{e}-04$ & 4.4 & 0.2 & 27040 & 4.93500 & 5.97 & $\mathrm{~B} 3 \mathrm{~V}$ & 5 \\
\hline NO Pup & 71487 & 0.77183 & $2 \mathrm{e}-03$ & 10.3 & 0.3 & 26892 & 1.25689 & 6.50 & B9IV/V & 2 \\
\hline XY Рух & 71801 & 0.92254 & $4 e-04$ & 10.1 & 0.4 & 27231 & 0.92254 & 5.74 & $\mathrm{~B} 2 \mathrm{~V}$ & 2 \\
\hline X Car & 72698 & 0.54132 & 1e-04 & 46.5 & 0.8 & 5760 & 1.08263 & 8.06 & A0Vn & 1 \\
\hline FY Vel & 72754 & 33.88620 & $5 e-04$ & 39.2 & 0.6 & 37604 & 33.72000 & 6.89 & B2Iape & 5 \\
\hline V470 Car & 72878 & 2.16177 & $2 \mathrm{e}-04$ & 19.9 & 0.4 & 6223 & 2.16178 & 7.47 & B9IV & 1 \\
\hline V454 Vel & 73699 & 1.13484 & $2 \mathrm{e}-04$ & 16.1 & 0.4 & 272444 & 1.13492 & 7.58 & $\mathrm{~B} 3 \mathrm{~V}$ & 2 \\
\hline NX Vel & 73882 & 2.91834 & $3 e-04$ & 6.0 & 0.3 & 37715 & 2.91988 & 7.26 & O8V: & 6 \\
\hline RS Cha & 75747 & 1.66999 & $1 \mathrm{e}-04$ & 51.3 & 0.6 & 9248 & 1.66987 & 6.08 & A7V & 1 \\
\hline CV Vel & 77464 & 6.89145 & $3 e-04$ & 2.1 & 0.1 & 37538 & 6.88949 & 6.70 & $\mathrm{~B} 2 \mathrm{~V}+\mathrm{B} 2 \mathrm{~V}$ & 5 \\
\hline GP Vel & 77581 & 8.97155 & $2 \mathrm{e}-04$ & 10.8 & 0.4 & 37614 & 8.964357 & 6.91 & $\mathrm{~B} 0.5 \mathrm{Ib}$ & 5 \\
\hline PQ Vel & 78165 & 22.2632 & $1 \mathrm{e}-03$ & 4.5 & 0.2 & 37731 & 22.2632 & 7.61 & $\mathrm{~A} 2 / 3 \mathrm{III}(\mathrm{m})$ & 5 \\
\hline V476 Car & 78763 & 1.28135 & $2 \mathrm{e}-03$ & 15.2 & 0.3 & 6229 & 1.28143 & 8.30 & B7Vn & 1 \\
\hline S Vel & 82829 & 5.93101 & $2 \mathrm{e}-03$ & 23.1 & 0.4 & 37418 & 5.93365 & 7.80 & $\mathrm{~A} 5 \mathrm{Ve}+\mathrm{K} 5 \mathrm{IIIe}$ & 7 \\
\hline IP Vel & 84400 & 3.43679 & $1 \mathrm{e}-04$ & 23.3 & 0.4 & 37649 & 3.43789 & 6.16 & B6V & 5 \\
\hline V486 Car & 84416 & 1.09378 & $1 \mathrm{e}-04$ & 32.7 & 0.4 & 6239 & 1.09389 & 6.32 & $\mathrm{~A} 0 \mathrm{~V}$ & 1 \\
\hline KN Vel & 85037 & 2.72327 & $1 \mathrm{e}-04$ & 7.9 & 0.2 & 37663 & 2.72290 & 6.52 & $\mathrm{~A} 2 \mathrm{IV}(\mathrm{m})$ & 5 \\
\hline QX Vel & 85185 & 0.87811 & $2 \mathrm{e}-04$ & 37.8 & 0.6 & 37748 & 0.87807 & 8.00 & $\mathrm{~A} 0 \mathrm{~V}$ & 5 \\
\hline QX Car & 86118 & 4.47804 & $1 \mathrm{e}-04$ & 14.0 & 0.3 & 6085 & 4.47804 & 6.66 & $\mathrm{~B} 3 \mathrm{~V}+\mathrm{B} 3 \mathrm{~V}$ & 1 \\
\hline V367 Car & 86441 & 5.71172 & $2 \mathrm{e}-04$ & 13.6 & 0.3 & 6120 & 5.73000 & 7.52 & B6V & 1 \\
\hline V341 Vel & 89611 & 14.73000 & $9 e-04$ & 2.8 & 0.3 & 37757 & 14.73000 & 7.96 & A0IV & 5 \\
\hline V435 Vel & 90000 & 10.49500 & $7 e-04$ & 4.7 & 0.2 & 37761 & 10.49500 & 7.56 & $\mathrm{~B} 3 \mathrm{~V}$ & 5 \\
\hline$\ldots$ & 90941 & 7.56760 & $4 \mathrm{e}-04$ & 1.1 & 0.2 & 411431 & 7.56470 & 7.87 & B4IV & 5 \\
\hline CC Ant & 91519 & 2.44594 & $6 e-05$ & 19.7 & 0.6 & 172655 & 2.44514 & 7.70 & A8III & 2 \\
\hline V661 Car & 93130 & 0.39875 & $1 \mathrm{e}-04$ & 14.8 & 0.4 & 56932 & 23.9438 & 8.08 & O6III & 6 \\
\hline RZ Cha & 93486 & 2.83200 & $2 \mathrm{e}-04$ & 24.8 & 0.4 & 9255 & 2.83208 & 8.08 & $\mathrm{~F} 5 \mathrm{~V}+\mathrm{F} 5$ & 1 \\
\hline V356 Vel & 93668 & 1.76804 & $2 \mathrm{e}-04$ & 14.6 & 0.3 & 37772 & 1.76791 & 6.74 & $\mathrm{~A} 0 \mathrm{~V}$ & 5 \\
\hline V772 Car & 94924 & 0.88419 & $2 \mathrm{e}-04$ & 27.4 & 0.4 & 172663 & 0.88417 & 8.01 & A1V & 1 \\
\hline V529 Car & 95993 & 4.74574 & $2 \mathrm{e}-04$ & 33.8 & 0.6 & 6282 & 4.74461 & 8.18 & $\mathrm{~B} 8 \mathrm{~V}$ & 1 \\
\hline TU Mus & 100213 & 1.38710 & $1 \mathrm{e}-04$ & 101.9 & 1.3 & 19704 & 1.38728 & 8.40 & $\mathrm{O} 8(+\mathrm{O} 8)$ & 6 \\
\hline V1101 Cen & 102682 & 5.03350 & $2 \mathrm{e}-04$ & 25.0 & 0.6 & 43976 & 5.03230 & 8.23 & F5V & 5 \\
\hline LZ Cen & 102893 & 2.75772 & $1 \mathrm{e}-04$ & 88.6 & 1.2 & 7571 & 2.75772 & 8.24 & B2III & 1 \\
\hline V788 Cen & 105509 & 4.96697 & $1 e-03$ & 1.9 & 0.1 & 8085 & 4.96638 & 5.74 & A3III & 5 \\
\hline V831 Cen & 114529 & 0.32142 & $5 e-03$ & 5.0 & 0.1 & 8128 & 0.64252 & 4.58 & $\mathrm{~B} 8 \mathrm{~V}$ & 1 \\
\hline V964 Cen & 115823 & 1.54308 & $1 \mathrm{e}-04$ & 6.5 & 0.1 & 8261 & 1.54259 & 5.45 & B6V & 5 \\
\hline V979 Cen & 119888 & 2.56882 & $7 e-05$ & 18.0 & 0.3 & 8276 & 2.56841 & 7.84 & B8II & 1 \\
\hline
\end{tabular}


Table 2

(Continued)

\begin{tabular}{|c|c|c|c|c|c|c|c|c|c|c|}
\hline $\begin{array}{l}\text { Name } \\
\ldots\end{array}$ & $\begin{array}{c}\mathrm{HD} \\
\ldots\end{array}$ & $\begin{array}{c}P \\
\text { (day) }\end{array}$ & $\begin{array}{c}\sigma_{P} \\
(\text { day })\end{array}$ & $\begin{array}{c}A \\
(\mathrm{mmag})\end{array}$ & $\begin{array}{c}\sigma_{A} \\
(\mathrm{mmag})\end{array}$ & $\begin{array}{c}\text { VSX ID } \\
\ldots\end{array}$ & $\begin{array}{l}P_{\text {VSX }} \\
\text { (day) }\end{array}$ & $\begin{array}{c}V \\
(\mathrm{mag})\end{array}$ & $\begin{array}{ll}\text { SpT } & \\
& \ldots\end{array}$ & $\begin{array}{c}\text { References } \\
\ldots\end{array}$ \\
\hline V1294 Cen & 121291 & 1.16556 & $2 \mathrm{e}-04$ & 25.4 & 0.5 & 45116 & 1.16553 & 7.89 & $\mathrm{~A} 0 \mathrm{Vn}+\mathrm{K} 2(\mathrm{III})$ & 5 \\
\hline AT Cir & 122314 & 3.25748 & $3 e-04$ & 9.8 & 0.2 & 9472 & 3.25749 & 7.62 & A5IV/Vs & 1 \\
\hline V992 Cen & 122844 & 1.21168 & $1 e-04$ & 16.4 & 0.3 & 8289 & 1.21156 & 6.20 & A5III/IV & 1 \\
\hline$\ldots$ & 123720 & 0.86872 & $5 e-05$ & 16.4 & 0.3 & 58490 & 0.86880 & 7.75 & $\mathrm{~A} 4 \mathrm{~V}$ & 1 \\
\hline V716 Cen & 124195 & 1.49024 & $2 \mathrm{e}-04$ & 32.9 & 0.5 & 8013 & 1.49010 & 6.09 & $\mathrm{~B} 5 \mathrm{~V}$ & 8 \\
\hline RR Cen & 124689 & 0.60570 & $1 e-04$ & 71.8 & 1.1 & 7307 & 0.60569 & 7.46 & A9/F0V & 1 \\
\hline$\ldots$ & 129094 & 0.39881 & $2 \mathrm{e}-03$ & 27.1 & 0.8 & 98784 & 0.74422 & 8.37 & F7V & 1 \\
\hline QZ Lup & 131638 & 1.13658 & $1 \mathrm{e}-04$ & 15.6 & 0.3 & 45479 & 1.13655 & 8.32 & B9V & 5 \\
\hline HR Lup & 133880 & 1.75470 & $1 e-04$ & 11.9 & 0.3 & 17811 & 0.87748 & 5.76 & B8IVSi & 9 \\
\hline del Cir & 135240 & 3.90445 & $1 \mathrm{e}-04$ & 19.2 & 0.3 & 9529 & 3.90248 & 5.07 & $08.5 \mathrm{~V}$ & 6 \\
\hline GG Lup & 135876 & 1.84961 & $1 e-03$ & 4.9 & 0.3 & 17783 & 1.84961 & 5.59 & B9V & 5 \\
\hline MP TrA & 143028 & 2.07017 & $3 e-04$ & 8.3 & 0.2 & 36942 & 2.06972 & 7.80 & $\mathrm{~B} 7 \mathrm{Ib} / \mathrm{II}$ & 1 \\
\hline V399 Nor & 147170 & 3.19301 & $3 e-04$ & 13.3 & 0.3 & 59034 & 3.19288 & 8.21 & F6/F7V & 3 \\
\hline V760 Sco & 147683 & 1.73074 & $2 \mathrm{e}-04$ & 15.1 & 0.6 & 33576 & 1.73090 & 7.05 & $\mathrm{~B} 4 \mathrm{~V}$ & 2 \\
\hline OT Aps & 148891 & 2.42603 & $9 \mathrm{e}-05$ & 6.7 & 0.2 & 832 & 2.42660 & 8.00 & B9.5IV & 1 \\
\hline V1288 Sco & 149450 & 1.10896 & $1 \mathrm{e}-05$ & 32.0 & 0.6 & 46471 & 1.10890 & 8.23 & B3III & 5 \\
\hline V882 Ara & 149668 & 20.96590 & $9 \mathrm{e}-05$ & 4.3 & 0.2 & 59156 & 20.96590 & 7.61 & A2IV & 1 \\
\hline $\mathrm{R}$ Ara & 149715 & 8.85166 & $2 \mathrm{e}-03$ & 25.7 & 0.8 & 2804 & 4.42522 & 8.33 & KOIII & 1 \\
\hline V954 Sco & 149779 & 1.26883 & $1 \mathrm{e}-04$ & 48.9 & 0.8 & 33770 & 1.26859 & 7.57 & B2IV & 5 \\
\hline V878 Ara & 151475 & 0.77053 & $6 e-05$ & 46.0 & 0.7 & 136724 & 0.77046 & 8.05 & B3II/III & 5 \\
\hline V1290 Sco & 151564 & 4.49267 & $2 \mathrm{e}-04$ & 6.0 & 0.3 & 59217 & 4.49244 & 7.98 & O9.5IV & 5 \\
\hline HR 6247 & 151890 & 1.44647 & $1 e-04$ & 44.8 & 0.8 & 34007 & 1.44627 & 2.99 & $\mathrm{~B} 1.5 \mathrm{IV}+\mathrm{B}$ & 10 \\
\hline V1295 Sco & 152333 & 2.15767 & $3 e-04$ & 32.4 & 0.6 & 59262 & 2.15767 & 8.07 & B1-2Ib-II & 5 \\
\hline V861 Sco & 152667 & 7.85382 & $1 \mathrm{e}-04$ & 38.4 & 0.8 & 33677 & 7.84818 & 6.18 & B0.5Ia & 2 \\
\hline V883 Sco & 152901 & 4.34113 & $1 e-04$ & 23.6 & 0.5 & 33699 & 4.34119 & 7.39 & $\mathrm{~B} 2.5 \mathrm{Vn}$ & 11 \\
\hline V836 Ara & 153140 & 7.04075 & $2 \mathrm{e}-04$ & 24.8 & 0.4 & 3639 & 7.03418 & 7.51 & B1II & 5 \\
\hline V616 Ara & 154339 & 4.99671 & $6 e-05$ & 52.4 & 0.8 & 3419 & 4.99525 & 8.26 & B3II/III & 5 \\
\hline FV Sco & 155550 & 5.72861 & $2 \mathrm{e}-04$ & 37.9 & 1.4 & 33001 & 5.72790 & 8.07 & B4IV & 2 \\
\hline V1012 Sco & 155775 & 1.51531 & $2 \mathrm{e}-04$ & 12.0 & 0.2 & 33828 & 1.51548 & 6.72 & B1V & 6 \\
\hline V499 Sco & 158155 & 2.33216 & $2 \mathrm{e}-04$ & 67.2 & 2.5 & 33315 & 2.33330 & 8.29 & B1III & 2 \\
\hline V1081 Sco & 158186 & 2.51419 & $1 \mathrm{e}-04$ & 10.5 & 0.6 & 33897 & 2.51374 & 7.00 & $09.5 \mathrm{~V}(\mathrm{n})$ & 6 \\
\hline V535 Ara & 159441 & 0.31466 & $2 \mathrm{e}-04$ & 30.2 & 0.4 & 3338 & 0.62930 & 7.36 & A8V & 1 \\
\hline V539 Ara & 161783 & 3.16836 & $3 e-04$ & 19.6 & 0.3 & 3342 & 3.16909 & 5.70 & $\mathrm{~B} 2 \mathrm{~V}+\mathrm{B} 3 \mathrm{~V}$ & 1 \\
\hline V453 Sco & 163181 & 12.00201 & $7 e-05$ & 82.7 & 2.4 & 33269 & 12.00597 & 6.60 & $09.5 \mathrm{Ia} / \mathrm{ab}$ & 2 \\
\hline V1647 Sgr & 163708 & 3.28277 & $1 \mathrm{e}-04$ & 24.5 & 1.0 & 29347 & 3.28279 & 7.06 & A3III & 2 \\
\hline V2509 Sgr & 167231 & 1.84197 & $4 \mathrm{e}-04$ & 34.9 & 1.4 & 30209 & 1.08697 & 7.41 & A0IV & 2 \\
\hline V681 CrA & 171577 & 4.32961 & $2 \mathrm{e}-04$ & 2.8 & 0.2 & 10552 & 4.32788 & 7.74 & B9V & 5 \\
\hline V362 Pav & 173344 & 2.74826 & $7 e-05$ & 5.3 & 0.1 & 25082 & 2.74844 & 7.39 & $\mathrm{~A} 2 \mathrm{mA5}-\mathrm{A} 9$ & 1 \\
\hline V363 Pav & 174139 & 1.19491 & $1 \mathrm{e}-04$ & 27.7 & 0.4 & 25083 & 1.19497 & 8.17 & $\mathrm{~B} 9 / \mathrm{B} 9.5 \mathrm{~V}$ & 1 \\
\hline V4407 Sgr & 174632 & 1.45165 & $1 \mathrm{e}-04$ & 19.1 & 0.8 & 32107 & 1.45174 & 6.64 & B7/B8IV & 8 \\
\hline$\ldots$ & 177776 & 1.65022 & $7 e-05$ & 12.7 & 0.3 & 414518 & 1.65006 & 8.12 & B9.5Vn & 1 \\
\hline V4089 Sgr & 184035 & 4.62891 & $3 e-04$ & 9.5 & 0.3 & 31789 & 4.62988 & 5.90 & A5IV-III & 5 \\
\hline HO Tel & 187418 & 1.61294 & $2 \mathrm{e}-04$ & 53.9 & 0.8 & 36458 & 1.61310 & 8.30 & $\mathrm{~A} 7 \mathrm{III}(\mathrm{m})$ & 5 \\
\hline V4437 Sgr & 193174 & 1.13654 & $3 e-04$ & 36.0 & 1.3 & 32137 & 1.13662 & 7.24 & A9IV/V & 2 \\
\hline V386 Pav & 198736 & 0.55187 & $2 \mathrm{e}-04$ & 31.2 & 0.4 & 25106 & 0.55184 & 8.34 & A9V & 1 \\
\hline DE Mic & 200670 & 0.20535 & $2 \mathrm{e}-04$ & 16.6 & 0.5 & 137558 & 0.41069 & 7.80 & $\mathrm{~F} 6 / 7 \mathrm{~V}$ & 2 \\
\hline BR Ind & 201427 & 1.78553 & $2 \mathrm{e}-04$ & 6.7 & 0.2 & 16577 & 0.89277 & 7.09 & F8V & 3 \\
\hline$\ldots$ & $203244^{\mathrm{b}}$ & 12.77751 & $7 e-03$ & 9.0 & 0.1 & 64006 & $833.29734^{\mathrm{a}}$ & 6.98 & G5V & 3 \\
\hline $\mathrm{CH}$ Ind & 204370 & 5.94788 & $2 \mathrm{e}-03$ & 12.9 & 0.3 & 137591 & 5.95320 & 7.52 & A9V & 5 \\
\hline$\ldots$ & 205877 & 7.68402 & $3 e-04$ & 7.4 & 0.1 & 64150 & $3.83266^{\mathrm{a}}$ & 6.20 & F7III & 5 \\
\hline CP Gru & 208614 & 2.08577 & $3 e-04$ & 29.1 & 0.4 & 14785 & 2.08615 & 7.72 & $\mathrm{~A} 5 \mathrm{~V}$ & 5 \\
\hline DV Gru & 210572 & 9.61553 & $1 \mathrm{e}-03$ & 2.9 & 0.1 & 64287 & 4.81803 & 7.72 & F8V & 1 \\
\hline DK Tuc & 212661 & 5.33386 & $4 \mathrm{e}-04$ & 5.2 & 0.2 & 37084 & 5.33793 & 6.90 & $\mathrm{~A} 1 \mathrm{mA5}-\mathrm{F} 0$ & 1 \\
\hline DP Gru & 220633 & 3.80231 & $3 e-04$ & 9.5 & 0.3 & 14807 & 3.80350 & 8.29 & F5/F6V & 5 \\
\hline
\end{tabular}

Notes.

${ }^{a}$ Rimoldini et al. (2012).

b Reclassified.

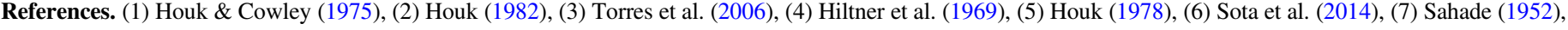
(8) Hube (1970), (9) Buscombe (1969), (10) Levato (1975), (11) Garrison et al. (1977). 
Table 3

New Eclipsing Binaries Detected with bRing

\begin{tabular}{lcccccccc}
\hline \hline $\begin{array}{l}\text { HD } \\
\cdots\end{array}$ & $\begin{array}{c}P \\
(\text { day })\end{array}$ & $\begin{array}{c}\sigma_{P} \\
(\text { day })\end{array}$ & $\begin{array}{c}\text { Pri } \\
(\mathrm{mag})\end{array}$ & $\begin{array}{c}\text { Sec } \\
(\mathrm{mag})\end{array}$ & $\begin{array}{c}\text { VSX ID } \\
\cdots\end{array}$ & $\begin{array}{c}V \\
(\mathrm{mag})\end{array}$ & $\begin{array}{c}\text { SpT } \\
\cdots\end{array}$ \\
\hline 77669 & 7.70766 & $5 \mathrm{e}-04$ & 0.180 & 0.15 & $\cdots$ & 8.10 & References \\
$\cdots$ & B9III/IV & G5II/III+A3 \\
142049 & 13.22062 & $4 \mathrm{e}-05$ & 0.050 & 0.045 & 45942 & 5.85 & 1 \\
155781 & 13.08670 & $6 \mathrm{e}-05$ & 0.100 & 0.080 & $\cdots$ & 7.42 & A3IV/V & 2 \\
\hline
\end{tabular}

References. (1) Houk (1978), (2) Houk \& Cowley (1975).

Table 4

Eclipsing Binaries Showing the O'Connell Effect Detected with bRing

\begin{tabular}{lccccr}
\hline \hline Name & $\begin{array}{c}A \\
\ldots\end{array}$ & $\begin{array}{c}\sigma_{A} \\
(\mathrm{mmag})\end{array}$ & $\begin{array}{c}\text { Max 1 } \\
(\mathrm{mag})\end{array}$ & $\begin{array}{c}\text { Max 2 } \\
(\mathrm{mag})\end{array}$ & $\begin{array}{r}\Delta m \\
(\mathrm{mmag})\end{array}$ \\
\hline del Pic & 46.9 & 0.6 & 4.6275 & 4.6301 & 2.6 \\
V462 Car & 30.7 & 0.4 & 6.5196 & 6.5120 & 7.6 \\
TY Men & 99.0 & 1.0 & 8.0567 & 8.0925 & 35.8 \\
V535 Ara & 30.2 & 0.4 & 7.3529 & 7.3551 & 2.2 \\
V954 Sco & 48.9 & 0.8 & 7.4828 & 7.4933 & 10.5 \\
V772 Car & 27.4 & 0.4 & 8.0544 & 8.0580 & 3.6 \\
QZ Pup & 8.4 & 0.2 & 4.4553 & 4.4584 & 3.1 \\
DE Mic & 16.6 & 0.5 & 7.7230 & 7.7323 & 9.3 \\
HD 123720 & 16.4 & 0.3 & 7.7724 & 7.7772 & 4.8 \\
LZ Cen & 88.6 & 1.2 & 8.3148 & 8.3288 & 14.0 \\
V979 Cen & 18.0 & 0.3 & 7.4619 & 7.4654 & 3.5 \\
V1012 Sco & 12.0 & 0.2 & 6.6502 & 6.6525 & 2.3 \\
V716 Cen & 32.9 & 0.5 & 6.0115 & 6.0072 & 4.3 \\
QX Vel & 37.8 & 0.6 & 7.8886 & 7.8824 & 6.2 \\
HR 6247 & 44.8 & 0.8 & 2.8767 & 2.8705 & 6.2 \\
V470 Car & 19.9 & 0.4 & 7.2323 & 7.2298 & 2.5 \\
TU Mus & 101.9 & 1.3 & 8.2469 & 8.2266 & 20.4 \\
RR Cen & 71.8 & 1.1 & 7.2808 & 7.2752 & 5.6 \\
\hline
\end{tabular}

were recently published and analyzed in Zwintz et al. (2019). The mismatches may be due to a variety of reasons, including aliasing or the presence of multiple modes; however, the star $\theta$ Tuc had an additional feature in its periodogram that is not $\delta$ Scuti in nature.

HD $3112(\theta$ Tuc $): \theta$ Tuc is a well-studied $\delta$ Scuti that is also a well-studied binary system (e.g., Cousins \& Lagerweij 1971; Stobie \& Shobbrook 1976; Kurtz 1980; Bos 1994; Sterken 1997; De Mey et al. 1998). The primary pulsation reported in the VSX catalog is 20.28068 day $^{-1}$, which agrees with prior observations (Cousins \& Lagerweij 1971; Stobie \& Shobbrook 1976; Kurtz 1980; Liakos \& Niarchos 2017). However, the dominant period detected by bRing is 0.28165 day $^{-1}$, which is reported in Table 5. This pulsation has been previously identified as orbital motion associated with the binary nature of the system (0.281 day ${ }^{-1}$ : Sterken 1997; De Mey et al. 1998). A search of the bRing periodogram around the expected $\delta$ Scuti frequencies recovers a primary $\delta$ Scuti frequency of 17.06312 day $^{-1}$.

The 26 new candidate $\delta$ Scuti variables all had faint primary pulsation amplitudes of $<10.5 \mathrm{mmag}$, with the exception of HD 216743, and they all had brightnesses in the range $V \simeq 6.5-8.3$, and are reported in Table 6 (showing the primary pulsation frequency as seen by bRing). Most of the newly discovered $\delta$ Scuti variables in the bRing survey were in the faint end of the magnitude range for the instrument $(V<6.5)$; the two brighter candidates were HD $171819(V=5.84)$ and HD 189951 ( $V=5.25$ ) (Kharchenko \& Roeser 2009). Further analysis of the frequencies detected with the bRing time-series photometry for the previously discovered and newly discovered $\delta$ Scuti variables is encouraged and out of the scope of this work.

The star HD 140566 (included in Table 7) was labeled as detached EB by the VSX with a period 193.70000 days. bRing detected a much shorter period at 0.08783 days $(11.38563$ day ${ }^{-1}$ ). This star is poorly studied with no prior follow-up work attempting to confirm the nature of this variable. The bRing period and light curve are not indicative of an eclipsing system. The combination of the detected pulsation in the bRing light curve and the star's spectral type (A5IV) indicate that the star is likely to be a $\delta$ Scuti variable. This agrees with its position among other $\delta$ Scutis in the CMD from Figure 4 $\left((B-V)_{0}=0.16, M_{V}=0.99\right)$. Therefore, this star is not an eclipsing system and is reclassified in this work as a candidate $\delta$ Scuti.

\subsection{Other Variables}

In addition to the variables discussed in the previous sections, bRing detected evidence of periodic pulsations representing several different "other" classes of variability including ellipsoidal variables (ELLs), rotation periods (ROT), and $\beta$ Cepheids (BCEPs), among others. bRing was particularly sensitive to low-amplitude (typically $\simeq 10 \mathrm{mmag}$ ) slowly pulsating B stars (SPBs) and LPVs. In Table 7, we list 80 stars previously classified as variables in the VSX catalog, along with their VSX and bRing variability parameters, and classification in the VSX catalog (last column). We discuss some of these stars further in Section 4.4.1 if the period of a non-LPV star detected with bRing was significantly different than a previously published period or if the periodogram revealed new additional periods of interest. In Table 8, a list of new period detections or variable classifications is provided for 25 stars using this system based on their spectral and pulsation properties and light-curve shapes, and these stars are discussed further in Section 4.4.2.

\subsubsection{Previously Known Variables}

In comparing the periods determined using bRing data to those listed in either VSX or Rimoldini et al. (2012), we find that only 20 of the $80(25 \%)$ had completely different periods. We discuss the ones that showed period differences in this section.

bRing was able to provide more precise period measurements for five of the LPV, SR, and SRD stars. Five of these stars had completely different periods from the low-precision periods reported in VSX or Rimoldini et al. (2012).

HD 177171 ( $\rho \mathrm{Tel}$ ): for the young F5V HD 177171, we detect a strong periodicity of 1.55258 days. However, Rimoldini et al. (2012) quote a period of 0.71187 day, and VSX reports a period of 4.73687 days from Koen \& Eyer (2002). Both estimates are based on the sparse Hipparcos time-series photometry ( $\sim 70$ data points), 

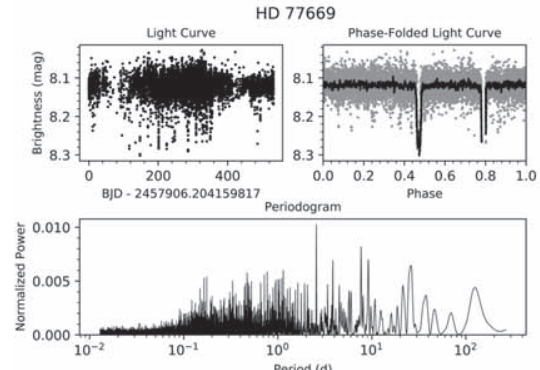

(a)
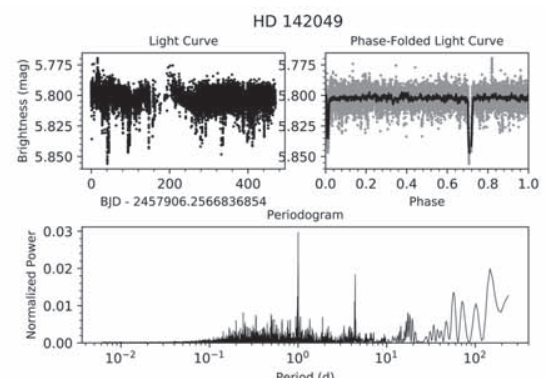

(b)
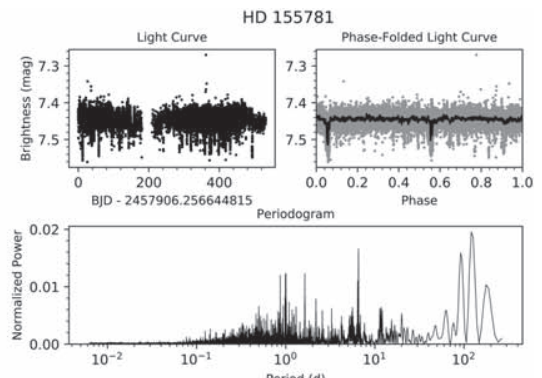

(c)

Figure 5. Light curves, periodograms, and phase-folded light curves of the three new eclipsing binaries detected in this survey.

Table 5

Previously Classified $\delta$ Scutis Detected with bRing

\begin{tabular}{|c|c|c|c|c|c|c|c|c|c|c|}
\hline $\begin{array}{l}\text { Name } \\
\ldots\end{array}$ & $\begin{array}{c}\mathrm{HD} \\
\ldots\end{array}$ & $\begin{array}{c}f \\
\left(\text { day }^{-1}\right)\end{array}$ & $\begin{array}{c}\sigma_{f} \\
\left(\text { day }^{-1}\right)\end{array}$ & $\begin{array}{c}A \\
(\mathrm{mmag})\end{array}$ & $\begin{array}{c}\sigma_{A} \\
(\mathrm{mmag})\end{array}$ & $\begin{array}{c}\text { VSX ID } \\
\ldots\end{array}$ & $\begin{array}{c}f_{\mathrm{VSX}} \\
\left(\mathrm{day}^{-1}\right)\end{array}$ & $\begin{array}{c}V \\
(\mathrm{mag})\end{array}$ & $\mathrm{SpT}$ & $\begin{array}{c}\text { References } \\
\ldots\end{array}$ \\
\hline$\theta$ Tuc & 3112 & 0.28165 & $2 \mathrm{e}-04$ & 4.0 & 0.2 & 37102 & 20.28068 & 6.11 & kA7hA7mF0(IV) & 1 \\
\hline$\ldots$ & 8351 & 14.13144 & $5 e-05$ & 3.7 & 0.2 & 53727 & $14.06695^{\mathrm{a}}$ & 6.70 & A9V & 2 \\
\hline BD Phe & 11413 & 27.02703 & $4 \mathrm{e}-03$ & 4.9 & 0.2 & 26294 & $25.21158^{\mathrm{a}}$ & 5.93 & $\mathrm{~A} 1 \mathrm{Va} \lambda \mathrm{Boo}$ & 3 \\
\hline$\ldots$ & 12284 & 6.25500 & $7 e-05$ & 5.2 & 0.3 & 53855 & 6.20694 & 7.68 & A9III & 2 \\
\hline RX Cae & 28837 & 8.27307 & $5 e-04$ & 6.5 & 0.3 & 4527 & 6.48925 & 7.01 & F3/F5II & 4 \\
\hline $\mathrm{X} \mathrm{Cae}$ & 32846 & 0.29564 & $1 \mathrm{e}-04$ & 5.0 & 0.3 & 4518 & 0.27049 & 6.31 & $\mathrm{~F} 2 \mathrm{IV} / \mathrm{V}$ & 2 \\
\hline YY Pic & 39244 & 18.51316 & $3 e-04$ & 3.7 & 0.2 & 26385 & 9.73985 & 7.79 & A7V & 4 \\
\hline$\cdots$ & 41846 & 10.47770 & $4 e-05$ & 5.3 & 0.2 & $\ldots$ & $10.33475^{\mathrm{a}}$ & 8.12 & A6mA7-F0 & 5 \\
\hline$\ldots$ & 46586 & 14.82017 & $5 e-04$ & 2.9 & 0.2 & 410727 & 14.82052 & 8.04 & FOIII & 4 \\
\hline V638 Pup & 58635 & 8.66695 & $5 e-03$ & 2.5 & 0.1 & 26970 & 8.66699 & 6.82 & $\mathrm{~A} 8 \mathrm{~V}$ & 2 \\
\hline V393 Car & 66260 & 14.1551 & $1 \mathrm{e}-04$ & 6.8 & 0.2 & 6146 & 7.07741 & 7.47 & A7III/IV & 5 \\
\hline AI Vel & 69213 & 11.59990 & $1 \mathrm{e}-04$ & 49.0 & 0.9 & 37479 & 8.96265 & 6.56 & A9IV-V & 4 \\
\hline OX Vel & 77347 & 12.60254 & $1 \mathrm{e}-04$ & 11.5 & 0.2 & 37727 & 12.60255 & 7.58 & A4mA7-A9 & 5 \\
\hline ER Cha & 88278 & 14.27857 & $1 \mathrm{e}-04$ & 3.9 & 0.1 & 9398 & 15.72376 & 7.31 & A3/5III/IV & 5 \\
\hline LW Vel & 88824 & 12.58582 & $5 \mathrm{e}-05$ & 3.8 & 0.1 & 37687 & 8.98093 & 5.27 & F0Vn & 1 \\
\hline$\cdots$ & 90611 & 15.19447 & $4 \mathrm{e}-04$ & 2.5 & 0.2 & $\ldots$ & $15.19498^{\mathrm{a}}$ & 6.55 & FOIV/V & 4 \\
\hline IW Vel & 94985 & 10.14809 & $2 \mathrm{e}-04$ & 4.3 & 0.1 & 37656 & 6.66666 & 5.90 & $\mathrm{~A} 4 \mathrm{~V}$ & 4 \\
\hline V1023 Cen & 102541 & 19.89813 & $5 e-03$ & 4.1 & 0.2 & 8320 & $20.00000^{\mathrm{b}}$ & 7.95 & hF0VkA5mA5 $\lambda$ Boo & 6 \\
\hline EE Cha & 104036 & 33.86956 & $3 e-04$ & 3.4 & 0.1 & 9386 & $33.33333^{\mathrm{b}}$ & 6.73 & A7V & 5 \\
\hline$\ldots$ & 111984 & 23.49741 & $5 e-05$ & 3.3 & 0.1 & $\ldots$ & $21.46347^{\mathrm{a}}$ & 7.28 & A5V & 4 \\
\hline V853 Cen & 126859 & 16.30857 & $8 \mathrm{e}-04$ & 2.4 & 0.3 & 8150 & 18.92013 & 6.97 & A6V & 5 \\
\hline IN Lup & 142994 & 9.16564 & $6 e-05$ & 3.0 & 0.2 & 17824 & $7.87402^{\mathrm{b}}$ & 7.17 & F2VkA3mA3 $\lambda$ Boo? & 7 \\
\hline IO Lup & 143232 & 13.40241 & $5 e-04$ & 3.0 & 0.1 & 17825 & $15.59193^{\mathrm{a}}$ & 6.67 & kA7hA5mF2 & 8 \\
\hline V922 Sco & 153747 & 23.80734 & $1 \mathrm{e}-03$ & 2.7 & 0.2 & 33738 & 20.00000 & 7.40 & hA7VmA0 $\lambda$ Boo & 6 \\
\hline$\ldots$ & 156623 & 71.14754 & $8 \mathrm{e}-04$ & 3.4 & 0.1 & $\ldots$ & $71.14300^{c}$ & 7.24 & A1V PHL & 6 \\
\hline$\ldots$ & 157321 & 10.51587 & $2 \mathrm{e}-05$ & 9.7 & 0.2 & 60284 & 10.51640 & 8.02 & A9IV/V & 5 \\
\hline V703 Sco & 160589 & 6.66876 & $6 e-04$ & 25.2 & 1.4 & 33519 & 8.67922 & 7.85 & F0V & 6 \\
\hline V346 Pav & 168740 & 12.57296 & $1 \mathrm{e}-04$ & 2.6 & 0.1 & 25066 & $16.98244^{\mathrm{d}}$ & 6.12 & $\mathrm{~A} 8 \mathrm{VkA} 2 \mathrm{~mA} 2 \lambda$ Boo & 7 \\
\hline V353 Tel & 173794 & 0.61578 & $3 e-04$ & 4.7 & 0.1 & 137348 & 0.31250 & 7.11 & A3III/IV & 4 \\
\hline QQ Tel & 185139 & 8.41297 & $3 e-04$ & 4.3 & 0.1 & 36568 & 15.38462 & 6.26 & F2IV & 4 \\
\hline$\cdots$ & 192316 & 33.0221 & $1 \mathrm{e}-03$ & 3.4 & 0.2 & $\ldots$ & $22.80867^{\mathrm{a}}$ & 7.55 & $\mathrm{~A} 8 \mathrm{~V}$ & 5 \\
\hline$\cdots$ & 198592 & 26.34244 & $8 \mathrm{e}-04$ & 2.7 & 0.3 & $\cdots$ & $21.59235^{\mathrm{a}}$ & 7.58 & A3III & 4 \\
\hline$\ldots$ & 200475 & 1.11423 & $2 \mathrm{e}-05$ & 9.5 & 0.2 & 305774 & 1.11499 & 7.82 & A3mA5-A7 & 5 \\
\hline$\cdots$ & 201292 & 14.7769 & $4 \mathrm{e}-05$ & 5.2 & 0.2 & $\ldots$ & $25.80477^{\mathrm{a}}$ & 8.19 & A3II & 5 \\
\hline CK Ind & 209295 & 1.12963 & $3 e-04$ & 25.3 & 0.3 & 137616 & 1.12934 & 7.32 & A9/F0V & 5 \\
\hline DR Gru & 213669 & 14.01768 & $7 e-05$ & 7.6 & 0.2 & 137628 & 15.01502 & 7.41 & F0VkA2.5mA2.5 $\lambda$ Boo & 7 \\
\hline$\cdots$ & 218090 & 1.81606 & $1 \mathrm{e}-04$ & 6.3 & 0.1 & 305852 & 1.81590 & 8.13 & F0V & 5 \\
\hline$\ldots$ & 219301 & 9.28928 & $3 e-05$ & 3.6 & 0.2 & 250337 & $9.28966^{\mathrm{a}}$ & 6.56 & FOIII & 5 \\
\hline RS Gru & $\cdots$ & 13.60412 & $6 e-05$ & 46.9 & 0.7 & 14680 & 6.80218 & 8.27 & A9IV & 4 \\
\hline HIP 35815 & $\ldots$ & 7.70897 & $5 e-05$ & 6.9 & 0.3 & 55889 & $10.83677^{\mathrm{a}}$ & 7.84 & F0 & 9 \\
\hline
\end{tabular}

Notes.

${ }^{\text {a }}$ Rimoldini et al. (2012).

${ }^{b}$ Rodríguez et al. (2000).

c Mellon et al. (2019a).

d Paunzen et al. (1998).

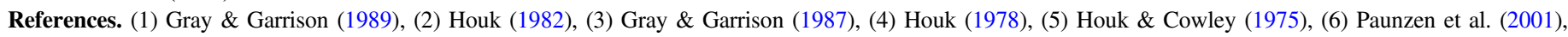
(7) Gray et al. (2017), (8) Paunzen \& Duffee (1996), (9) Boss (1937). 
Table 6

New Candidate $\delta$ Scutis Detected with bRing

\begin{tabular}{|c|c|c|c|c|c|c|c|c|}
\hline $\begin{array}{l}\mathrm{HD} \\
\quad \ldots\end{array}$ & $\begin{array}{c}f \\
\left(\text { day }^{-1}\right)\end{array}$ & $\begin{array}{c}\sigma_{f} \\
\left(\mathrm{day}^{-1}\right)\end{array}$ & $\begin{array}{c}A \\
\text { (mmag) }\end{array}$ & $\begin{array}{c}\sigma_{A} \\
(\mathrm{mmag})\end{array}$ & $\begin{array}{c}\text { VSX ID } \\
\ldots\end{array}$ & $\begin{array}{c}V \\
(\mathrm{mag})\end{array}$ & $\begin{array}{l}\text { SpT } \\
\cdots\end{array}$ & $\begin{array}{c}\text { References } \\
\ldots\end{array}$ \\
\hline 3463 & 10.50785 & $5 e-04$ & 3.7 & 0.2 & $\cdots$ & 8.04 & A6/8V & 1 \\
\hline 20232 & 45.45826 & $2 \mathrm{e}-04$ & 2.1 & 0.2 & $\cdots$ & 6.88 & A2/A3III/IV & 2 \\
\hline 25860 & 15.34882 & $4 e-04$ & 2.0 & 0.1 & $\cdots$ & 6.62 & A4/A5IV & 1 \\
\hline 43898 & 19.31816 & $2 \mathrm{e}-04$ & 2.6 & 0.3 & $\cdots$ & 7.87 & A8/A9V & 2 \\
\hline 46978 & 16.6692 & $2 e-03$ & 4.4 & 0.2 & $\ldots$ & 8.16 & A6V & 1 \\
\hline 57969 & 68.46621 & $5 e-03$ & 2.1 & 0.1 & 55878 & 6.56 & A1V & 1 \\
\hline 72979 & 10.49512 & $8 e-04$ & 6.0 & 0.1 & $\cdots$ & 7.70 & A4Vs & 1 \\
\hline 81771 & 11.81314 & $4 \mathrm{e}-04$ & 3.6 & 0.1 & $\ldots$ & 7.76 & $\mathrm{~A} 4 \mathrm{~V}$ & 1 \\
\hline 82484 & 4.53561 & $8 \mathrm{e}-03$ & 7.2 & 0.3 & $\ldots$ & 8.09 & A3III/IV & 2 \\
\hline 92762 & 16.39493 & $8 \mathrm{e}-04$ & 4.1 & 0.2 & $\ldots$ & 7.80 & A8V & 1 \\
\hline 110080 & 18.55808 & $6 e-04$ & 2.7 & 0.1 & $\ldots$ & 7.41 & $\mathrm{~A} 5 \mathrm{~V}$ & 1 \\
\hline 121191 & 21.59957 & $3 e-03$ & 7.7 & 0.2 & $\ldots$ & 8.16 & A5IV/V & 3 \\
\hline 156408 & 10.64459 & $3 e-04$ & 10.4 & 0.3 & 33894 & 8.27 & A7V & 2 \\
\hline 163482 & 5.92257 & $3 e-03$ & 3.0 & 0.1 & 274692 & 6.82 & A0III/IV & 2 \\
\hline 168651 & 15.66042 & $3 e-04$ & 2.5 & 0.2 & $\cdots$ & 7.40 & A9III & 3 \\
\hline 170461 & 12.59065 & $5 e-04$ & 3.7 & 0.2 & 250253 & 6.98 & A9IV & 2 \\
\hline 171819 & 13.55119 & $2 e-04$ & 2.4 & 0.3 & $\cdots$ & 5.84 & $\mathrm{~A} 7 \mathrm{IV} / \mathrm{V}$ & 3 \\
\hline 172995 & 13.17514 & $2 e-04$ & 3.3 & 0.1 & $\cdots$ & 6.81 & A9IV & 3 \\
\hline 177523 & 13.11519 & $2 \mathrm{e}-04$ & 2.6 & 0.2 & $\cdots$ & 7.49 & A9/F0IV & 3 \\
\hline 177665 & 11.34302 & $2 \mathrm{e}-04$ & 5.1 & 0.2 & $\cdots$ & 8.37 & F2IV & 3 \\
\hline 189951 & 12.19356 & $5 e-04$ & 2.1 & 0.3 & 63419 & 5.25 & A9IV & 3 \\
\hline 191585 & 15.46432 & $4 e-04$ & 4.4 & 0.1 & $\cdots$ & 6.92 & A2/3IV & 2 \\
\hline 200203 & 19.31605 & $7 e-04$ & 2.1 & 0.3 & $\cdots$ & 7.35 & A4/A5II/III & 1 \\
\hline 204352 & 14.35455 & $3 e-05$ & 2.9 & 0.2 & $\cdots$ & 8.40 & A9V & 1 \\
\hline 208094 & 18.46880 & $4 e-04$ & 3.0 & 0.3 & $\cdots$ & 8.21 & A2IV & 1 \\
\hline 216743 & 16.88458 & $3 e-04$ & 43.3 & 0.2 & $\cdots$ & 7.25 & A3V & 3 \\
\hline
\end{tabular}

References. (1) Houk \& Cowley (1975), (2) Houk (1982), (3) Houk (1978).

whereas the bRing data set has $>10^{2}$ more points and dense coverage. We do not detect significant peaks at the periods reported by either Rimoldini et al. (2012) or Koen \& Eyer (2002) (in VSX).

HD 60168 (PS Pup): with bRing we detected a slightly different period (2.07742 days) for the ELL variable PS Pup compared to that published in VSX (1.34220 days). The periodogram for PS Pup does not have a significant period near the VSX period of 1.34220 days and the detected bRing period is near the 2 day alias of the sidereal systematic. The star and its periodicity detected with bRing are reported in Table 7 because the expected shape of an ELL variable is recovered at this period versus the sinusoid expected from a sidereal alias (hence we believe the periodicity to be real).

HD 172416 and HD 189631: the $\gamma$ Dor-type stars HD 172416 and HD 189631 both had different periods in bRing than they have reported in VSX. For HD 172416, the reported VSX period is 0.99787 day, which is close to the primary sidereal systematic. If this was a real signal in bRing, it could not be recovered due to the proximity to this dominant systematic. The 0.59900 day signal for HD 172416 was not recovered at all in the bRing periodogram and the detrending routine should not affect this period. The periodogram for this star revealed the primary period of 0.70578 day, as well as additional significant periods that could be useful for future analysis.

V946 Cen, HD 116862, and V846 Ara: there were several Be stars detected by bRing. bRing measured a different period for the stars V946 Cen, HD 116862, and V846 Ara compared to that reported in VSX; these three stars also happen to be $\gamma$ Cas (GCAS) variables, which are known to be irregularly variable on the order of a decade. The time between the study of Rimoldini et al. (2012) and bRing ( 5-7 yr) could be enough time for periods to drift, causing the differences in observed periods. bRing's long baseline could also be a factor, picking up underlying shifts. In particular, V946 Cen and HD 116862 both show two dominant periods, while V846 Ara only shows a single dominant period at 0.43861 day.

The RS CVn (RS) variables hosted the most discrepancies between observed bRing periods and prior studies. bRing detected completely different periods for $\rho$ Tel, HD 201247, and HD 209234; bRing detected near half the original period for HD 56142.

bRing detected 26 slowly pulsating B-type (SPB) stars (including 4 new ones from Section 4.4.2 and the reclassified classical Cepheid HD 136633 from Section 4.1). These stars are characterized by their spectral type, location near the mainsequence (as observed for this sample in Figure 4), and periods ranging from just short of a day to several days (De Cat 2007; Miglio et al. 2007). They are also known to exhibit multiple oscillations in their light curves (Miglio et al. 2007) and have even coexhibited BCEP pulsations in a few rare cases (De Cat 2007).

The periodograms for all of the SPB variables were individually inspected for multiple periods or shorter periods that may indicate BCEP pulsations similar to the stars from (De Cat 2007). In general, the previously observed SPB variables showed multiple independent periods in their periodograms and bRing detected different primary periods for HD 85871 and HD 159041. Further analysis of the SPB stars detected with bRing is beyond the scope of this work. 
Table 7

Other Previously Classified Variables Detected with bRing

\begin{tabular}{|c|c|c|c|c|c|c|c|c|c|c|c|}
\hline $\begin{array}{r}\text { Name } \\
\ldots\end{array}$ & $\begin{array}{c}\mathrm{HD} \\
\ldots\end{array}$ & $\begin{array}{c}P \\
\text { (day) }\end{array}$ & $\begin{array}{c}\sigma_{P} \\
\text { (day) }\end{array}$ & $\begin{array}{c}A \\
(\mathrm{mmag})\end{array}$ & $\begin{array}{c}\sigma_{A} \\
(\mathrm{mmag})\end{array}$ & $\begin{array}{c}\text { VSX ID } \\
\ldots\end{array}$ & $\begin{array}{l}P_{\mathrm{VSx}} \\
\text { (day) }\end{array}$ & $\begin{array}{c}V \\
(\mathrm{mag})\end{array}$ & $\begin{array}{l}\mathrm{SpT} \\
\ldots\end{array}$ & $\begin{array}{c}\text { References } \\
\ldots\end{array}$ & $\begin{array}{l}\text { Type } \\
\text { (VSX) }\end{array}$ \\
\hline$\ldots$ & 13397 & 48.32756 & $4 \mathrm{e}-02$ & 10 & 0.2 & 280885 & 49.20000 & 7.74 & K0III & 1 & ROT \\
\hline UX For & 17084 & 0.95197 & $1 \mathrm{e}-03$ & 21.3 & 0.5 & 14260 & 0.95600 & 8.04 & $\mathrm{G} 5 / 8 \mathrm{~V}+(\mathrm{G})$ & 2 & RS \\
\hline TV Pic & 30861 & 0.85175 & $3 e-05$ & 30.1 & 0.4 & 26362 & 0.85199 & 7.44 & $\mathrm{~A} 2 \mathrm{~V}$ & 1 & ELL \\
\hline TU Pic & 33331 & 1.14708 & $2 \mathrm{e}-04$ & 9.0 & 0.2 & 26361 & 1.14686 & 6.90 & B5III & 1 & SPB \\
\hline R Pic & 30551 & 141.89257 & $26 \mathrm{e} 00$ & 523.7 & 5.4 & 26334 & 168.00000 & 7.59 & K2/K3II:pe & 1 & SR \\
\hline YZ Men & 34802 & 19.36778 & $8 \mathrm{e}-02$ & 24.1 & 0.3 & 18686 & 19.58000 & 7.76 & K1IIIp & 3 & RS \\
\hline AB Dor & 36705 & 0.51443 & $2 \mathrm{e}-04$ & 10.6 & 0.2 & 13645 & 0.513900 & 6.93 & K1III(p) & 3 & TTS/ROT \\
\hline lam Col & 39764 & 1.28660 & $1 \mathrm{e}-03$ & 1.4 & 0.3 & 9602 & 1.28701 & 4.87 & $\mathrm{~B} 5 \mathrm{~V}$ & 2 & ELL \\
\hline SZ Pic & 39917 & 4.94895 & $4 \mathrm{e}-03$ & 0.1 & 0.4 & 26359 & 4.95000 & 7.89 & G8V & 2 & ELL \\
\hline TY Pic & 42504 & 48.82267 & $3 e-01$ & 7.8 & 0.3 & 26365 & 50.20000 & 7.70 & G8/K0III+F & 3 & RS \\
\hline V Pic & 43518 & 166.32289 & $2 \mathrm{e} 00$ & 16.8 & 0.2 & 26338 & 180.00000 & 7.41 & K2III & 3 & SR \\
\hline AE Men & 46291 & 12.14125 & $2 \mathrm{e}-02$ & 13.9 & 0.2 & 18692 & 12.03000 & 8.25 & $\mathrm{~K} 2 \mathrm{III}+\mathrm{F} / \mathrm{G}$ & 3 & $\mathrm{RS}$ \\
\hline TZ Pic & 46697 & 13.64700 & $3 e-03$ & 13.7 & 0.2 & 26366 & 13.68000 & 7.64 & $\mathrm{~K} 1 \mathrm{III} / \mathrm{IVp}$ & 3 & $\mathrm{RS}$ \\
\hline V448 Car & 49877 & 55.99252 & $1 \mathrm{e}-01$ & 49.8 & 0.5 & 6201 & $\ldots$ & 5.61 & K5III & 3 & SRD \\
\hline$\ldots$ & 56142 & 10.57968 & $4 \mathrm{e}-01$ & 6.1 & 0.3 & 55829 & 21.16000 & 7.57 & F6/F7V & 2 & RS \\
\hline PS Pup & 60168 & 2.07742 & $2 \mathrm{e}-03$ & 8.1 & 0.4 & 26919 & 1.34220 & 6.62 & B8V & 2 & ELL \\
\hline V372 Car & 64722 & 0.11540 & $1 \mathrm{e}-05$ & 4.6 & 0.1 & 6125 & 0.11600 & 5.68 & B1.5IV & 3 & BCEP \\
\hline V413 Pup & 66235 & 1.59433 & $9 \mathrm{e}-04$ & 11.1 & 0.3 & 27015 & 1.59406 & 7.68 & B9IV & 1 & SPB \\
\hline V415 Pup & 66503 & 0.84905 & $1 e-04$ & 7.5 & 0.2 & 27017 & 0.84892 & 8.22 & B5V & 1 & SPB \\
\hline QR Pup & 69342 & 3.55155 & $7 \mathrm{e}-04$ & 21.5 & 0.4 & 26928 & 3.55180 & 8.06 & B3II & 1 & ELL \\
\hline HV Vel & 73340 & 2.66807 & $8 \mathrm{e}-04$ & 6.2 & 0.1 & 37638 & 2.66745 & 5.78 & $\mathrm{ApSi}$ & 1 & roAp \\
\hline omi Vel & 74195 & 2.79716 & $1 \mathrm{e}-03$ & 7.3 & 0.2 & 37807 & 2.79759 & 3.59 & B3IV & 1 & SPB \\
\hline$\cdots$ & 74422 & 0.74500 & 0.001 & 42.4 & 0.5 & 400557 & 0.74500 & 8.12 & A3IV & 3 & ACEP \\
\hline V473 Car & 76640 & 0.95421 & $1 \mathrm{e}-04$ & 8.1 & 0.1 & 6226 & 0.95399 & 6.35 & B5V & 3 & SPB \\
\hline OW Vel & 76875 & 66.50249 & $2 \mathrm{e}+00$ & 51.7 & 0.6 & 37726 & 64.54000 & 7.66 & $\mathrm{~K} 2 / 3 \mathrm{III}+\mathrm{A} / \mathrm{F}$ & 1 & SRD \\
\hline OY Vel & 77653 & 1.48775 & $4 \mathrm{e}-04$ & 6.6 & 0.1 & 37728 & 1.48782 & 5.03 & B9 & 1 & $\mathrm{ACV}$ \\
\hline PR Vel & 78405 & 1.23898 & $4 \mathrm{e}-04$ & 10.6 & 0.3 & 37732 & 1.23890 & 8.26 & B5IV & 1 & SPB \\
\hline PS Vel & 79039 & 1.07481 & 1e-04 & 9.4 & 0.2 & 37733 & 1.07460 & 6.82 & B4V & 1 & SPB \\
\hline V480 Car & 81654 & 40.80297 & $5 \mathrm{e}-01$ & 18.9 & 0.5 & 6233 & $40.00369^{\mathrm{a}}$ & 7.87 & $\mathrm{~B} 2 / 3 \mathrm{~V}(\mathrm{e})$ & 3 & $\mathrm{BE}+\mathrm{GCAS}$ \\
\hline QZ Vel & 85871 & 5.71790 & $7 \mathrm{e}-03$ & 5.2 & 0.2 & 37750 & 1.03108 & 6.49 & B1V & 3 & SPB \\
\hline V335 Vel & 85953 & 3.75717 & $3 e-03$ & 6.1 & 0.1 & 37751 & 3.75520 & 5.94 & $\mathrm{~B} 2 \mathrm{~V}$ & 4 & SPB \\
\hline$\cdots$ & 88825 & 1.45753 & $3 e-05$ & 10.5 & 0.2 & $\cdots$ & $\cdots$ & 6.09 & B4Ve & 3 & BE(SPB) \\
\hline V514 Car & 92287 & 2.90561 & $6 e-04$ & 3.8 & 0.1 & 6267 & 2.90457 & 5.88 & B3IV & 3 & ELL \\
\hline V431 Car & 97152 & 1.61853 & $1 \mathrm{e}-05$ & 6.7 & 0.2 & 6184 & 1.61853 & 8.07 & $\mathrm{WC} 7+\mathrm{O} 7 \mathrm{~V}$ & 5 & E/WR \\
\hline KQ Mus & 100359 & 1.23848 & $3 e-04$ & 13.6 & 0.2 & 19926 & 1.23834 & 6.88 & B7IV & 3 & SPB \\
\hline V810 Cen & 101947 & 151.28362 & $7 e-01$ & 16.0 & 0.2 & 8107 & 130.00000 & 5.01 & F9Ia & 6 & LPV \\
\hline DE Cru & 104631 & 3.68406 & $3 e-03$ & 10.2 & 0.2 & 10896 & 3.68800 & 6.77 & B1II & 3 & SPB \\
\hline DF Cru & 104705 & 1.13486 & $2 \mathrm{e}-04$ & 13.8 & 0.2 & 10897 & 1.13480 & 7.81 & B0.5III & 3 & SPB \\
\hline V1123 Cen & 108015 & 58.59871 & $1 e+00$ & 42.5 & 0.6 & 44225 & 60.60000 & 7.97 & $\mathrm{~F} 3 / 5 \mathrm{Ib} / \mathrm{II}$ & 1 & SRD \\
\hline V946 Cen & 112999 & 1.13651 & $1 \mathrm{e}-04$ & 12.2 & 0.2 & 8243 & $0.08883^{\mathrm{a}}$ & 7.38 & B6III(n) & 7 & $\mathrm{BE}+\mathrm{GCAS}$ \\
\hline$\ldots$ & 116862 & 0.80112 & $7 \mathrm{e}-04$ & 3.7 & 0.1 & 58241 & $2.87078^{\mathrm{a}}$ & 6.26 & B3IV & 1 & $\mathrm{BE}+\mathrm{GCAS}$ \\
\hline$\cdots$ & 118258 & 49.69547 & $1 \mathrm{e}+00$ & 4.8 & 0.2 & 287154 & 50.50000 & 8.01 & G6V & 3 & $\mathrm{RS}$ \\
\hline DF Cir & 124672 & 0.36907 & $1 \mathrm{e}-05$ & 13.7 & 0.2 & 136640 & 0.367772 & 7.55 & F6V & 8 & ELL \\
\hline V1001 Cen & 125104 & 6.73825 & $1 \mathrm{e}-02$ & 10.1 & 0.3 & 8298 & 6.73600 & 7.29 & B4IV/V & 3 & DPV/ELL \\
\hline HX Lup & 125721 & 3.08809 & $7 e-04$ & 5.0 & 0.1 & 17817 & 3.08809 & 6.11 & B1III & 1 & ELL \\
\hline V761 Cen & 125823 & 8.81327 & $2 \mathrm{e}-02$ & 6.5 & 0.3 & 8058 & 8.81710 & 4.41 & $\mathrm{~B} 2 \mathrm{~V}$ & 2 & SXARI \\
\hline eta Cen & 127972 & 0.64250 & $6 e-05$ & 7.9 & 0.3 & 8347 & 0.64247 & 2.33 & $\mathrm{~B} 1 \mathrm{Vn}+\mathrm{A}$ & 9 & GCAS+LERI \\
\hline LS TrA & 137164 & 44.46069 & $6 e-01$ & 39.9 & 0.4 & 36931 & 45.00000 & 7.47 & $\mathrm{~K} 1 / \mathrm{K} 2 \mathrm{IVp}$ & 3 & RS \\
\hline HV Lup & 137518 & 2.82838 & $2 \mathrm{e}-03$ & 38.9 & 0.7 & 17815 & $\cdots$ & 7.74 & B1/2(I/IIIN) & 9 & $\mathrm{BE}$ \\
\hline LZ TrA & 138521 & 0.57015 & $6 e-05$ & 7.4 & 0.2 & 36938 & 0.57019 & 8.04 & B9IV & 3 & SPB \\
\hline$\ldots$ & $140566^{\mathrm{b}}$ & 0.08783 & $1 \mathrm{e}-05$ & 3.3 & 0.2 & 415978 & 193.70000 & 8.28 & A5IV & 1 & ESD(DSCT) \\
\hline$\cdots$ & 142542 & 92.14955 & $2 \mathrm{e}+00$ & 4.1 & 0.2 & 412695 & 324.00000 & 6.29 & F3/F5V & 2 & M \\
\hline V374 Nor & 147894 & 2.77589 & $3 e-04$ & 6.7 & 0.2 & 20334 & 2.72950 & 7.24 & B5III & 1 & ELL \\
\hline V918 Sco & 149404 & 9.81300 & $5 e-03$ & 4.8 & 0.1 & 33734 & 9.81300 & 5.48 & O9Ia & 10 & ELL \\
\hline$\ldots$ & 149455 & 1.28303 & $2 \mathrm{e}-04$ & 6.8 & 0.2 & 59146 & 1.28217 & 7.69 & B7III/IV & 3 & SPB \\
\hline$\cdots$ & 151158 & 0.18178 & $1 \mathrm{e}-05$ & 6.3 & 0.2 & 225575 & 0.18178 & 8.21 & $\mathrm{~B} 2 \mathrm{Ib} / \mathrm{II}$ & 1 & BCEP \\
\hline OV Aps & 151665 & 0.92038 & $1 \mathrm{e}-04$ & 9.7 & 0.2 & 834 & 0.92044 & 8.07 & A7III & 3 & $\mathrm{ACV}$ \\
\hline V846 Ara & 152478 & 0.43861 & $2 \mathrm{e}-05$ & 8.0 & 0.2 & 3649 & $0.60646^{\mathrm{a}}$ & 6.30 & B3Vnpe & 9 & $\mathrm{BE}+\mathrm{GCAS}$ \\
\hline V847 Ara & 152511 & 0.94205 & $1 \mathrm{e}-04$ & 6.5 & 0.1 & 3650 & 0.94213 & 6.53 & B5III & 3 & SPB \\
\hline V884 Sco & 153919 & 3.41141 & $4 \mathrm{e}-04$ & 7.3 & 0.2 & 33700 & 3.41161 & 6.53 & O5F & 10 & $\mathrm{ELL}+\mathrm{HMXB}$ \\
\hline$\ldots$ & 155190 & 1.66574 & $7 \mathrm{e}-04$ & 7.2 & 0.1 & 59550 & $1.66571^{\mathrm{a}}$ & 7.12 & B7III/IV & 3 & SPB \\
\hline V824 Ara & 155555 & 1.68379 & $5 e-05$ & 6.5 & 0.1 & 3627 & 1.68160 & 6.87 & $\mathrm{~K} 1 \mathrm{Vp}$ & 3 & RS \\
\hline
\end{tabular}


Table 7

(Continued)

\begin{tabular}{|c|c|c|c|c|c|c|c|c|c|c|c|}
\hline $\begin{array}{r}\text { Name } \\
\ldots\end{array}$ & $\begin{array}{c}\mathrm{HD} \\
\ldots\end{array}$ & $\begin{array}{c}P \\
\text { (day) }\end{array}$ & $\begin{array}{c}\sigma_{P} \\
\text { (day) }\end{array}$ & $\begin{array}{c}A \\
(\mathrm{mmag})\end{array}$ & $\begin{array}{c}\sigma_{A} \\
(\mathrm{mmag})\end{array}$ & $\begin{array}{c}\text { VSX ID } \\
\ldots\end{array}$ & $\begin{array}{l}P_{\mathrm{VSX}} \\
\text { (day) }\end{array}$ & $\begin{array}{c}V \\
(\mathrm{mag})\end{array}$ & $\begin{array}{c}\text { SpT } \\
\cdots\end{array}$ & $\begin{array}{c}\text { References } \\
\ldots\end{array}$ & $\begin{array}{l}\text { Type } \\
\text { (VSX) }\end{array}$ \\
\hline$\cdots$ & 156853 & 1.15959 & $3 e-04$ & 4.1 & 0.1 & $\cdots$ & 1.15945 & 7.60 & AP SI & 1 & $\mathrm{ACV}$ \\
\hline$\cdots$ & 159041 & 3.80296 & $3 e-03$ & 5.9 & 0.2 & $\cdots$ & $0.09432^{\mathrm{a}}$ & 8.04 & B9Ib/II & 1 & SPB \\
\hline V1092 Sco & 163254 & 0.83175 & $2 \mathrm{e}-04$ & 11.2 & 0.2 & 33908 & 0.83167 & 6.74 & B5Vn & 1 & SPB \\
\hline V692 CrA & 166596 & 1.65010 & $3 e-02$ & 4.1 & 0.2 & 10563 & 1.67000 & 5.46 & B2.5III & 1 & SXARI \\
\hline$\cdots$ & 172416 & 0.82698 & $3 e-04$ & 6.6 & 0.1 & 62979 & 0.99787 & 6.61 & F5V & 1 & GDOR \\
\hline V364 Pav & 175008 & 0.57389 & $5 e-05$ & 6.6 & 0.1 & 25084 & 0.57389 & 6.80 & B9IV/V & 3 & SPB \\
\hline$\ldots$ & 193677 & 0.42930 & $3 e-03$ & 3.2 & 0.1 & 281860 & 0.85857 & 7.60 & $\mathrm{~A} 2 \mathrm{~V}$ & 3 & ROT \\
\hline$\ldots$ & 201247 & 6.32432 & $2 \mathrm{e}-02$ & 2.7 & 0.1 & 63923 & $1.26590^{\mathrm{a}}$ & 6.84 & G0 & 11 & RS \\
\hline$\cdots$ & 209234 & 4.67895 & $1 \mathrm{e}-01$ & 4.6 & 0.1 & $\cdots$ & $0.089454^{\mathrm{a}}$ & 7.87 & G3V & 8 & RS \\
\hline CX Gru & 214291 & 0.87089 & $1 \mathrm{e}-04$ & 11.7 & 0.2 & 14793 & 0.87125 & 6.57 & F7V & 1 & ELL \\
\hline ksi Oct & 215573 & 1.76908 & $3 e-04$ & 5.7 & 0.1 & 20485 & 1.76866 & 5.32 & B6IV & 5 & SPB \\
\hline$\ldots$ & 216668 & 1.78959 & $4 \mathrm{e}-04$ & 6.2 & 0.2 & 305847 & 1.78997 & 7.89 & A1V & 3 & VAR \\
\hline
\end{tabular}

Notes. ACEP: anomalous Cepheid. ACV: $\alpha^{2}$ CVn. BCEP: $\beta$ Cephei. BE: Be star. BY: BY-Draconis type. DPV: double periodic. DSCT: $\delta$ Scuti. ED/ESD: detached EB. ELL: ellipsoidal variable. GCAS: $\gamma$ Cass-type. GDOR: $\gamma$ Dor-type. HMXB: high-mass X-ray binary. LERI: $\lambda$ Eri-type. LPV: long period. M: Mira-type. roAp: chemically peculiar, rapidly oscillating A star. RS: RS CV-type. SDOR: S Doradus-type. SPB: slowly pulsating B-type. SXARI: SX Arietis-type. SXPHE: SXPHEtype variable. TTS: T Tauri Star. WR: Wolf-Rayet.

${ }^{a}$ Rimoldini et al. (2012).

${ }^{\mathrm{b}}$ Reclassified.

References. (1) Houk (1978), (2) Houk (1982), (3) Houk \& Cowley (1975), (4) Cucchiaro et al. (1977), (5) Shara et al. (2009), (6) Keenan \& McNeil (1989), (7) Garrison et al. (1977), (8) Torres et al. (2006), (9) Levenhagen \& Leister (2006), (10) Sota et al. (2014), (11) Gray et al. (2006), (12) Levato et al. (1996).

$H D$ 140566: the final star that showed a different period than the reported VSX period was HD 140566 (previously discussed in Section 4.3). bRing detects a period at 0.08783 days, whereas VSX reports a 193.7000 day period and classified the star as a detached EB (ESD). The bRing light curve does not indicate evidence of an eclipsing system. The period is more indicative of a $\delta$ Scuti, and multiple modes appear in the periodogram. Its spectral type (A5IV) and CMD position (from Figure 4) agree with a $\delta$ Scuti classification. Therefore, this star should be relabeled as a candidate $\delta$ Scuti.

\subsubsection{New Variables}

In Table 8, we list 25 stars that had no known previously published periodicity or classification. A suggested classification was based on the period of the activity, the shape of the light curve, and the spectral type of the star.

The stars HD 103285, HD 73141, and HD 99757 were classified as SPB variables based on their periods and locations in Figure 4. The star HD 76566 (IY Vel) was also observed by Lefèvre et al. (2009), which also detected the 2.10650 day period. However, they left the SPB classification as uncertain; the SPB nature of this star is confirmed with the bRing data. Due to bRing's long baseline, it was possible to detect several long-period variables out to the Nyquist limit of the observations (typically around 266 days). The population of these variables is composed largely of $\mathrm{K}$ giants. For the stars in Table 8, the survey was able to either provide newly measured periods or provide more precise values over VSX periods.

HD 143098: for the G5V star HD 143098, a 8.56567 day period was detected that is indicative of rotation. The star also shows enhanced chromospheric activity $\left(\log R^{\prime} H K=-4.46\right.$, -4.59 Boro Saikia et al. 2018) and strong Li I absorption (EW (Li I $\lambda 6707=60 \mathrm{~mA}$; Torres et al. 2006). Combining this ROT with the $B-V$ color $(0.686 \pm 0.015)$ reported in Hipparcos (Perryman \& ESA 1997), and the age-rotation calibration of Mamajek \& Hillenbrand (2008), we estimate a gyrochronological age of HD 143098 of $0.6 \mathrm{Gyr}$. This is consistent with the other two indicators-both the $\mathrm{Li}$ absorption strength and the chromospheric activity are also very consistent with an age similar to that of the $\sim 0.6-0.8$ Gyr old Hyades (Soderblom 1990; Mamajek \& Hillenbrand 2008).

\subsection{Irregular Variables}

We also detected 17 irregular variables with the bRing photometry, which are listed in Table 9. The table includes previously known irregular variables (Be stars, Mira variables) as well as stars whose bRing light curves showed evidence of variability, but for which no significant period could be converged on.

The majority of these irregular variables exhibited very short bright events (HD 3359, HD 68809, HD 69256, HD 72838, HD 91869, HD 127755, HD 128679, HD 205834). These events were only a few hours in nature and could be degenerate with isolated stochastic events in the bRing data.

The light curve for HD 92063 shows three consecutive bumps in the brightness that extend as bright as $0.3 \mathrm{mag}$. The source of the bumps is likely due to the effects of the nova ASASSN-18fv (Nova Carina 2018, V906 Car) (Stanek et al. 2018), situated only $128^{\prime \prime}$ away from HD 92063. ASASSN$18 \mathrm{fv}$ was alternatively classified as either a classical nova 
Table 8

Other New Variables Detected with bRing

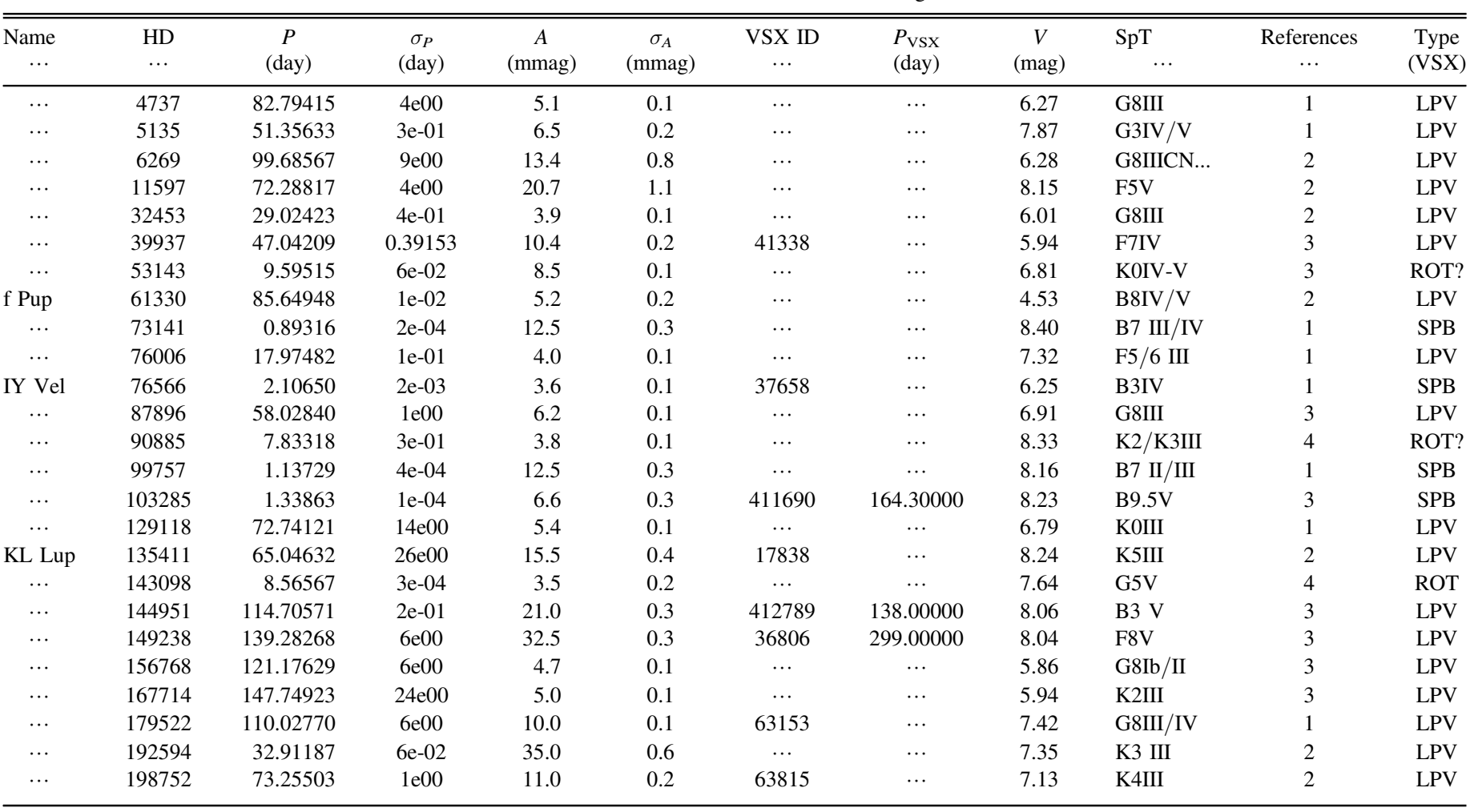

Note. LPV: long period. ROT: rotation period. SPB: slowly pulsating B star.

References. (1) Houk (1978), (2) Houk (1982), (3) Houk \& Cowley (1975), (4) Torres et al. (2006).

Table 9

Irregular Variables Detected with bRing

\begin{tabular}{ccclcc}
\hline \hline $\begin{array}{c}\text { HD } \\
\ldots\end{array}$ & $\begin{array}{c}\text { VSX ID } \\
\cdots\end{array}$ & $\begin{array}{c}P_{\text {VSX }} \\
(\text { day })\end{array}$ & $\begin{array}{c}V \\
(\mathrm{mag})\end{array}$ & $\begin{array}{c}\text { SpT } \\
\cdots\end{array}$ & $\begin{array}{c}\text { References } \\
\cdots\end{array}$ \\
\hline 3359 & $\ldots$ & $\ldots$ & 8.39 & K0V & 1 \\
4229 & $\ldots$ & $\ldots$ & 6.8 & K5III & 2 \\
12440 & $\ldots$ & $\ldots$ & 8.19 & K2III & 2 \\
36597 & $\ldots$ & $\ldots$ & 3.86 & K1II/III & 3 \\
51801 & 55670 & 224.71910 & 7.15 & K2/K3III & 2 \\
68809 & $\ldots$ & $\ldots$ & 7.93 & KOIII & 1 \\
69256 & $\ldots$ & $\ldots$ & 8.17 & K0III & 1 \\
72838 & $\ldots$ & $\ldots$ & 7.25 & K1Ib: & 3 \\
91869 & $\ldots$ & $\ldots$ & 6.9 & G8/K0III+.. & 2 \\
92063 & 43533 & $\ldots$ & 5.08 & K1III & 2 \\
127755 & 58566 & $\ldots$ & 7.66 & K3 III & 2 \\
128679 & $\ldots$ & $\ldots$ & 7.76 & K2 III & 2 \\
139534 & 36907 & 295.39108 & 7.8 & K0II/III & 2 \\
155806 & 33891 & $3.20848^{\text {a }}$ & 5.61 & O8Ve & 4 \\
156468 & 33894 & $\ldots$ & 7.87 & B2V:ne & 3 \\
158864 & 3633 & $\ldots$ & 8.17 & B2 IB/IIeP & 1 \\
205834 & $\ldots$ & $\ldots$ & 8.1 & K0 III & 2 \\
222060 & $\ldots$ & $\ldots$ & 5.99 & K0II/III & 2 \\
\hline
\end{tabular}

Note.

${ }^{a}$ Rimoldini et al. (2012).

References. (1) Houk (1978), (2) Houk \& Cowley (1975), (3) Houk (1982), (4) Sota et al. (2014).

(Luckas 2018; Rabus \& Prieto 2018) or a young stellar object that underwent a burst of accretion (Strader et al. 2018). Given the proximity of the nova compared to the bRing pixel size, the light curve of HD 92063 is likely corrupted by ASASSN-18fv.
bRing also observed a few isolated dimming events in the stars HD 4229, HD 12440, HD 35324, and HD 222060; however, the events could not be verified as real, significant events due to their short duration and noisy characteristics. We also identified HD 36597, HD 51801, HD 139534, and HD 159468 as semi-regular variables in the bRing data.

HD 155806 (V1075 Sco) and HD 158864 (V830 Ara): V1075 Sco (O8V star classified as a Be in VSX) shows significant variability (amplitude of $0.05 \mathrm{mag}$ ) over the first 100 days of bRing data. After disappearing from the bRing data for about 100 more days, the star reappears as roughly constant. This is likely just a symptom of the irregularity exhibited by these stars. Since HD 155806 is not listed as a GCAS in any source, bRing simply classified this as an irregular star. V830 Ara showed several significant brightening events $(\simeq 0.2 \mathrm{mag})$ throughout the 500 days of observation. A representative period could not be converged on, so this was classified as irregular.

In Mamajek et al. (2012), the authors detected an unusually deep eclipsing event that took place over $\sim 50$ days in the J1407 system; this light curve has been subsequently modeled as a circumplanetary ring system (van Werkhoven et al. 2014; Kenworthy \& Mamajek 2015). Follow-up observations and a study of the archival photometry have attempted to deduce the periodic nature of this event, but an additional event has yet to be confirmed (Mentel et al. 2018). While searching for variables in the bRing data, evidence of such eclipses in other stars were also searched for. Unfortunately, no other eclipses of similar length or depth were detected. 


\section{Conclusion}

The bRing survey of $\beta$ Pictoris included nearly continuous photometric monitoring of $10,000+$ bright variables in the southern sky (Stuik et al. 2017; Mellon et al. 2019b, 2019a). This paper reports on the variability of the bright ( $V \simeq 4-8 \mathrm{mag}$ ) stars observed during the bRing survey, and provides improved periods (and sometimes classifications) for many known variables, identifies new variables missed by previous surveys, and provides classifications for some VSX candidate variables. The light curves were also examined for any evidence of transits by circumstellar or circumplanetary dust disks or ring systems analogous to J1407 (V1400 Cen), but no such cases were observed.

Of the 16,762 analyzed in this survey, 353 stars were detected as variable $(80 \%$ were previously known and $20 \%$ were new detections or classifications). These stars were separated by variability into several tables where identifying information and the bRing periods and amplitudes were provided. We provided a brief discussion on the stars whose periods or classifications deviated from those established by the VSX catalog or other surveys. We also provided brief discussion on the newly detected variables and the stars that should be reclassified to better fit their period, light-curve shape, and spectral type. bRing was able to measure the O'Connell effect in 18 of the contact binaries in this survey; only 2 of these had previously shown the asymmetry in their light curves. This survey also detected 18 irregular variables, which were briefly discussed. The results from this survey of the bRing time-series photometry provides initial assessments of the variability parameters for bright southern stars, and may provide opportunities for further study to constrain the nature of these new and reclassified variable stars.

S.N.M. is a U.S. Department of Defense SMART scholar sponsored by the U.S. Navy through NIWC-Atlantic. The results reported herein benefitted from collaborations and/or information exchange within NASA's Nexus for Exoplanet System Science (NExSS) research coordination network sponsored by NASA's Science Mission Directorate. Part of this research was carried out at the Jet Propulsion Laboratory, California Institute of Technology, under a contract with NASA. The authors would like to acknowledge the support staff at both the South African Astronomical Observatory and Siding Spring Observatory for keeping both bRing stations maintained and running. Construction of the bRing observatory to be sited at Siding Springs, Australia would not be possible without a University of Rochester University Research Award, help from Mike Culver and Rich Sarkis (UR), and generous donations of time, services, and materials from Joe and Debbie Bonvissuto of Freight Expediters, Michael Akkaoui and his team at Tanury Industries, Robert Harris and Michael Fay at BCI, Koch Division, Mark Paup, Dave Mellon, and Ray Miller and the Zippo Tool Room. This research has made use of the International Variable Star Index (VSX) database, operated at AAVSO, Cambridge, Massachusetts, USA. This research has made use of the VizieR catalog access tool, CDS, Strasbourg, France (doi:10.26093/cds/vizier). This research has made use of the SIMBAD database, operated at CDS, Strasbourg, France. We acknowledge with thanks the variable star observations from the AAVSO International Database contributed by observers worldwide and used in this research.

Facilities: bRing-SA, bRing-AU, AAVSO.

Software: Python 3.7.3 (Rossum 1995), scipy (Jones et al. 2001), matplotlib (Hunter 2007), numpy (Stéfan van der Walt \& Varoquaux 2011), astropy (The Astropy Collaboration et al. 2018).

\section{Appendix}

Table 10 in the Appendix contains the adopted and calculated stellar parameters used to generate the color-magnitude diagram in Figure 4.

Table 10

Adopted and Calculated Stellar Parameters

\begin{tabular}{|c|c|c|c|c|c|c|c|c|c|c|}
\hline $\begin{array}{r}\text { Name } \\
\ldots\end{array}$ & $\begin{array}{c}l \\
(\mathrm{deg})\end{array}$ & $\begin{array}{c}b \\
\text { (deg) }\end{array}$ & $\begin{array}{c}\varpi \\
\text { (mas) }\end{array}$ & $\begin{array}{c}B \\
(\mathrm{mag})\end{array}$ & $\begin{array}{c}V \\
(\mathrm{mag})\end{array}$ & $\begin{array}{c}(B-V) \\
(\mathrm{mag})\end{array}$ & $\begin{array}{c}E(B-V) \\
(\mathrm{mag})\end{array}$ & $\begin{array}{c}(B-V)_{0} \\
(\mathrm{mag})\end{array}$ & $\begin{array}{c}A_{V} \\
(\mathrm{mag})\end{array}$ & $\begin{array}{c}M_{V} \\
(\mathrm{mag})\end{array}$ \\
\hline HD 3112 & 305.008486 & -45.789165 & 7.162 & 6.359 & 6.109 & 0.250 & 0.005 & 0.245 & 0.015 & 0.369 \\
\hline HD 3359 & 309.594473 & -67.800879 & 23.039 & 9.150 & 8.370 & 0.780 & 0.001 & 0.779 & 0.003 & 5.179 \\
\hline HD 3463 & 304.865029 & -47.640183 & 5.377 & 8.240 & 8.040 & 0.200 & 0.008 & 0.192 & 0.025 & 1.668 \\
\hline HD 4229 & 303.190503 & -31.421460 & 7.005 & 8.099 & 6.808 & 1.291 & 0.008 & 1.283 & 0.026 & 1.009 \\
\hline HD 4737 & 304.207519 & -70.424430 & 7.996 & 7.175 & 6.277 & 0.898 & 0.002 & 0.896 & 0.006 & 0.785 \\
\hline HD 5135 & 302.466765 & -65.577096 & 5.346 & 8.570 & 7.880 & 0.690 & 0.004 & 0.686 & 0.013 & 1.508 \\
\hline HD 6269 & 256.206003 & -86.456746 & 7.841 & 7.213 & 6.283 & 0.930 & 0.002 & 0.928 & 0.006 & 0.749 \\
\hline HD 6882 & 297.833132 & -61.714404 & 10.920 & 3.908 & 4.014 & -0.106 & 0.002 & -0.108 & 0.006 & -0.801 \\
\hline HD 74422 & 278.644521 & -12.886437 & 3.120 & 8.390 & 8.120 & 0.270 & 0.087 & 0.183 & 0.268 & 0.323 \\
\hline HD 8351 & 271.660105 & -78.144661 & 7.234 & 6.960 & 6.698 & 0.262 & 0.002 & 0.260 & 0.006 & 0.989 \\
\hline HD 11413 & 280.699247 & -64.277086 & 12.726 & 6.080 & 5.929 & 0.151 & 0.001 & 0.150 & 0.003 & 1.449 \\
\hline HD 11597 & 239.679217 & -75.489274 & 12.648 & 8.610 & 8.150 & 0.460 & 0.001 & 0.459 & 0.003 & 3.657 \\
\hline HD 12284 & 246.808665 & -73.257393 & 4.900 & 9.448 & 9.108 & 0.340 & 0.004 & 0.336 & 0.012 & 2.547 \\
\hline HD 12440 & 296.850833 & -42.225420 & 2.537 & 9.500 & 8.200 & 1.300 & 0.030 & 1.270 & 0.098 & 0.124 \\
\hline HD 13397 & 272.939621 & -63.724270 & 3.755 & 8.770 & 7.740 & 1.030 & 0.008 & 1.022 & 0.026 & 0.588 \\
\hline HD 16589 & 245.541212 & -65.100855 & 17.948 & 6.995 & 6.483 & 0.512 & 0.001 & 0.511 & 0.003 & 2.750 \\
\hline HD 17084 & 244.720178 & -64.175500 & 24.310 & 8.770 & 8.055 & 0.715 & 0.000 & 0.715 & 0.000 & 4.984 \\
\hline HD 17653 & 290.780175 & -43.096853 & 17.029 & 7.120 & 6.660 & 0.460 & 0.002 & 0.458 & 0.006 & 2.810 \\
\hline HD 17755 & 283.036786 & -49.418163 & 10.366 & 8.480 & 8.060 & 0.420 & 0.002 & 0.418 & 0.006 & 3.132 \\
\hline HD 20232 & 243.589022 & -58.176492 & 12.584 & 7.040 & 6.880 & 0.160 & 0.001 & 0.159 & 0.003 & 2.376 \\
\hline HIP 21213 & 241.248424 & -42.783693 & 7.500 & 7.800 & 7.630 & 0.170 & 0.001 & 0.169 & 0.003 & 2.002 \\
\hline HD 21765 & 276.560889 & -47.102603 & 9.443 & 8.351 & 7.890 & 0.461 & 0.002 & 0.459 & 0.006 & 2.759 \\
\hline HD 25860 & 255.319180 & -47.497443 & 7.153 & 6.826 & 6.615 & 0.211 & 0.002 & 0.209 & 0.006 & 0.881 \\
\hline HD 28837 & 245.182260 & -43.294769 & 6.527 & 7.400 & 7.010 & 0.390 & 0.002 & 0.388 & 0.006 & 1.077 \\
\hline
\end{tabular}


Table 10

(Continued)

\begin{tabular}{|c|c|c|c|c|c|c|c|c|c|c|}
\hline $\begin{array}{r}\text { Name } \\
\ldots\end{array}$ & $\begin{array}{c}l \\
(\mathrm{deg})\end{array}$ & $\begin{array}{c}b \\
(\mathrm{deg})\end{array}$ & $\begin{array}{c}\varpi \\
\text { (mas) }\end{array}$ & $\begin{array}{c}B \\
(\mathrm{mag})\end{array}$ & $\begin{array}{c}V \\
(\mathrm{mag})\end{array}$ & $\begin{array}{c}(B-V) \\
(\mathrm{mag})\end{array}$ & $\begin{array}{c}E(B-V) \\
\quad(\mathrm{mag})\end{array}$ & $\begin{array}{l}(B-V)_{0} \\
\quad(\mathrm{mag})\end{array}$ & $\begin{array}{c}A_{V} \\
\text { (mag) }\end{array}$ & $\begin{array}{c}M_{V} \\
\text { (mag) }\end{array}$ \\
\hline HD 30551 & 255.898732 & -40.347787 & 0.870 & 8.990 & 6.350 & 2.640 & 0.012 & 2.628 & 0.042 & -3.995 \\
\hline HD 30861 & 253.109163 & -39.985308 & 4.691 & 7.580 & 7.440 & 0.140 & 0.003 & 0.137 & 0.009 & 0.787 \\
\hline HD 31407 & 264.090685 & -38.718807 & 0.967 & 7.470 & 7.690 & -0.220 & 0.013 & -0.233 & 0.039 & -2.422 \\
\hline HD 32453 & 243.737596 & -37.381308 & 8.017 & 6.918 & 6.007 & 0.911 & 0.001 & 0.910 & 0.003 & 0.524 \\
\hline HD 32846 & 238.873189 & -36.257079 & 10.170 & 6.607 & 6.314 & 0.293 & 0.001 & 0.292 & 0.003 & 1.348 \\
\hline HD 33331 & 250.226912 & -36.738948 & 3.390 & 6.806 & 6.892 & -0.086 & 0.005 & -0.091 & 0.015 & -0.472 \\
\hline HD 34349 & 275.285824 & -34.809895 & 16.128 & 7.450 & 7.050 & 0.400 & 0.001 & 0.399 & 0.003 & 3.085 \\
\hline HD 34802 & 289.295356 & -31.915956 & 5.409 & 8.850 & 7.770 & 1.080 & 0.030 & 1.050 & 0.097 & 1.339 \\
\hline HD 35324 & 282.662535 & -33.019396 & 4.625 & 9.060 & 7.720 & 1.340 & 0.023 & 1.317 & 0.075 & 0.971 \\
\hline HIP 35815 & 251.367772 & -11.077202 & 4.515 & 8.200 & 7.840 & 0.360 & 0.008 & 0.352 & 0.025 & 1.088 \\
\hline HD 36597 & 239.889723 & -30.875387 & 12.461 & 5.010 & 3.870 & 1.140 & 0.001 & 1.139 & 0.003 & -0.655 \\
\hline HD 36705 & 275.300830 & -33.045550 & 65.320 & 7.856 & 6.999 & 0.857 & 0.000 & 0.857 & 0.000 & 6.074 \\
\hline HD 37350 & 271.733779 & -32.774457 & 3.112 & 4.580 & 3.760 & 0.820 & 0.014 & 0.806 & 0.045 & -3.819 \\
\hline HD 37513 & 287.845391 & -31.095793 & 9.644 & 8.800 & 8.240 & 0.560 & 0.003 & 0.557 & 0.009 & 3.152 \\
\hline HD 37909 & 293.876606 & -29.872461 & 6.892 & 8.510 & 8.260 & 0.250 & 0.008 & 0.242 & 0.025 & 2.427 \\
\hline HD 39244 & 252.963719 & -29.692239 & 6.456 & 7.770 & 7.530 & 0.240 & 0.002 & 0.238 & 0.006 & 1.574 \\
\hline HD 39764 & 239.361225 & -26.094313 & 9.666 & 4.720 & 4.870 & -0.150 & 0.001 & -0.151 & 0.003 & -0.207 \\
\hline HD 39917 & 250.029969 & -28.401647 & 5.307 & 8.660 & 7.906 & 0.754 & 0.003 & 0.751 & 0.010 & 1.521 \\
\hline HD 39937 & 265.481354 & -30.410684 & 8.536 & 6.582 & 5.948 & 0.634 & 0.001 & 0.633 & 0.003 & 0.601 \\
\hline HD 41846 & 280.357024 & -29.637540 & 5.192 & 8.470 & 8.110 & 0.360 & 0.014 & 0.346 & 0.044 & 1.643 \\
\hline HD 42504 & 262.654416 & -27.941484 & 2.656 & 8.690 & 7.700 & 0.990 & 0.022 & 0.968 & 0.071 & -0.250 \\
\hline HD 42933 & 263.302876 & -27.683668 & 2.510 & 4.580 & 4.810 & -0.230 & 0.027 & -0.257 & 0.081 & -3.273 \\
\hline HD 43518 & 268.921715 & -27.999235 & 3.870 & 8.700 & 7.410 & 1.290 & 0.009 & 1.281 & 0.029 & 0.319 \\
\hline HD 43898 & 243.230633 & -22.045071 & 6.925 & 8.160 & 7.870 & 0.290 & 0.002 & 0.288 & 0.006 & 2.066 \\
\hline HD 46291 & 282.691975 & -27.681412 & 3.468 & 9.370 & 8.261 & 1.109 & 0.040 & 1.069 & 0.129 & 0.832 \\
\hline HD 46586 & 256.476067 & -22.936854 & 8.882 & 8.320 & 8.030 & 0.290 & 0.001 & 0.289 & 0.003 & 2.770 \\
\hline HD 46697 & 268.279215 & -25.562813 & 6.924 & 8.790 & 7.739 & 1.051 & 0.003 & 1.048 & 0.010 & 1.931 \\
\hline HD 46978 & 295.054679 & -27.916876 & 5.090 & 8.480 & 8.160 & 0.320 & 0.073 & 0.247 & 0.226 & 1.468 \\
\hline HD 49877 & 265.125175 & -22.645861 & 4.812 & 7.190 & 5.610 & 1.580 & 0.007 & 1.573 & 0.023 & -1.001 \\
\hline HD 51801 & 281.264333 & -25.361915 & 2.414 & 8.510 & 7.140 & 1.370 & 0.049 & 1.321 & 0.160 & -1.107 \\
\hline HD 52993 & 246.244795 & -13.439255 & 4.336 & 6.416 & 6.569 & -0.153 & 0.007 & -0.160 & 0.021 & -0.267 \\
\hline HD 53143 & 271.654979 & -22.592123 & 54.466 & 7.609 & 6.803 & 0.806 & 0.000 & 0.806 & 0.000 & 5.484 \\
\hline HD 54579 & 246.104700 & -12.009698 & 15.469 & 8.940 & 8.029 & 0.911 & 0.001 & 0.910 & 0.003 & 3.973 \\
\hline HD 55173 & 242.507518 & -9.724266 & 1.014 & 7.320 & 7.490 & -0.170 & 0.049 & -0.219 & 0.148 & -2.628 \\
\hline HD 56142 & 248.137346 & -11.516440 & 4.202 & 8.140 & 7.590 & 0.550 & 0.008 & 0.542 & 0.025 & 0.682 \\
\hline HD 56146 & 270.588989 & -20.723588 & 2.261 & 8.050 & 8.090 & -0.040 & 0.091 & -0.131 & 0.276 & -0.414 \\
\hline HD 56910 & 269.431682 & -19.896621 & 6.452 & 7.100 & 6.840 & 0.260 & 0.005 & 0.255 & 0.015 & 0.873 \\
\hline HD 57969 & 267.448737 & -18.489199 & 13.850 & 6.670 & 6.560 & 0.110 & 0.001 & 0.109 & 0.003 & 2.264 \\
\hline HD 58635 & 249.869298 & -9.996722 & 5.520 & 7.100 & 6.810 & 0.290 & 0.006 & 0.284 & 0.019 & 0.501 \\
\hline HD 60168 & 249.247507 & -8.119752 & 4.071 & 6.540 & 6.617 & -0.077 & 0.010 & -0.087 & 0.030 & -0.365 \\
\hline HD 60559 & 253.130100 & -9.785495 & 4.435 & 6.130 & 6.247 & -0.117 & 0.011 & -0.128 & 0.033 & -0.552 \\
\hline HD 60649 & 265.536902 & -15.781967 & 1.868 & 6.900 & 7.000 & -0.100 & 0.109 & -0.209 & 0.329 & -1.972 \\
\hline HD 61330 & 248.978598 & -6.669998 & 9.050 & 4.440 & 4.530 & -0.090 & 0.002 & -0.092 & 0.006 & -0.693 \\
\hline HD 61644 & 273.717460 & -18.682117 & 2.270 & 8.480 & 8.410 & 0.070 & 0.138 & -0.068 & 0.419 & -0.229 \\
\hline HD 63203 & 269.250591 & -15.590387 & 1.914 & 8.300 & 8.320 & -0.020 & 0.111 & -0.131 & 0.336 & -0.606 \\
\hline HD 63786 & 250.416015 & -4.687935 & 6.539 & 5.886 & 5.936 & -0.050 & 0.005 & -0.055 & 0.015 & -0.002 \\
\hline HD 64503 & 253.899409 & -5.925270 & 5.030 & 4.301 & 4.474 & -0.173 & 0.010 & -0.183 & 0.030 & -2.048 \\
\hline HD 64722 & 267.614913 & -13.538668 & 2.313 & 5.529 & 5.680 & -0.151 & 0.140 & -0.291 & 0.420 & -2.919 \\
\hline HD 65592 & 255.495365 & -5.718947 & 0.802 & 8.130 & 7.370 & 0.760 & 0.356 & 0.404 & 1.110 & -4.219 \\
\hline HD 65818 & 263.475590 & -10.279223 & 3.400 & 4.240 & 4.410 & -0.170 & 0.054 & -0.224 & 0.163 & -3.095 \\
\hline HD 66235 & 260.267567 & -7.955540 & 2.508 & 7.540 & 7.670 & -0.130 & 0.029 & -0.159 & 0.088 & -0.421 \\
\hline HD 66260 & 274.638128 & -16.135648 & 5.388 & 7.760 & 7.460 & 0.300 & 0.016 & 0.284 & 0.050 & 1.068 \\
\hline HD 66503 & 258.189795 & -6.404198 & 2.190 & 8.126 & 8.220 & -0.094 & 0.025 & -0.119 & 0.076 & -0.154 \\
\hline HD 66623 & 251.745048 & -2.180114 & 12.137 & 8.680 & 8.143 & 0.537 & 0.001 & 0.536 & 0.003 & 3.560 \\
\hline HD 66768 & 269.364185 & -12.874937 & 2.557 & 6.660 & 6.690 & -0.030 & 0.187 & -0.217 & 0.564 & -1.835 \\
\hline HD 68556 & 263.228698 & -7.694949 & 0.616 & 8.780 & 8.160 & 0.620 & 0.120 & 0.500 & 0.376 & -3.268 \\
\hline HD 68808 & 262.440856 & -6.959550 & 1.217 & 6.350 & 5.760 & 0.590 & 0.066 & 0.524 & 0.207 & -4.021 \\
\hline HD 68809 & 263.924453 & -7.933014 & 2.491 & 9.070 & 7.930 & 1.140 & 0.042 & 1.098 & 0.136 & -0.224 \\
\hline HD 68860 & 252.427475 & -0.187155 & 0.584 & 8.000 & 6.700 & 1.300 & 0.202 & 1.098 & 0.653 & -5.120 \\
\hline HD 69213 & 260.894205 & -5.528365 & 9.858 & 6.980 & 6.700 & 0.280 & 0.002 & 0.278 & 0.006 & 1.663 \\
\hline HD 69256 & 262.640578 & -6.653319 & 3.475 & 9.310 & 8.200 & 1.110 & 0.023 & 1.087 & 0.074 & 0.830 \\
\hline HD 69342 & 258.843122 & -4.020185 & $\ldots$ & 8.240 & 8.060 & 0.180 & $\ldots$ & $\ldots$ & $\ldots$ & $\ldots$ \\
\hline HD 69879 & 249.199313 & 3.217249 & 7.055 & 7.475 & 6.422 & 1.053 & 0.004 & 1.049 & 0.013 & 0.652 \\
\hline
\end{tabular}


Table 10

(Continued)

\begin{tabular}{|c|c|c|c|c|c|c|c|c|c|c|}
\hline $\begin{array}{r}\text { Name } \\
\ldots\end{array}$ & $\begin{array}{c}l \\
(\mathrm{deg})\end{array}$ & $\begin{array}{c}b \\
\text { (deg) }\end{array}$ & $\begin{array}{c}\varpi \\
\text { (mas) }\end{array}$ & $\begin{array}{c}B \\
(\mathrm{mag})\end{array}$ & $\begin{array}{c}V \\
(\mathrm{mag})\end{array}$ & $\begin{array}{c}(B-V) \\
(\mathrm{mag})\end{array}$ & $\begin{array}{c}E(B-V) \\
\quad(\mathrm{mag})\end{array}$ & $\begin{array}{c}(B-V)_{0} \\
(\mathrm{mag})\end{array}$ & $\begin{array}{c}A_{V} \\
\text { (mag) }\end{array}$ & $\begin{array}{c}M_{V} \\
\text { (mag) }\end{array}$ \\
\hline HD 69882 & 259.498710 & -3.908606 & 0.525 & 7.480 & 7.170 & 0.310 & 0.280 & 0.030 & 0.855 & -5.085 \\
\hline HD 70999 & 256.821157 & -0.612875 & 1.472 & 7.930 & 8.050 & -0.120 & 0.113 & -0.233 & 0.340 & -1.450 \\
\hline HD 71302 & 260.502870 & -2.888402 & 1.820 & 5.900 & 6.020 & -0.120 & 0.065 & -0.185 & 0.196 & -2.876 \\
\hline HD 71487 & 257.618591 & -0.538006 & 5.820 & 6.660 & 6.500 & 0.160 & 0.009 & 0.151 & 0.028 & 0.297 \\
\hline HD 71801 & 254.604570 & 2.030543 & 1.626 & 5.600 & 5.740 & -0.140 & 0.062 & -0.202 & 0.187 & -3.391 \\
\hline HD 72275 & 275.249545 & -12.285240 & 0.760 & 8.140 & 7.310 & 0.830 & 0.150 & 0.680 & 0.474 & -3.760 \\
\hline HD 72698 & 274.678126 & -11.515123 & 2.634 & 8.130 & 8.060 & 0.070 & 0.103 & -0.033 & 0.314 & -0.151 \\
\hline HD 72754 & 266.826694 & -5.815158 & 0.582 & 9.090 & 8.880 & 0.210 & 0.231 & -0.021 & 0.704 & -2.999 \\
\hline HD 72838 & 266.517046 & -5.480196 & 0.613 & 9.020 & 7.270 & 1.750 & 0.216 & 1.534 & 0.714 & -4.505 \\
\hline HD 72878 & 271.659765 & -9.204825 & 1.900 & 7.520 & 7.460 & 0.060 & 0.110 & -0.050 & 0.335 & -1.481 \\
\hline HD 72979 & 284.687144 & -17.945936 & 4.154 & 7.900 & 7.700 & 0.200 & 0.045 & 0.155 & 0.138 & 0.654 \\
\hline HD 73141 & 267.079253 & -5.553821 & 1.925 & 8.310 & 8.410 & -0.100 & 0.098 & -0.198 & 0.296 & -0.464 \\
\hline HD 73340 & 268.266544 & -6.176067 & 6.705 & 5.651 & 5.782 & -0.131 & 0.005 & -0.136 & 0.015 & -0.101 \\
\hline HD 73502 & 262.882000 & -1.910762 & 0.547 & 8.507 & 7.260 & 1.247 & 0.186 & 1.061 & 0.600 & -4.651 \\
\hline HD 73699 & 259.795991 & 0.695547 & 1.004 & 7.620 & 7.600 & 0.020 & 0.087 & -0.067 & 0.264 & -2.656 \\
\hline HD 73882 & 260.181611 & 0.643141 & 2.170 & 7.590 & 7.190 & 0.400 & 0.036 & 0.364 & 0.112 & -1.240 \\
\hline HD 74195 & 270.250715 & -6.800198 & 6.610 & 3.440 & 3.630 & -0.190 & 0.005 & -0.195 & 0.015 & -2.284 \\
\hline HD 74531 & 266.684501 & -3.612202 & 1.331 & 7.080 & 7.230 & -0.150 & 0.100 & -0.250 & 0.301 & -2.450 \\
\hline HD 74712 & 266.194768 & -3.002906 & 0.258 & 9.580 & 8.320 & 1.260 & 0.230 & 1.030 & 0.741 & -5.361 \\
\hline HD 74884 & 265.493657 & -2.179077 & 0.409 & 9.190 & 8.330 & 0.860 & 0.312 & 0.548 & 0.980 & -4.593 \\
\hline HD 75747 & 292.550759 & -21.631893 & 10.096 & 6.280 & 6.070 & 0.210 & 0.005 & 0.205 & 0.015 & 1.075 \\
\hline HD 76006 & 268.697008 & -3.333926 & 0.677 & 8.880 & 8.550 & 0.330 & 0.155 & 0.175 & 0.477 & -2.773 \\
\hline HD 76566 & 265.641514 & 0.054159 & 2.601 & 6.100 & 6.260 & -0.160 & 0.025 & -0.185 & 0.075 & -1.739 \\
\hline HD 76640 & 275.750650 & -8.474583 & 4.580 & 6.257 & 6.353 & -0.096 & 0.029 & -0.125 & 0.088 & -0.431 \\
\hline HD 76875 & 269.870923 & -3.179071 & 2.681 & 8.830 & 7.720 & 1.110 & 0.031 & 1.079 & 0.100 & -0.238 \\
\hline HD 77347 & 273.102827 & -5.381860 & 4.675 & 7.840 & 7.580 & 0.260 & 0.019 & 0.241 & 0.059 & 0.870 \\
\hline HD 77464 & 271.159400 & -3.523334 & 1.534 & 6.550 & 6.690 & -0.140 & 0.054 & -0.194 & 0.163 & -2.544 \\
\hline HD 77581 & 263.058292 & 3.929854 & 0.384 & 7.370 & 6.870 & 0.500 & 0.142 & 0.358 & 0.442 & -5.651 \\
\hline HD 77653 & 271.749887 & -3.811274 & 8.850 & 5.171 & 5.295 & -0.124 & 0.003 & -0.127 & 0.009 & 0.021 \\
\hline HD 77669 & 265.662442 & 0.121400 & 1.955 & 8.050 & 8.100 & -0.050 & 0.040 & -0.090 & 0.121 & -0.565 \\
\hline HD 78165 & 264.674744 & 3.317533 & 2.912 & 7.820 & 7.610 & 0.210 & 0.021 & 0.189 & 0.065 & -0.134 \\
\hline HD 78405 & 272.634839 & -3.739767 & 1.764 & 8.150 & 8.260 & -0.110 & 0.049 & -0.159 & 0.148 & -0.655 \\
\hline HD 78763 & 285.437818 & -14.797121 & 1.902 & 8.270 & 8.310 & -0.040 & 0.066 & -0.106 & 0.200 & -0.494 \\
\hline HD 78801 & 271.863164 & -2.558817 & 1.007 & 8.820 & 7.690 & 1.130 & 0.076 & 1.054 & 0.245 & -2.539 \\
\hline HD 79039 & 269.132131 & 0.321652 & 2.273 & 6.692 & 6.812 & -0.120 & 0.029 & -0.149 & 0.088 & -1.493 \\
\hline HD 81222 & 276.569793 & -4.194508 & 0.911 & 8.290 & 7.570 & 0.720 & 0.217 & 0.503 & 0.680 & -3.312 \\
\hline HD 81654 & 278.728196 & -5.905462 & 0.916 & 7.880 & 7.880 & 0.000 & 0.176 & -0.176 & 0.532 & -2.843 \\
\hline HD 81771 & 288.159479 & -15.200061 & 3.475 & 7.970 & 7.760 & 0.210 & 0.086 & 0.124 & 0.264 & 0.201 \\
\hline HD 82484 & 266.475505 & 8.320205 & 2.664 & 8.300 & 8.090 & 0.210 & 0.039 & 0.171 & 0.120 & 0.098 \\
\hline HD 82829 & 270.382574 & 4.770436 & 5.973 & 8.050 & 7.810 & 0.240 & 0.012 & 0.228 & 0.037 & 1.654 \\
\hline HD 84400 & 275.668170 & 1.411384 & 2.413 & 6.061 & 6.168 & -0.107 & 0.043 & -0.150 & 0.130 & -2.050 \\
\hline HD 84416 & 285.886712 & -10.535299 & 6.407 & 6.390 & 6.320 & 0.070 & 0.014 & 0.056 & 0.043 & 0.310 \\
\hline HD 84810 & 283.199003 & -7.003808 & 0.777 & 4.330 & 3.400 & 0.930 & 0.156 & 0.774 & 0.496 & -7.643 \\
\hline HD 85037 & 275.356375 & 2.830386 & 7.345 & 6.660 & 6.530 & 0.130 & 0.008 & 0.122 & 0.025 & 0.835 \\
\hline HD 85185 & 274.208898 & 4.519312 & 2.817 & 8.040 & 8.010 & 0.030 & 0.032 & -0.002 & 0.098 & 0.161 \\
\hline HD 85871 & 279.410387 & -0.870275 & 1.185 & 8.900 & 6.491 & 2.409 & 0.091 & 2.318 & 0.313 & -3.453 \\
\hline HD 85953 & 276.869760 & 2.510135 & 1.684 & 5.777 & 5.932 & -0.155 & 0.056 & -0.211 & 0.169 & -3.105 \\
\hline HD 86118 & 281.478387 & -3.120892 & 1.341 & 6.470 & 6.640 & -0.170 & 0.100 & -0.270 & 0.301 & -3.024 \\
\hline HD 86441 & 281.231910 & -2.339378 & 1.033 & 7.490 & 7.520 & -0.030 & 0.163 & -0.193 & 0.492 & -2.901 \\
\hline HD 87072 & 286.854973 & -8.911832 & 0.858 & 9.070 & 8.290 & 0.780 & 0.155 & 0.625 & 0.489 & -2.532 \\
\hline HD 87896 & 285.865065 & -6.593891 & 6.670 & 7.820 & 6.910 & 0.910 & 0.015 & 0.895 & 0.048 & 0.983 \\
\hline HD 88278 & 295.294369 & -18.743258 & 4.663 & 7.550 & 7.310 & 0.240 & 0.175 & 0.065 & 0.536 & 0.118 \\
\hline HD 88824 & 279.384303 & 4.265916 & 20.186 & 5.519 & 5.265 & 0.254 & 0.003 & 0.251 & 0.009 & 1.781 \\
\hline HD 88825 & 284.272213 & -2.915781 & 1.455 & 5.997 & 6.087 & -0.090 & 0.077 & -0.167 & 0.233 & -3.331 \\
\hline HD 89611 & 277.070166 & 9.457440 & 2.421 & 7.980 & 7.960 & 0.020 & 0.021 & -0.001 & 0.064 & -0.184 \\
\hline HD 89841 & 282.568898 & 1.474929 & 0.610 & 9.710 & 7.860 & 1.850 & 0.177 & 1.673 & 0.589 & -3.803 \\
\hline HD 90000 & 279.472330 & 6.656732 & 1.299 & 7.420 & 7.560 & -0.140 & 0.070 & -0.210 & 0.211 & -2.084 \\
\hline HD 90611 & 279.233514 & 8.442773 & 9.683 & 6.830 & 6.550 & 0.280 & 0.004 & 0.276 & 0.012 & 1.468 \\
\hline HD 90885 & 282.046388 & 4.407698 & 29.660 & 9.140 & 8.331 & 0.809 & 0.001 & 0.808 & 0.003 & 5.689 \\
\hline HD 90941 & 280.261842 & 7.510635 & 1.770 & 8.400 & 7.810 & 0.590 & 0.061 & 0.529 & 0.191 & -1.142 \\
\hline HD 91519 & 273.992190 & 18.937293 & 4.197 & 7.980 & 7.700 & 0.280 & 0.030 & 0.250 & 0.093 & 0.722 \\
\hline HD 91869 & 285.492104 & 0.502151 & 2.070 & 7.790 & 6.910 & 0.880 & 0.046 & 0.834 & 0.147 & -1.657 \\
\hline HD 92063 & 286.572675 & -1.053904 & 13.237 & 6.249 & 5.088 & 1.161 & 0.010 & 1.151 & 0.032 & 0.664 \\
\hline
\end{tabular}


Table 10

(Continued)

\begin{tabular}{|c|c|c|c|c|c|c|c|c|c|c|}
\hline $\begin{array}{r}\text { Name } \\
\ldots\end{array}$ & $\begin{array}{c}l \\
(\mathrm{deg})\end{array}$ & $\begin{array}{c}b \\
(\mathrm{deg})\end{array}$ & $\begin{array}{c}\varpi \\
\text { (mas) }\end{array}$ & $\begin{array}{c}B \\
(\mathrm{mag})\end{array}$ & $\begin{array}{c}V \\
(\mathrm{mag})\end{array}$ & $\begin{array}{c}(B-V) \\
(\mathrm{mag})\end{array}$ & $\begin{array}{c}E(B-V) \\
\quad(\mathrm{mag})\end{array}$ & $\begin{array}{l}(B-V)_{0} \\
(\mathrm{mag})\end{array}$ & $\begin{array}{c}A_{V} \\
\text { (mag) }\end{array}$ & $\begin{array}{c}M_{V} \\
(\mathrm{mag})\end{array}$ \\
\hline HD 92287 & 285.630996 & 1.065114 & 2.666 & 5.749 & 5.876 & -0.127 & 0.038 & -0.165 & 0.115 & -2.109 \\
\hline HD 92762 & 296.898420 & -18.231703 & 7.730 & 8.060 & 7.810 & 0.250 & 0.009 & 0.241 & 0.028 & 2.223 \\
\hline HD 93130 & 287.568572 & -0.859319 & 0.359 & 8.310 & 8.040 & 0.270 & 0.397 & -0.127 & 1.202 & -5.389 \\
\hline HD 93203 & 286.547631 & 1.212855 & 0.512 & 7.700 & 6.870 & 0.830 & 0.230 & 0.600 & 0.724 & -5.308 \\
\hline HD 93486 & 298.409551 & -20.324017 & 5.724 & 8.540 & 8.090 & 0.450 & 0.034 & 0.416 & 0.106 & 1.773 \\
\hline HD 93668 & 284.507820 & 6.151308 & 4.593 & 6.730 & 6.740 & -0.010 & 0.020 & -0.030 & 0.061 & -0.010 \\
\hline HD 94924 & 292.748165 & -8.238385 & 4.349 & 8.130 & 8.010 & 0.120 & 0.122 & -0.002 & 0.372 & 0.830 \\
\hline HD 94985 & 285.137134 & 8.114793 & 9.124 & 6.061 & 5.898 & 0.163 & 0.006 & 0.157 & 0.018 & 0.680 \\
\hline HD 95109 & 289.057053 & 0.042811 & 0.479 & 7.210 & 6.110 & 1.100 & 0.400 & 0.700 & 1.267 & -6.755 \\
\hline HD 95752 & 291.393564 & -3.915900 & 0.314 & 7.421 & 6.970 & 0.451 & 0.447 & 0.004 & 1.364 & -6.909 \\
\hline HD 95993 & 291.028404 & -2.723612 & 2.260 & 8.620 & 8.180 & 0.440 & 0.245 & 0.195 & 0.755 & -0.804 \\
\hline HD 97082 & 290.085281 & 1.472661 & 0.796 & 7.590 & 6.790 & 0.800 & 0.164 & 0.636 & 0.518 & -4.224 \\
\hline HD 97152 & 290.946906 & -0.488398 & 0.371 & 8.030 & 8.070 & -0.040 & 0.352 & -0.392 & 1.051 & -5.133 \\
\hline HD 97485 & 291.472695 & -1.111831 & 0.704 & 8.830 & 7.900 & 0.930 & 0.236 & 0.694 & 0.747 & -3.610 \\
\hline HD 99757 & 289.751623 & 9.870446 & 2.160 & 8.060 & 8.160 & -0.100 & 0.036 & -0.136 & 0.109 & -0.277 \\
\hline HD 100148 & 292.067042 & 4.264463 & 0.326 & 8.900 & 8.190 & 0.710 & 0.208 & 0.502 & 0.652 & -4.894 \\
\hline HD 100213 & 294.807873 & -4.144356 & 0.422 & 9.520 & 9.340 & 0.180 & 0.499 & -0.319 & 1.495 & -4.027 \\
\hline HD 100359 & 297.394601 & -11.893218 & 1.867 & 7.081 & 6.884 & 0.197 & 0.323 & -0.126 & 0.978 & -2.739 \\
\hline HD 101947 & 295.179035 & -0.644405 & 0.588 & 5.830 & 5.030 & 0.800 & 0.253 & 0.547 & 0.795 & -6.917 \\
\hline HD 102541 & 290.030770 & 20.985574 & 8.604 & 8.180 & 7.940 & 0.240 & 0.006 & 0.234 & 0.019 & 2.595 \\
\hline HD 102682 & 291.846064 & 15.305595 & 3.918 & 8.789 & 8.240 & 0.549 & 0.052 & 0.497 & 0.163 & 1.042 \\
\hline HD 102893 & 295.568451 & 1.204261 & 1.120 & 8.280 & 8.250 & 0.030 & 0.147 & -0.117 & 0.445 & -1.949 \\
\hline HD 103285 & 296.026562 & 0.811795 & 1.347 & 8.310 & 8.240 & 0.070 & 0.138 & -0.068 & 0.419 & -1.533 \\
\hline HD 104036 & 300.068148 & -15.248520 & 9.566 & 6.918 & 6.738 & 0.180 & 0.009 & 0.171 & 0.028 & 1.614 \\
\hline HD 104631 & 297.305591 & 0.165498 & 0.464 & 6.808 & 6.771 & 0.037 & 0.372 & -0.335 & 1.114 & -6.010 \\
\hline HD 104705 & 297.454691 & -0.336299 & 0.432 & 9.560 & 9.110 & 0.450 & 0.386 & 0.064 & 1.181 & -3.891 \\
\hline HD 105509 & 294.957397 & 17.886055 & 10.916 & 5.991 & 5.746 & 0.245 & 0.006 & 0.239 & 0.019 & 0.918 \\
\hline HD 106111 & 299.636182 & -7.527775 & 1.131 & 7.010 & 6.170 & 0.840 & 0.227 & 0.613 & 0.716 & -4.277 \\
\hline HIP 107231 & 349.866388 & -48.057946 & 4.028 & 8.530 & 8.260 & 0.270 & 0.010 & 0.260 & 0.031 & 1.255 \\
\hline HD 107805 & 299.633990 & 1.064419 & 1.980 & 7.000 & 6.420 & 0.580 & 0.149 & 0.431 & 0.465 & -2.562 \\
\hline HD 108015 & 298.254125 & 15.478414 & 0.212 & 8.379 & 7.960 & 0.419 & 0.064 & 0.355 & 0.199 & -5.605 \\
\hline HD 108968 & 300.416306 & 3.351249 & 1.778 & 6.190 & 5.530 & 0.660 & 0.153 & 0.507 & 0.480 & -3.700 \\
\hline HD 110080 & 302.015990 & -7.694393 & 5.956 & 7.670 & 7.410 & 0.260 & 0.071 & 0.189 & 0.219 & 1.066 \\
\hline HD 110258 & 301.672146 & 3.053100 & 0.621 & 9.050 & 8.265 & 0.785 & 0.253 & 0.532 & 0.794 & -3.564 \\
\hline HD 110311 & 302.104209 & -6.550839 & 1.000 & 7.110 & 6.330 & 0.780 & 0.191 & 0.589 & 0.601 & -4.271 \\
\hline HD 111984 & 303.343188 & 19.794944 & 6.910 & 7.510 & 7.280 & 0.230 & 0.028 & 0.202 & 0.086 & 1.391 \\
\hline HD 112044 & 303.316382 & 4.438949 & 1.022 & 7.340 & 6.580 & 0.760 & 0.218 & 0.542 & 0.685 & -4.058 \\
\hline HD 112999 & 304.174646 & 2.175884 & 1.338 & 7.414 & 7.383 & 0.031 & 0.289 & -0.258 & 0.869 & -2.853 \\
\hline HD 114529 & 305.545614 & 2.845291 & 8.610 & 4.500 & 4.593 & -0.093 & 0.019 & -0.112 & 0.058 & -0.790 \\
\hline HD 115823 & 307.408458 & 9.870003 & 8.166 & 5.314 & 5.443 & -0.129 & 0.014 & -0.143 & 0.042 & -0.039 \\
\hline HD 116862 & 308.917726 & 13.072689 & 1.255 & 6.126 & 6.257 & -0.131 & 0.075 & -0.206 & 0.226 & -3.476 \\
\hline HD 117399 & 307.686744 & 0.918454 & 0.484 & 7.190 & 6.490 & 0.700 & 0.451 & 0.249 & 1.394 & -6.481 \\
\hline HD 118258 & 309.271499 & 6.154131 & 9.360 & 8.840 & 8.040 & 0.800 & 0.014 & 0.786 & 0.045 & 2.852 \\
\hline HD 118769 & 309.462365 & 4.637572 & 0.537 & 8.720 & 7.300 & 1.420 & 0.225 & 1.195 & 0.731 & -4.780 \\
\hline HD 119888 & 309.107132 & -1.669995 & 0.540 & 7.880 & 7.880 & 0.000 & 0.308 & -0.308 & 0.924 & -4.382 \\
\hline HD 120400 & 310.839533 & 4.377956 & 0.841 & 8.440 & 7.690 & 0.750 & 0.161 & 0.589 & 0.507 & -3.192 \\
\hline HD 121191 & 312.441366 & 8.159707 & 7.570 & 8.400 & 8.160 & 0.240 & 0.020 & 0.220 & 0.062 & 2.494 \\
\hline HD 121291 & 314.795173 & 16.737341 & 0.996 & 8.640 & 7.900 & 0.740 & 0.054 & 0.686 & 0.171 & -2.279 \\
\hline HD 122314 & 309.986969 & -4.857728 & 6.034 & 7.970 & 7.620 & 0.350 & 0.063 & 0.287 & 0.195 & 1.328 \\
\hline HD 122844 & 313.625108 & 6.651686 & 7.752 & 6.433 & 6.204 & 0.229 & 0.026 & 0.203 & 0.080 & 0.571 \\
\hline HD 123720 & 314.119582 & 5.685342 & 6.262 & 7.980 & 7.750 & 0.230 & 0.044 & 0.186 & 0.136 & 1.598 \\
\hline HD 124195 & 314.733924 & 6.351017 & 3.726 & 6.130 & 6.090 & 0.040 & 0.185 & -0.145 & 0.560 & -1.614 \\
\hline HD 124672 & 310.895742 & -6.521600 & 15.482 & 8.070 & 7.550 & 0.520 & 0.008 & 0.512 & 0.025 & 3.474 \\
\hline HD 124689 & 314.132939 & 3.145630 & 9.267 & 7.650 & 7.290 & 0.360 & 0.020 & 0.340 & 0.062 & 2.063 \\
\hline HD 125104 & 315.068011 & 4.908926 & 1.529 & 7.360 & 7.300 & 0.060 & 0.266 & -0.206 & 0.802 & -2.580 \\
\hline HD 125721 & 318.197805 & 11.829894 & 1.047 & 8.840 & 8.490 & 0.350 & 0.114 & 0.236 & 0.352 & -1.762 \\
\hline HD 125823 & 321.565642 & 20.022614 & 7.130 & 4.240 & 4.420 & -0.180 & 0.013 & -0.193 & 0.039 & -1.354 \\
\hline HD 126859 & 316.399573 & 4.142315 & 7.032 & 7.189 & 6.965 & 0.224 & 0.063 & 0.161 & 0.194 & 1.006 \\
\hline HD 127297 & 316.444594 & 3.307587 & 1.340 & 7.840 & 6.930 & 0.910 & 0.284 & 0.626 & 0.896 & -3.331 \\
\hline HD 127755 & 315.349490 & -0.303711 & 0.710 & 9.430 & 7.630 & 1.800 & 0.340 & 1.460 & 1.120 & -4.233 \\
\hline HD 127972 & 322.773998 & 16.669138 & 10.670 & 2.120 & 2.310 & -0.190 & 0.009 & -0.199 & 0.027 & -2.576 \\
\hline HD 128679 & 308.950206 & -16.205993 & 2.853 & 9.090 & 7.760 & 1.330 & 0.077 & 1.253 & 0.251 & -0.214 \\
\hline HD 129094 & 314.416788 & -4.710796 & 9.186 & 9.920 & 9.460 & 0.460 & 0.000 & 0.460 & 0.000 & 4.276 \\
\hline
\end{tabular}


Table 10

(Continued)

\begin{tabular}{|c|c|c|c|c|c|c|c|c|c|c|}
\hline $\begin{array}{r}\text { Name } \\
\ldots\end{array}$ & $\begin{array}{c}l \\
(\mathrm{deg})\end{array}$ & $\begin{array}{c}b \\
(\mathrm{deg})\end{array}$ & $\begin{array}{c}\varpi \\
\text { (mas) }\end{array}$ & $\begin{array}{c}B \\
(\mathrm{mag})\end{array}$ & $\begin{array}{c}V \\
(\mathrm{mag})\end{array}$ & $\begin{array}{c}(B-V) \\
(\mathrm{mag})\end{array}$ & $\begin{array}{c}E(B-V) \\
\quad(\mathrm{mag})\end{array}$ & $\begin{array}{l}(B-V)_{0} \\
\quad(\mathrm{mag})\end{array}$ & $\begin{array}{c}A_{V} \\
\text { (mag) }\end{array}$ & $\begin{array}{c}M_{V} \\
\text { (mag) }\end{array}$ \\
\hline HD 129118 & 323.658227 & 15.582768 & 6.354 & 7.780 & 6.790 & 0.990 & 0.014 & 0.976 & 0.045 & 0.760 \\
\hline HD 130233 & 313.973805 & -7.214961 & 1.016 & 8.290 & 7.440 & 0.850 & 0.180 & 0.670 & 0.569 & -3.095 \\
\hline HD 130701 & 315.825304 & -4.013205 & 1.745 & 6.660 & 5.960 & 0.700 & 0.133 & 0.567 & 0.418 & -3.249 \\
\hline HD 131638 & 325.032748 & 12.542091 & 2.462 & 8.330 & 8.320 & 0.010 & 0.062 & -0.052 & 0.189 & 0.088 \\
\hline HD 132247 & 323.127179 & 7.800860 & 4.932 & 8.270 & 8.090 & 0.180 & 0.052 & 0.128 & 0.160 & 1.396 \\
\hline HD 133880 & 329.181429 & 15.214997 & 9.652 & 5.650 & 5.790 & -0.140 & 0.011 & -0.151 & 0.033 & 0.680 \\
\hline HD 135240 & 319.688221 & -2.911210 & 1.556 & 5.030 & 5.090 & -0.060 & 0.152 & -0.212 & 0.458 & -4.408 \\
\hline HD 135411 & 332.251211 & 16.998319 & 1.070 & 9.900 & 8.220 & 1.680 & 0.097 & 1.583 & 0.321 & -1.954 \\
\hline HD 135592 & 316.976839 & -7.757915 & 1.475 & 6.960 & 6.390 & 0.570 & 0.116 & 0.454 & 0.363 & -3.128 \\
\hline HD 135876 & 330.846349 & 13.954524 & 6.974 & 5.495 & 5.604 & -0.109 & 0.012 & -0.121 & 0.036 & -0.215 \\
\hline HD 136633 & 319.961709 & -4.261537 & 1.143 & 8.280 & 8.230 & 0.050 & 0.171 & -0.121 & 0.518 & -1.998 \\
\hline HD 137164 & 319.624925 & -5.332615 & 8.763 & 9.180 & 8.140 & 1.040 & 0.019 & 1.021 & 0.061 & 2.792 \\
\hline HD 137518 & 329.807083 & 9.395976 & 0.582 & 7.830 & 7.750 & 0.080 & 0.178 & -0.098 & 0.540 & -3.964 \\
\hline HD 137626 & 318.422350 & -7.641804 & 0.889 & 8.610 & 7.800 & 0.810 & 0.141 & 0.669 & 0.446 & -2.902 \\
\hline HD 138521 & 317.798582 & -9.522212 & 3.742 & 8.050 & 8.040 & 0.010 & 0.062 & -0.052 & 0.189 & 0.717 \\
\hline HD 139534 & 319.148827 & -8.694991 & 4.067 & 8.810 & 7.810 & 1.000 & 0.065 & 0.935 & 0.208 & 0.648 \\
\hline HD 140566 & 332.392135 & 7.543717 & 3.791 & 8.510 & 8.290 & 0.220 & 0.064 & 0.156 & 0.197 & 0.987 \\
\hline HD 142049 & 323.988673 & 0.015545 & 19.510 & 6.210 & 5.850 & 0.360 & 0.005 & 0.355 & 0.016 & 2.286 \\
\hline HD 142542 & 342.704106 & 16.465330 & 18.766 & 6.718 & 6.284 & 0.434 & 0.003 & 0.431 & 0.009 & 2.642 \\
\hline HD 142941 & 322.129487 & -8.223853 & 1.075 & 7.190 & 6.410 & 0.780 & 0.092 & 0.688 & 0.291 & -3.724 \\
\hline HD 142994 & 338.396060 & 10.867654 & 6.347 & 7.460 & 7.170 & 0.290 & 0.011 & 0.279 & 0.034 & 1.149 \\
\hline HD 143028 & 321.769239 & -8.741536 & 0.706 & 7.720 & 7.800 & -0.080 & 0.105 & -0.185 & 0.317 & -3.273 \\
\hline HD 143098 & 340.463665 & 12.953532 & 30.331 & 8.330 & 7.640 & 0.690 & 0.002 & 0.688 & 0.006 & 5.043 \\
\hline HD 143232 & 338.403332 & 10.406861 & 6.784 & 6.890 & 6.660 & 0.230 & 0.008 & 0.222 & 0.025 & 0.793 \\
\hline HD 143999 & 323.231855 & -8.042597 & 0.877 & 8.430 & 7.890 & 0.540 & 0.111 & 0.429 & 0.346 & -2.741 \\
\hline HD 144951 & 328.159706 & -3.508135 & 0.940 & 8.080 & 8.070 & 0.010 & 0.251 & -0.241 & 0.755 & -2.821 \\
\hline HD 146323 & 327.753558 & -5.403478 & 1.062 & 7.490 & 6.490 & 1.000 & 0.192 & 0.808 & 0.611 & -3.992 \\
\hline HD 147170 & 333.339110 & -0.575511 & 4.619 & 8.895 & 8.263 & 0.632 & 0.101 & 0.531 & 0.317 & 1.269 \\
\hline HD 147683 & 344.856604 & 10.088824 & 3.387 & 7.160 & 7.050 & 0.110 & 0.355 & -0.245 & 1.068 & -1.369 \\
\hline HD 147894 & 335.606579 & 0.682539 & 6.090 & 7.241 & 7.312 & -0.071 & 0.081 & -0.152 & 0.245 & 0.990 \\
\hline HD 148891 & 315.735663 & -18.647736 & 3.946 & 8.050 & 8.000 & 0.050 & 0.044 & 0.006 & 0.134 & 0.846 \\
\hline HD 149238 & 324.027972 & -11.927103 & 18.618 & 8.580 & 8.040 & 0.540 & 0.006 & 0.534 & 0.019 & 4.371 \\
\hline HD 149404 & 340.537543 & 3.005780 & 0.760 & 5.880 & 5.520 & 0.360 & 0.442 & -0.082 & 1.342 & -6.419 \\
\hline HD 149450 & 338.788613 & 1.271181 & 1.121 & 8.229 & 8.239 & -0.010 & 0.262 & -0.272 & 0.787 & -2.300 \\
\hline HD 149455 & 331.781126 & -5.212324 & 4.271 & 7.710 & 7.690 & 0.020 & 0.149 & -0.129 & 0.451 & 0.391 \\
\hline HD 149668 & 327.191739 & -9.577443 & 2.115 & 7.710 & 7.610 & 0.100 & 0.079 & 0.021 & 0.241 & -1.004 \\
\hline HD 149715 & 330.389958 & -6.781829 & 3.569 & 9.420 & 8.330 & 1.090 & 0.135 & 0.955 & 0.433 & 0.659 \\
\hline HD 149779 & 339.877907 & 1.797286 & 1.154 & 7.740 & 7.560 & 0.180 & 0.306 & -0.126 & 0.927 & -3.056 \\
\hline HD 151158 & 341.876698 & 1.450784 & 0.856 & 8.480 & 8.250 & 0.230 & 0.419 & -0.189 & 1.265 & -3.353 \\
\hline HD 151475 & 338.875985 & -1.588860 & 0.992 & 8.170 & 8.060 & 0.110 & 0.266 & -0.156 & 0.804 & -2.762 \\
\hline HD 151564 & 343.130554 & 1.910493 & 0.737 & 8.100 & 7.980 & 0.120 & 0.438 & -0.318 & 1.313 & -3.995 \\
\hline HD 151665 & 315.626975 & -20.092090 & 2.907 & 8.320 & 8.070 & 0.250 & 0.056 & 0.194 & 0.173 & 0.215 \\
\hline HD 151890 & 346.115149 & 3.913990 & 3.726 & 2.820 & 2.980 & -0.160 & 0.181 & -0.341 & 0.542 & -4.705 \\
\hline HD 152333 & 343.827246 & 1.374939 & 0.735 & 8.930 & 8.840 & 0.090 & 0.434 & -0.344 & 1.299 & -3.128 \\
\hline HD 152478 & 336.782978 & -4.635986 & 3.222 & 6.310 & 6.330 & -0.020 & 0.244 & -0.264 & 0.733 & -1.863 \\
\hline HD 152511 & 328.996568 & -10.888160 & 4.900 & 6.468 & 6.530 & -0.062 & 0.058 & -0.120 & 0.176 & -0.195 \\
\hline HD 152667 & 344.531027 & 1.457096 & 0.607 & 6.510 & 6.220 & 0.290 & 0.436 & -0.146 & 1.319 & -6.183 \\
\hline HD 152901 & 346.897945 & 3.025074 & 3.115 & 7.450 & 7.390 & 0.060 & 0.187 & -0.127 & 0.566 & -0.709 \\
\hline HD 153004 & 350.412633 & 5.666730 & 2.270 & 7.370 & 6.610 & 0.760 & 0.272 & 0.488 & 0.852 & -2.462 \\
\hline HD 153140 & 340.596082 & -2.424087 & 0.926 & 7.850 & 7.500 & 0.350 & 0.327 & 0.023 & 0.999 & -3.665 \\
\hline HD 153747 & 347.139655 & 1.962184 & 5.469 & 7.540 & 7.420 & 0.120 & 0.033 & 0.087 & 0.101 & 1.008 \\
\hline HD 153919 & 347.754424 & 2.173487 & 0.549 & 6.780 & 6.510 & 0.270 & 0.402 & -0.132 & 1.217 & -6.011 \\
\hline HD 154339 & 340.795488 & -3.813101 & 0.837 & 9.740 & 9.190 & 0.550 & 0.345 & 0.205 & 1.064 & -2.259 \\
\hline HD 155190 & 328.390770 & -13.744251 & 4.083 & 7.100 & 7.130 & -0.030 & 0.060 & -0.090 & 0.182 & 0.003 \\
\hline HD 155550 & 352.960668 & 3.540731 & 0.641 & 8.130 & 8.070 & 0.060 & 0.328 & -0.268 & 0.986 & -3.881 \\
\hline HD 155555 & 324.898586 & -16.297357 & 32.780 & 7.499 & 6.723 & 0.776 & 0.003 & 0.773 & 0.010 & 4.292 \\
\hline HD 155775 & 348.796715 & 0.145558 & 0.877 & 8.660 & 8.610 & 0.050 & 0.306 & -0.256 & 0.920 & -2.595 \\
\hline HD 155781 & 330.235394 & 0.180648 & 3.986 & 7.550 & 7.420 & 0.130 & 0.059 & 0.071 & 0.181 & 0.242 \\
\hline HD 156408 & 349.316308 & -0.389699 & 3.378 & 8.650 & 8.270 & 0.380 & 0.192 & 0.188 & 0.592 & 0.322 \\
\hline HD 156623 & 343.479370 & -4.832280 & 8.948 & 7.350 & 7.260 & 0.090 & 0.008 & 0.082 & 0.025 & 1.994 \\
\hline HD 156768 & 333.053617 & -12.071444 & 2.980 & 7.016 & 5.872 & 1.144 & 0.079 & 1.065 & 0.255 & -2.012 \\
\hline HD 156853 & 340.014519 & -7.486891 & 2.747 & 7.560 & 7.600 & -0.040 & 0.116 & -0.156 & 0.351 & -0.557 \\
\hline HD 156979 & 343.510397 & -5.220728 & 0.977 & 7.670 & 6.740 & 0.930 & 0.245 & 0.685 & 0.775 & -4.086 \\
\hline
\end{tabular}


Table 10

(Continued)

\begin{tabular}{|c|c|c|c|c|c|c|c|c|c|c|}
\hline $\begin{array}{r}\text { Name } \\
\ldots\end{array}$ & $\begin{array}{c}l \\
(\mathrm{deg})\end{array}$ & $\begin{array}{c}b \\
(\mathrm{deg})\end{array}$ & $\begin{array}{c}\varpi \\
\text { (mas) }\end{array}$ & $\begin{array}{c}B \\
(\mathrm{mag})\end{array}$ & $\begin{array}{c}V \\
(\mathrm{mag})\end{array}$ & $\begin{array}{c}(B-V) \\
(\mathrm{mag})\end{array}$ & $\begin{array}{c}E(B-V) \\
\quad(\mathrm{mag})\end{array}$ & $\begin{array}{l}(B-V)_{0} \\
\quad(\mathrm{mag})\end{array}$ & $\begin{array}{c}A_{V} \\
\text { (mag) }\end{array}$ & $\begin{array}{c}M_{V} \\
\text { (mag) }\end{array}$ \\
\hline HD 157321 & 327.178343 & -16.158987 & 3.939 & 8.350 & 8.020 & 0.330 & 0.051 & 0.279 & 0.158 & 0.839 \\
\hline HD 158155 & 354.660584 & 0.814982 & 0.712 & 8.720 & 8.330 & 0.390 & 0.389 & 0.001 & 1.187 & -3.594 \\
\hline HD 158186 & 355.906751 & 1.596463 & 0.940 & 7.070 & 7.040 & 0.030 & 0.268 & -0.238 & 0.807 & -3.902 \\
\hline HD 158443 & 354.359038 & 0.173160 & 0.920 & 8.780 & 7.910 & 0.870 & 0.298 & 0.572 & 0.937 & -3.207 \\
\hline HD 159041 & 342.732780 & -8.188957 & 1.634 & 7.990 & 8.040 & -0.050 & 0.079 & -0.129 & 0.239 & -1.133 \\
\hline HD 159441 & 335.178910 & -13.204213 & 9.550 & 7.690 & 7.360 & 0.330 & 0.008 & 0.322 & 0.025 & 2.235 \\
\hline HD 159654 & 349.029646 & -4.865982 & 0.840 & 7.990 & 7.260 & 0.730 & 0.199 & 0.531 & 0.625 & -3.744 \\
\hline HD 160589 & 356.568493 & -1.268251 & 5.326 & 8.180 & 7.850 & 0.330 & 0.051 & 0.279 & 0.158 & 1.324 \\
\hline HD 161592 & 1.166282 & 0.209286 & 3.431 & 5.340 & 4.540 & 0.800 & 0.173 & 0.627 & 0.546 & -3.328 \\
\hline HD 161783 & 338.940199 & -13.195838 & 1.973 & 5.600 & 5.710 & -0.110 & 0.122 & -0.232 & 0.367 & -3.182 \\
\hline HD 162102 & 356.489248 & -3.419668 & 0.798 & 8.790 & 7.510 & 1.280 & 0.280 & 1.000 & 0.901 & -3.882 \\
\hline HD 163181 & 358.125130 & -3.774663 & 0.511 & 6.990 & 6.610 & 0.380 & 0.372 & 0.008 & 1.135 & -5.984 \\
\hline HD 163254 & 349.894016 & -8.614876 & 1.730 & 6.632 & 6.728 & -0.096 & 0.083 & -0.179 & 0.251 & -2.333 \\
\hline HD 163482 & 355.219541 & -5.828797 & 7.071 & 6.870 & 6.830 & 0.040 & 0.024 & 0.016 & 0.073 & 1.004 \\
\hline HD 163708 & 354.530336 & -6.509347 & 5.712 & 7.155 & 7.089 & 0.066 & 0.033 & 0.033 & 0.101 & 0.772 \\
\hline HD 164975 & 1.575794 & -3.979580 & 1.180 & 5.470 & 4.690 & 0.780 & 0.119 & 0.661 & 0.376 & -5.327 \\
\hline HD 166596 & 351.887336 & -10.965792 & 1.643 & 5.298 & 5.462 & -0.164 & 0.053 & -0.217 & 0.160 & -3.619 \\
\hline HD 167231 & 357.282255 & -8.844732 & 3.292 & 7.520 & 7.420 & 0.100 & 0.050 & 0.050 & 0.153 & -0.145 \\
\hline HD 167714 & 313.739664 & -25.774726 & 9.226 & 7.133 & 5.946 & 1.187 & 0.007 & 1.180 & 0.023 & 0.748 \\
\hline HD 168403 & 354.693942 & -11.317882 & 4.582 & 6.900 & 6.790 & 0.110 & 0.027 & 0.083 & 0.083 & 0.012 \\
\hline HD 168651 & 346.130512 & -15.563721 & 9.614 & 7.700 & 7.400 & 0.300 & 0.008 & 0.292 & 0.025 & 2.290 \\
\hline HD 168740 & 331.834147 & -21.150130 & 14.147 & 6.313 & 6.122 & 0.191 & 0.006 & 0.185 & 0.018 & 1.857 \\
\hline HD 170461 & 357.594735 & -12.142131 & 10.493 & 7.280 & 6.980 & 0.300 & 0.006 & 0.294 & 0.019 & 2.066 \\
\hline HD 171577 & 352.316173 & -15.758740 & 3.472 & 7.780 & 7.750 & 0.030 & 0.037 & -0.007 & 0.113 & 0.340 \\
\hline HD 171819 & 347.618520 & -17.856894 & 10.163 & 6.058 & 5.840 & 0.218 & 0.008 & 0.210 & 0.025 & 0.850 \\
\hline HD 172416 & 347.960987 & -18.296431 & 12.444 & 7.080 & 6.620 & 0.460 & 0.007 & 0.453 & 0.022 & 2.073 \\
\hline HD 172995 & 347.659912 & -18.979164 & 6.290 & 7.010 & 6.810 & 0.200 & 0.018 & 0.182 & 0.055 & 0.748 \\
\hline HD 173344 & 332.368984 & -23.771926 & 6.866 & 7.590 & 7.410 & 0.180 & 0.014 & 0.166 & 0.043 & 1.551 \\
\hline HD 173794 & 344.012056 & -20.878022 & 6.220 & 7.350 & 7.120 & 0.230 & 0.020 & 0.210 & 0.062 & 1.027 \\
\hline HD 174139 & 338.884357 & -22.668354 & 3.165 & 8.240 & 8.170 & 0.070 & 0.047 & 0.023 & 0.144 & 0.528 \\
\hline HD 174632 & 5.157964 & -13.722970 & 4.227 & 6.607 & 6.638 & -0.031 & 0.056 & -0.087 & 0.170 & -0.402 \\
\hline HD 174694 & 328.286503 & -25.387694 & 5.199 & 5.080 & 4.400 & 0.680 & 0.016 & 0.664 & 0.051 & -2.071 \\
\hline HD 175008 & 323.165007 & -26.345258 & 5.397 & 6.750 & 6.790 & -0.040 & 0.011 & -0.051 & 0.033 & 0.417 \\
\hline HD 177171 & 344.540829 & -23.346929 & 16.990 & 5.700 & 5.174 & 0.526 & 0.006 & 0.520 & 0.019 & 1.306 \\
\hline HD 177523 & 349.613583 & -22.216656 & 7.289 & 7.780 & 7.490 & 0.290 & 0.012 & 0.278 & 0.037 & 1.766 \\
\hline HD 177665 & 351.911604 & -21.656600 & 5.185 & 8.690 & 8.360 & 0.330 & 0.025 & 0.305 & 0.078 & 1.856 \\
\hline HD 177776 & 340.946298 & -24.572862 & 3.143 & 8.150 & 8.120 & 0.030 & 0.038 & -0.008 & 0.116 & 0.491 \\
\hline HD 179522 & 349.644068 & -23.582311 & 8.066 & 8.330 & 7.430 & 0.900 & 0.010 & 0.890 & 0.032 & 1.931 \\
\hline HD 184035 & 358.915878 & -24.806037 & 6.696 & 6.000 & 5.910 & 0.090 & 0.019 & 0.071 & 0.058 & -0.019 \\
\hline HD 185139 & 353.528055 & -27.057738 & 9.902 & 6.530 & 6.260 & 0.270 & 0.007 & 0.263 & 0.022 & 1.217 \\
\hline HD 187418 & 352.217174 & -29.447853 & 3.583 & 8.590 & 8.310 & 0.280 & 0.019 & 0.261 & 0.059 & 1.023 \\
\hline HD 189631 & 358.788751 & -30.384035 & 11.087 & 7.840 & 7.540 & 0.300 & 0.006 & 0.294 & 0.019 & 2.746 \\
\hline HD 189951 & 354.800696 & -31.301714 & 5.250 & 8.100 & 7.830 & 0.270 & 0.012 & 0.258 & 0.037 & 1.394 \\
\hline HD 191585 & 331.765945 & -33.205593 & 5.120 & 7.060 & 6.920 & 0.140 & 0.014 & 0.126 & 0.043 & 0.423 \\
\hline HD 192316 & 314.682531 & -30.934019 & 7.588 & 7.770 & 7.550 & 0.220 & 0.007 & 0.213 & 0.022 & 1.929 \\
\hline HD 192594 & 2.167096 & -32.659315 & 3.073 & 8.700 & 7.340 & 1.360 & 0.025 & 1.335 & 0.082 & -0.304 \\
\hline HD 193174 & 9.694296 & -31.884618 & 7.106 & 7.500 & 7.250 & 0.250 & 0.013 & 0.237 & 0.040 & 1.468 \\
\hline HD 193677 & 343.593132 & -35.073332 & 8.396 & 7.760 & 7.600 & 0.160 & 0.008 & 0.152 & 0.025 & 2.196 \\
\hline HD 198592 & 353.432167 & -39.866271 & 6.508 & 7.780 & 7.580 & 0.200 & 0.008 & 0.192 & 0.025 & 1.623 \\
\hline HD 198736 & 329.508381 & -37.301504 & 5.965 & 8.630 & 8.340 & 0.290 & 0.008 & 0.282 & 0.025 & 2.193 \\
\hline HD 198752 & 6.960965 & -39.376813 & 3.287 & 8.620 & 7.140 & 1.480 & 0.018 & 1.462 & 0.059 & -0.335 \\
\hline HD 200203 & 337.197874 & -39.987120 & 5.505 & 7.530 & 7.350 & 0.180 & 0.009 & 0.171 & 0.028 & 1.026 \\
\hline HD 200475 & 319.771576 & -35.486409 & 4.474 & 8.080 & 7.820 & 0.260 & 0.021 & 0.239 & 0.065 & 1.008 \\
\hline HD 200670 & 7.258495 & -41.851515 & 12.481 & 8.340 & 7.810 & 0.530 & 0.004 & 0.526 & 0.013 & 3.279 \\
\hline HD 201247 & 342.588946 & -41.727507 & 28.760 & 7.700 & 7.100 & 0.600 & 0.004 & 0.596 & 0.013 & 4.381 \\
\hline HD 201292 & 310.233667 & -31.637037 & 4.720 & 8.510 & 8.190 & 0.320 & 0.094 & 0.226 & 0.290 & 1.270 \\
\hline HD 201427 & 345.509006 & -42.280150 & 20.650 & 7.730 & 7.068 & 0.662 & 0.005 & 0.657 & 0.016 & 3.627 \\
\hline HD 203244 & 324.896406 & -38.909688 & 48.062 & 7.700 & 6.970 & 0.730 & 0.001 & 0.729 & 0.003 & 5.376 \\
\hline HD 204352 & 339.381016 & -43.994561 & 5.251 & 8.660 & 8.400 & 0.260 & 0.008 & 0.252 & 0.025 & 1.977 \\
\hline HD 204370 & 347.479373 & -45.467633 & 4.886 & 7.820 & 7.520 & 0.300 & 0.009 & 0.291 & 0.028 & 0.937 \\
\hline HD 205834 & 307.420813 & -30.917878 & 4.261 & 9.340 & 8.110 & 1.230 & 0.093 & 1.137 & 0.301 & 0.956 \\
\hline HD 205877 & 344.098347 & -46.530732 & 5.135 & 6.784 & 6.202 & 0.582 & 0.008 & 0.574 & 0.025 & -0.271 \\
\hline HD 208094 & 305.516566 & -29.818144 & 4.135 & 8.440 & 8.210 & 0.230 & 0.081 & 0.149 & 0.249 & 1.043 \\
\hline
\end{tabular}


Table 10

(Continued)

\begin{tabular}{|c|c|c|c|c|c|c|c|c|c|c|}
\hline $\begin{array}{r}\text { Name } \\
\ldots\end{array}$ & $\begin{array}{c}l \\
(\mathrm{deg})\end{array}$ & $\begin{array}{c}b \\
(\mathrm{deg})\end{array}$ & $\begin{array}{c}\varpi \\
(\mathrm{mas})\end{array}$ & $\begin{array}{c}B \\
(\mathrm{mag})\end{array}$ & $\begin{array}{c}V \\
(\mathrm{mag})\end{array}$ & $\begin{array}{c}(B-V) \\
(\mathrm{mag})\end{array}$ & $\begin{array}{c}E(B-V) \\
\quad(\mathrm{mag})\end{array}$ & $\begin{array}{c}(B-V)_{0} \\
(\mathrm{mag})\end{array}$ & $\begin{array}{c}A_{V} \\
(\mathrm{mag})\end{array}$ & $\begin{array}{c}M_{V} \\
\text { (mag) }\end{array}$ \\
\hline HD 208614 & 352.610492 & -51.199348 & 3.686 & 7.870 & 7.720 & 0.150 & 0.009 & 0.141 & 0.028 & 0.525 \\
\hline HD 209234 & 331.450421 & -46.325930 & 23.352 & 8.480 & 7.870 & 0.610 & 0.003 & 0.607 & 0.009 & 4.702 \\
\hline HD 210572 & 338.681592 & -50.347903 & 13.575 & 8.238 & 7.714 & 0.524 & 0.004 & 0.520 & 0.013 & 3.365 \\
\hline HD 212661 & 332.056482 & -50.187132 & 6.910 & 7.080 & 6.910 & 0.170 & 0.005 & 0.165 & 0.015 & 1.092 \\
\hline HD 213669 & 336.103568 & -53.075908 & 8.779 & 7.620 & 7.420 & 0.200 & 0.004 & 0.196 & 0.012 & 2.125 \\
\hline HD 216668 & 317.189250 & -45.028130 & 3.871 & 7.980 & 7.880 & 0.100 & 0.015 & 0.085 & 0.046 & 0.773 \\
\hline HD 216743 & 352.070787 & -61.942147 & 9.768 & 7.413 & 7.247 & 0.166 & 0.003 & 0.163 & 0.009 & 2.187 \\
\hline HD 217522 & 346.722689 & -61.851320 & 9.715 & 7.990 & 7.520 & 0.470 & 0.003 & 0.467 & 0.009 & 2.448 \\
\hline HD 218090 & 325.729448 & -53.525332 & 5.474 & 8.420 & 8.130 & 0.290 & 0.006 & 0.284 & 0.019 & 1.803 \\
\hline HD 219301 & 326.385409 & -55.844349 & 10.907 & 6.840 & 6.560 & 0.280 & 0.003 & 0.277 & 0.009 & 1.739 \\
\hline HD 220633 & 345.169775 & -66.525355 & 2.200 & 8.770 & 8.290 & 0.480 & 0.009 & 0.471 & 0.028 & -0.026 \\
\hline
\end{tabular}

\section{ORCID iDs}

Samuel N. Mellon (1) https://orcid.org/0000-0003-3405-2864 Eric E. Mamajek (1) https://orcid.org/0000-0003-2008-1488 Remko Stuik (i) https://orcid.org/0000-0001-7797-3749 Konstanze Zwintz (D) https://orcid.org/0000-0001-9229-8315 Matthew A. Kenworthy (iD https://orcid.org/0000-00027064-8270

Geert Jan J. Talens (iD https://orcid.org/0000-0003-4787-2335 Olivier Burggraaff (i) https://orcid.org/0000-0002-2487-4533 John I. Bailey, III (i) https://orcid.org/0000-0002-4272-263X Patrick Dorval (i) https://orcid.org/0000-0003-3812-2436 Blaine B. D. Lomberg (ib https://orcid.org/0000-00021520-7851

Rudi B. Kuhn (iD https://orcid.org/0000-0002-4236-9020 Michael J. Ireland (iD https://orcid.org/0000-0002-6194-043X

\section{References}

Blažko, S. 1907, AN, 175, 325

Boro Saikia, S., Marvin, C. J., Jeffers, S. V., et al. 2018, A\&A, 616, A108 Borucki, W. J., Koch, D., Basri, G., et al. 2010, Sci, 327, 977 Bos, M. 1994, ExA, 5, 13

Boss, B. 1937, General Catalogue of 33342 Stars for the Epoch 1950, Vol. 1 and 5 (Washington, DC: Carnegie Instit. Washington)

Bowman, D. M., \& Kurtz, D. W. 2018, MNRAS, 476, 3169

Breger, M., \& Pamyatnykh, A. A. 1998, A\&A, 332, 958

Bressan, A., Marigo, P., Girardi, L., et al. 2012, MNRAS, 427, 127

Brown, A. G. A., Vallenari, A., Prusti, T., et al. 2018, A\&A, 616, A1

Burggraaff, O., Talens, G. J. J., Spronck, J., et al. 2018, A\&A, 617, A32

Buscombe, W. 1969, MNRAS, 144, 31

Capitanio, L., Lallement, R., Vergely, J. L., Elyajouri, M., \& Monreal-Ibero, A. 2017, A\&A, 606, A65

Cargile, P. A., James, D. J., Pepper, J., et al. 2014, ApJ, 782, 29

Collins, K. A., Collins, K. I., Pepper, J., et al. 2018, AJ, 156, 234

Corbally, C. J. 1984, ApJS, 55, 657

Cousins, A. W. J., \& Lagerweij, H. C. 1971, MNSSA, 30, 12

Cucchiaro, A., Macau-Hercot, D., Jaschek, M., \& Jaschek, C. 1977, A\&AS, 30,71

da Silva, R., Maceroni, C., Gandolfi, D., Lehmann, H., \& Hatzes, A. P. 2014, A\&A, 565, A55

De Cat, P. 2007, CoAst, 150, 167

De Mey, K., Daems, K., \& Sterken, C. 1998, A\&A, 336, 527

Dorval, P., Talens, G. J. J., Otten, G. P. P. L., et al. 2019, arXiv:1904.02733

Gallet, F., \& Bouvier, J. 2013, A\&A, 556, A36

Garrison, R. F., Hiltner, W. A., \& Schild, R. E. 1977, ApJS, 35, 111
Gray, R. O., Corbally, C. J., Garrison, R. F., et al. 2006, AJ, 132, 161

Gray, R. O., \& Garrison, R. F. 1987, ApJS, 65, 581

Gray, R. O., \& Garrison, R. F. 1989, ApJS, 70, 623

Gray, R. O., Riggs, Q. S., Koen, C., et al. 2017, AJ, 154, 31

Groenewegen, M. A. T. 2018, A\&A, 619, A8

Hartman, J. D., Bakos, G. Á., Kovács, G., \& Noyes, R. W. 2010, MNRAS, 408,475

Hiltner, W. A., Garrison, R. F., \& Schild, R. E. 1969, ApJ, 157, 313

Houk, N. 1978, Michigan Catalogue of Two-dimensional Spectral Types for the HD Stars, Vol. 2 (Ann Harbor, MI: Michigan Univ. Press)

Houk, N. 1982, Michigan Catalogue of Two-dimensional Spectral Types for the HD Stars, Vol. 3 (Ann Arbor, MI: Michigan Univ. Press)

Houk, N., \& Cowley, A. P. 1975, University of Michigan Catalogue of Twodimensional Spectral Types for the HD Stars, Vol. I

Houk, N., Swift, C. M., Murray, C. A., Penston, M. J., \& Binney, J. J. 1997, ESA Spec. Publ., 402, 279

Howell, S. B., Sobeck, C., Haas, M., et al. 2014, PASP, 126, 398

Hube, D. P. 1970, MmRAS, 72, 233

Hunter, J. D. 2007, Matplotlib: A 2D Graphics Environment, http://www. scipy.org/

Jones, E., Oliphant, T., Peterson, P., et al. 2001, SciPy: Open Source Scientific Tools for Python, http://www.scipy.org/

Kalas, P., Jinfei Wang, J., Wang, L., et al. 2019, AAS Meeting, 233, 218.03

Keenan, P. C., \& McNeil, R. C. 1989, ApJS, 71, 245

Kenworthy, M. A., \& Mamajek, E. E. 2015, ApJ, 800, 126

Kharchenko, N. V. 2001, KFNT, 17, 409

Kharchenko, N. V., \& Roeser, S. 2009, yCat, 1280

Koen, C., \& Eyer, L. 2002, MNRAS, 331, 45

Kurtz, D. W. 1980, MNRAS, 193, 61

Lallement, R., Capitanio, L., Ruiz-Dern, L., et al. 2018, A\&A, 616, A132

Lefèvre, L., Marchenko, S. V., Moffat, A. F. J., \& Acker, A. 2009, A\&A, 507,1141

Levato, H. 1975, A\&AS, 19, 91

Levato, H., Malaroda, S., Morrell, N., Solivella, G., \& Grosso, M. 1996, A\&AS, 118, 231

Levenhagen, R. S., \& Leister, N. V. 2006, MNRAS, 371, 252

Liakos, A., \& Niarchos, P. 2017, MNRAS, 465, 1181

Luckas, P. 2018, ATel, 11460, 1

Mamajek, E. E., \& Hillenbrand, L. A. 2008, ApJ, 687, 1264

Mamajek, E. E., Quillen, A. C., Pecaut, M. J., et al. 2012, AJ, 143, 72

Marigo, P., Girardi, L., Bressan, A., et al. 2017, ApJ, 835, 77

Mason, B. D., Wycoff, G. L., Hartkopf, W. I., Douglass, G. G., \& Worley, C. E. 2019, yCat, 102026

Mékarnia, D., Chapellier, E., Guillot, T., et al. 2017, A\&A, 608, 6

Mellon, S. N., Mamajek, E. E., Oberst, T. E., \& Pecaut, M. J. 2017, ApJ, 844, 66

Mellon, S. N., Mamajek, E. E., Zwintz, K., et al. 2019a, ApJ, 870, 36

Mellon, S. N., Stuik, R., Kenworthy, M., et al. 2019b, AAS Meeting Abstracts, $233,140.22$ 
Mentel, R. T., Kenworthy, M. A., Cameron, D. A., et al. 2018, A\&A, 619, A157

Miglio, A., Montalbán, J., \& Dupret, M.-A. 2007, CoAst, 151, 48

Moe, M., \& Kratter, K. M. 2018, ApJ, 854, 44

Montgomery, M. H., \& Odonoghue, D. 1999, DSSN, 13, 28

Nagy, T. A. 1985 , BAAS, 17,887

Oberst, T. E., Rodriguez, J. E., Colón, K. D., et al. 2017, AJ, 153, 97

Ochsenbein, F., Bauer, P., \& Marcout, J. 2000, A\&AS, 143, 23

O'Connell, D. J. K. 1951, PRCO, 2, 85

Osborn, J., Föhring, D., Dhillon, V. S., \& Wilson, R. W. 2015, MNRAS, 452, 1707

Paunzen, E., \& Duffee, B. 1996, IBVS, 4297, 1

Paunzen, E., Duffee, B., Heiter, U., Kuschnig, R., \& Weiss, W. W. 2001, A\&A, 373, 625

Paunzen, E., Weiss, W. W., Kuschnig, R., et al. 1998, A\&A, 335, 533

Pepper, J., Pogge, R. W., DePoy, D. L., et al. 2007, PASP, 119, 923

Perryman, M. A. C. 1997, ESA Spec. Publ., 1200

Pojmanski, G. 2002, AcA, 52, 397

Press, W. H., Teukolsky, S. A., Vetterling, W. T., \& Flannery, B. P. 1992, Numerical Recipes in FORTRAN. The Art of Scientific Computing (Cambridge: Cambridge Univ. Press)

Pribulla, T., Kreiner, J. M., \& Tremko, J. 2003, CoSka, 33, 38

Pribulla, T., Vaňko, M., Chochol, D., Hambálek, L., \& Parimucha, Š. 2011, AN, 332, 607

Rabus, M., \& Prieto, J. L. 2018, ATel, 11506, 1

Rimoldini, L., Dubath, P., Süveges, M., et al. 2012, MNRAS, 427, 2917

Rodríguez, E., López-González, M. J., \& López de Coca, P. 2000, A\&AS, 144,469

Rossum, G. 1995, Python Reference Manual, CWI Rep. CS-R9525, (Amsterdam: Centrum voor Wiskunde en Informatica)

Sahade, J. 1952, ApJ, 116, 35
Scargle, J. D. 1982, ApJ, 263, 835

Shappee, B. J., Prieto, J. L., Grupe, D., et al. 2014, ApJ, 788, 48

Shara, M. M., Moffat, A. F. J., Gerke, J., et al. 2009, AJ, 138, 402

Sitek, M., \& Pojmański, G. 2014, AcA, 64, 115

Soderblom, D. R. 1990, AJ, 100, 204

Sota, A., Maíz Apellániz, J., Morrell, N. I., et al. 2014, ApJS, 211, 10

Stanek, K. Z., Holoien, T. W. S., Kochanek, C. S., et al. 2018, ATel, 11454, 1 Stéfan van der Walt, S. C. C., \& Varoquaux, G. 2011, The NumPy Array: A

Structure for Efficient Numerical Computation, http://www.scipy.org/ Sterken, C. 1997, A\&A, 325, 563

Stobie, R. S., \& Shobbrook, R. R. 1976, MNRAS, 174, 401

Strader, J., Chomiuk, L., Holoien, T. W. S., et al. 2018, ATel, 11456, 1

Stuik, R., Bailey, J. I., Dorval, P., et al. 2017, A\&A, 607, A45

Talens, G. J. J., Albrecht, S., Spronck, J. F. P., et al. 2017a, A\&A, 606, A73

Talens, G. J. J., Deul, E. R., Stuik, R., et al. 2018, A\&A, 619, A154

Talens, G. J. J., Spronck, J. F. P., Lesage, A.-L., et al. 2017b, A\&A, 601, A11

Terrell, D., Munari, U., Zwitter, T., \& Nelson, R. H. 2003, AJ, 126, 2988

The Astropy Collaboration, Price-Whelan, A. M., Sipőcz, B. M., et al. 2018, AJ, 156, 123

Torres, C. A. O., Quast, G. R., da Silva, L., et al. 2006, A\&A, 460, 695

Udalski, A., Szymanski, M. K., Soszynski, I., \& Poleski, R. 2008, AcA, 58, 69 van Leeuwen, F. 2007, A\&A, 474, 653

van Werkhoven, T. I. M., Kenworthy, M. A., \& Mamajek, E. E. 2014, MNRAS, 441, 2845

Watson, C. L., Henden, A. A., \& Price, A. 2006, SASS, 25, 47

Wenger, M., Ochsenbein, F., Egret, D., et al. 2000, A\&AS, 143, 9

Wilsey, N. J., \& Beaky, M. M. 2009, SASS, 28, 107

Young, A. T. 1967, AJ, 72, 747

Zwintz, K., Fossati, L., Ryabchikova, T., et al. 2014a, Sci, 345, 550

Zwintz, K., Reese, D. R., Neiner, C., et al. 2019, A\&A, 627, A28

Zwintz, K., Ryabchikova, T., Lenz, P., et al. 2014b, A\&A, 567, A4 